\title{
Supergeometry in locally covariant quantum field theory
}

\author{
Thomas-Paul Hack ${ }^{1,2, a}$, Florian Hanisch ${ }^{3, b}$ and Alexander Schenkel ${ }^{4, c}$ \\ ${ }^{1}$ Dipartimento di Matematica, Università degli Studi di Genova, \\ Via Dodecaneso 35, 16146 Genova, Italy. \\ 2 Institut für Theoretische Physik, Universität Leipzig, \\ Brüderstraße 16, 04103 Leipzig, Germany. \\ ${ }^{3}$ Institut für Mathematik, Universität Potsdam, \\ Karl-Liebknecht-Straße 24-25, 14476 Golm (Potsdam), Germany. \\ ${ }^{4}$ Department of Mathematics, Heriot-Watt University, \\ Colin Maclaurin Building, Riccarton, Edinburgh EH14 4AS, United Kingdom. \\ Email: ${ }^{a}$ hack@dima.unige.it,${ }^{b}$ fhanisch@math.uni-potsdam.de , ${ }^{c}$ as880@hw.ac.uk
}

September 2015

\begin{abstract}
In this paper we analyze supergeometric locally covariant quantum field theories. We develop suitable categories SLoc of super-Cartan supermanifolds, which generalize Lorentz manifolds in ordinary quantum field theory, and show that, starting from a few representation theoretic and geometric data, one can construct a functor $\mathfrak{A}:$ SLoc $\rightarrow \mathrm{S}^{*}$ Alg to the category of super- $*$-algebras which can be interpreted as a non-interacting super-quantum field theory. This construction turns out to disregard supersymmetry transformations as the morphism sets in the above categories are too small. We then solve this problem by using techniques from enriched category theory, which allows us to replace the morphism sets by suitable morphism supersets that contain supersymmetry transformations as their higher superpoints. We construct super-quantum field theories in terms of enriched functors $\mathfrak{e A}: \mathrm{eSLoc} \rightarrow \mathrm{eS}^{*} \mathrm{Alg}$ between the enriched categories and show that supersymmetry transformations are appropriately described within the enriched framework. As examples we analyze the superparticle in 1|1-dimensions and the free Wess-Zumino model in $3 \mid 2$ dimensions.
\end{abstract}

Report no.: EMPG-15-01

Keywords: supergeometry, algebraic quantum field theory, locally covariant quantum field theory, enriched category theory

MSC 2010: 81T05, 58A50, 81T60, 83E50 


\section{Contents}

1 Introduction and summary 2

2 Preliminaries on supergeometry 5

3 Super-Cartan supermanifolds 12

3.1 Representation theoretic data and super-Poincaré super-Lie algebras . . . . . . 12

3.2 Super-Cartan structures . . . . . . . . . . . . . . . . . . . . . 14

3.3 Lorentz geometry on the reduced manifold . . . . . . . . . . . . . . . . . 15

3.4 The category of globally hyperbolic super-Cartan supermanifolds . . . . . . . 16

4 Axiomatic definition of super-field theories 16

5 Construction of super-quantum field theories 18

5.1 The functor $\mathscr{O}_{\mathrm{c}}: \mathrm{SLoc} \rightarrow \mathrm{SVec} \ldots \ldots \ldots \ldots \ldots \ldots \ldots$

5.2 Properties of the super-Green's operators . . . . . . . . . . . . . 20

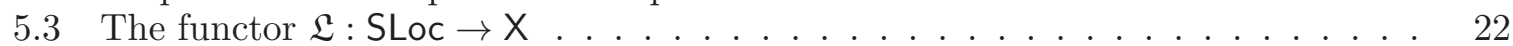

5.4 The quantization functor $\mathfrak{Q}: \mathrm{X} \rightarrow \mathrm{S}^{*} \mathrm{Alg} \ldots \ldots \ldots \ldots \ldots \ldots$

5.5 The locally covariant quantum field theory $\mathfrak{A}: \mathrm{SLoc} \rightarrow \mathrm{S}^{*} \mathrm{Alg} \ldots \ldots \ldots$

6 Axiomatic definition of enriched super-field theories 26

6.1 The monoidal category SSet of supersets . . . . . . . . . . . . . . 27

6.2 The SSet-category eSLoc . . . . . . . . . . . . . . . . . . . . 28

6.3 The SSet-functor $\mathfrak{e} \mathscr{O}: \mathrm{eSLoc}^{\mathrm{op}} \rightarrow \mathrm{eSVec} \ldots \ldots \ldots \ldots \ldots \ldots$

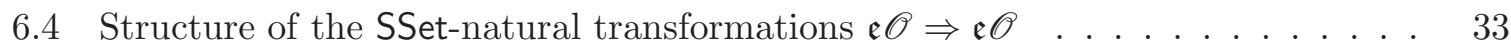

6.5 The definition . . . . . . . . . . . . . . . . . . . . 34

7 Construction of enriched super-quantum field theories 34

7.1 The SSet-functor $\mathfrak{e} \mathscr{O}_{\mathrm{c}}: \mathrm{eSLoc} \rightarrow$ eSVec $\ldots \ldots \ldots \ldots \ldots \ldots \ldots \ldots \ldots$

7.2 Enriched properties of the super-Green's operators . . . . . . . . . . . 35

7.3 The SSet-functor $\mathfrak{e} \mathfrak{L}: \mathrm{eSLoc} \rightarrow \mathrm{eX} \ldots \ldots \ldots \ldots \ldots \ldots \ldots$

7.4 The SSet-quantization functor $\mathfrak{e} \mathfrak{Q}: \mathrm{eX} \rightarrow \mathrm{eS}^{*} \mathrm{Alg} \ldots \ldots \ldots \ldots . \ldots \ldots$

7.5 The enriched locally covariant quantum field theory $\mathfrak{e A}: \mathrm{eSLoc} \rightarrow \mathrm{eS}{ }^{*} \mathrm{Alg}$. . . 39

8 Examples 41

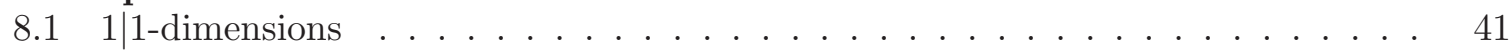

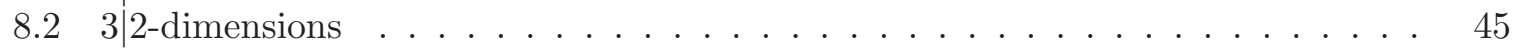

A Basics of enriched category theory 52

\section{Introduction and summary}

Over the past decades, supersymmetry and supergravity have been strongly vital research areas in theoretical and mathematical physics. On the one hand, supersymmetric extensions of the standard model provide interesting perspectives on particle physics and, on the other hand, supergravity arises as a low-energy limit of string theory and it might have potential applications to e.g. early universe cosmology. Regarded from the perspective of a quantum field theorist, interest in supersymmetry arises because of the well-known fact that certain supersymmetric quantum field theories enjoy unexpected renormalization properties, which collectively go under the name 'non-renormalization theorems', see e.g. GSR79, Sei93.

In contrast to the immense progress which theoretical physics has made during the past decades, mathematically rigorous developments of supersymmetric quantum field theories are quite rare. There are however some notable exceptions: In [BG06], Buchholz and Grundling 
address the nontrivial problems of implementing supersymmetry transformations into the $C^{*}$ algebraic framework of algebraic quantum field theory and constructing super-KMS states. The study of superconformal nets in two spacetime dimensions has been initiated by Capri, Kawahigashi and Longo in CKL08. Since then superconformal nets have been intensively developed, also with a focus on extended supersymmetry [CHKLX12]. Perturbative superconformal quantum field theories on a special class of (curved) spacetimes have been discussed quite recently by de Medeiros and Hollands dMH13, where also a perturbative non-renormalization theorem is rigorously proven. A formulation of (Euclidean) supersymmetric quantum field theories within the Atiyah-Segal approach and their connection to elliptic cohomology has been investigated by Stolz and Teichner, see e.g. the survey article [ST11].

In our work we shall study supersymmetric quantum field theories from the perspective of locally covariant quantum field theory [BFV03], which is a relatively modern extension of algebraic quantum field theory to curved spacetimes. In locally covariant quantum field theory, the focus is on the construction and analysis of functors from a category of spacetimes to a category of algebras, which are supposed to describe the assignment of observable algebras to spacetimes. Besides establishing a mathematical foundation for quantum field theory on curved spacetimes, locally covariant quantum field theory is essential for constructing perturbatively interacting models [HW01, HW02, BDF09]. Our aim is to extend carefully the formalism of locally covariant quantum field theory to the realm of supergeometry, focusing in the present work only on the case of non-interacting models. On the one hand, a solid understanding of non-interacting super-quantum field theories (super-QFTs) is a necessary prerequisite for constructing perturbative models and analyzing their renormalization behavior, especially concerning potential non-renormalization theorems. On the other hand, already simple examples of non-interacting super-QFTs indicate that the basic framework of locally covariant quantum field theory has to be generalized in order to be able to cope with the concept of supersymmetry transformations. In more detail, as we will show in this work, the framework of ordinary category theory, on which locally covariant quantum field theory is based, is insufficient to capture supersymmetry transformations on the level of the super-QFT functor. In particular, we observe that both the fermionic and the bosonic component fields are locally covariant quantum fields in the sense of [BFV03] (i.e. natural transformations to the super-QFT functor), which indicates that supersymmetry transformations are not appropriately described in this framework. Using techniques from enriched category theory, we shall propose a generalization of the framework in BFV03, which is general enough to capture supersymmetry transformations. Loosely speaking, we shall develop suitable categories of superspacetimes eSLoc and superalgebras eS*Alg that are enriched over the monoidal category of supersets and consider super-QFTs as enriched functors $\mathfrak{e} A$ : eSLoc $\rightarrow \mathrm{eS}^{*} A$ Ig between these enriched categories. Supersymmetry transformations are captured in terms of the higher superpoints of the morphism supersets in eSLoc and their action on the superalgebras of observables is dictated by the enriched functor $\mathfrak{e A}:$ eSLoc $\rightarrow \mathrm{eS}^{*} \mathrm{Alg}$. Our results will therefore clarify the structure of supersymmetry transformations in locally covariant quantum field theory, which will be essential for analyzing perturbative super-QFTs and their renormalization behavior in future works.

Let us outline the content of this paper: In Section 2 we shall give a self-contained and rather detailed introduction to those techniques of super-linear algebra and supergeometry that will be used in our work. This should allow readers who do not have a solid background in those fields to follow our constructions in the main part of this paper. In Section 3 we introduce super-Cartan structures on supermanifolds and study their properties. These structures have their origin in the superspace formulation of supergravity [WZ77] and they are used in our work in order to describe 'superspacetimes', which generalize Lorentz manifolds in ordinary quantum field theory. The dimension and the 'amount of supersymmetry' of a super-Cartan supermanifold is captured in its local model space, which we describe by using representation 
theoretic data corresponding to some spin group. We define a suitable category of super-Cartan supermanifolds and show that to any super-Cartan supermanifold there is a functorially associated oriented and time-oriented ordinary Lorentz manifold. This allows us to introduce a natural notion of the chronological and causal future/past in a super-Cartan supermanifold and therewith the concept of globally hyperbolic super-Cartan supermanifolds. In Section 4 we formulate a set of axioms to describe non-interacting super-field theories in an abstract way. According to our Definition 4.2, a super-field theory is specified by the following data: 1.) A choice of representation theoretic data that fixes the local model space of the super-Cartan supermanifolds. 2.) A full subcategory SLoc of the category of globally hyperbolic super-Cartan supermanifolds, which allows us later to implement constraints on the super-Cartan structures, e.g. the supergravity supertorsion constraints WZ77]. 3.) A suitable natural super-differential operator which encodes the dynamics of the super-field theory. We show in Section 5 that given any super-field theory as described above, one can construct a functor $\mathfrak{A}:$ SLoc $\rightarrow \mathrm{S}^{*} \mathrm{Alg}$ to the category of super-*-algebras which satisfies the axioms of locally covariant quantum field theory [BFV03] adapted to our supergeometric setting, cf. Theorem 5.11]. In other words, any super-field theory gives rise to a super-QFT. As in the case of ordinary quantum field theory, we first construct a functor $\mathfrak{L}: \mathrm{SLoc} \rightarrow \mathrm{X}$ to the category of super-symplectic spaces or the category of super-inner product spaces (depending on the representation theoretic data), which is then quantized by a quantization functor $\mathfrak{Q}: X \rightarrow S^{*} A l g$ that constructs super-canonical (anti)commutation relation algebras. We analyze the functor $\mathfrak{A}:$ SLoc $\rightarrow \mathrm{S}^{*}$ Alg and show that, in addition to the locally covariant quantum field which describes the linear superfield operators, the bosonic and fermionic component fields are also natural transformations in this framework. This is an undesirable feature which indicates that the framework developed in the Sections 4 and 5 does not capture supersymmetry transformations as those would mix the bosonic and fermionic components. We then solve this problem by making use of techniques from enriched category theory. In Section [6 we provide a stronger axiomatic framework for super-field theories by generalizing the category SLoc to a suitable category eSLoc which is enriched over the monoidal category of supersets SSet. The category SSet is defined as the functor category Fun(SPt ${ }^{\text {op }}$, Set), where SPt is the category of superpoints and op denotes the opposite category. Hence, a superset is a functor $\mathrm{SPt}^{\mathrm{op}} \rightarrow$ Set, which means that, in addition to its ordinary points, a superset has further content that is captured by its 'higher superpoints'. Loosely speaking, enriching the morphism sets in SLoc to the morphism supersets in eSLoc we obtain in addition to ordinary supermanifold morphisms $M \rightarrow M^{\prime}$ also supermanifold morphisms between the 'fattened' supermanifolds $\mathrm{pt}_{n} \times M \rightarrow \mathrm{pt}_{n} \times M^{\prime}$, where $\mathrm{pt}_{n}$ is any superpoint, that are able to capture supersymmetry transformations; indeed, the odd parameters which are used in the physics literature in order to parametrize supersymmetry transformations are elements in the structure sheaf $\Lambda_{n}=\bigwedge^{\bullet} \mathbb{R}^{n}$ (the Grassmann algebra) of $\mathrm{pt}_{n}$. It is important to notice that in this functorial approach we do not have to fix a superpoint (or equivalently a Grassmann algebra) from the outside, as it is typically done in the physics literature, but we are working functorially over the category of all superpoints. Similar techniques have been used before in order to describe super-mapping spaces between supermanifolds, see e.g. [Sac09, SW11, Han14]. In the enriched setting, the super-differential operators which govern the dynamics of the super-field theory should form an enriched natural transformation. We explicitly characterize these enriched natural transformations and show that they are in bijective correspondence to ordinary natural transformations (as used in Section (4) satisfying further conditions, which one may interpret as covariance conditions under supersymmetry transformations. This allows us to give a simple axiomatic characterization of enriched super-field theories in Definition 6.15. In Section 7 we show that any enriched super-field theory gives rise to an enriched super-QFT that we describe by an enriched functor $\mathfrak{e A}:$ eSLoc $\rightarrow \mathrm{eS}^{*}$ Alg to a suitable enriched category of super-*-algebras. We show that this enriched functor satisfies a generalization of the axioms of locally covariant quantum field the- 
ory, cf. Theorem 7.11. We further show that the enriched super-QFT has an enriched locally covariant quantum field (given by an enriched natural transformation) which describes the linear superfield operators. In contrast to the non-enriched theory studied in Section 5 , our enriched natural transformation does not decompose into the bosonic and fermionic component fields, which indicates that supersymmetry transformations are appropriately described within our enriched categorical framework. This is confirmed and illustrated in Section 8 by constructing and analyzing explicit examples of $1 \mid 1$ and $3 \mid 2$-dimensional enriched super-QFTs, together with the structure of supersymmetry transformations. Our 1|1-dimensional example is the usual superparticle and our 3|2-dimensional example is the free Wess-Zumino model on a class of curved super-Cartan supermanifolds. In Appendix A we collect some elementary definitions from enriched category theory which are needed in our work.

\section{Preliminaries on supergeometry}

We give a self-contained review of those aspects of super-linear algebra and supergeometry which we shall need for our work. For more details see e.g. CCF11 and DM99. In the following the ground field $\mathbb{K}$ will be either $\mathbb{R}$ or $\mathbb{C}$ and we set $\mathbb{Z}_{2}:=\{0,1\}$. Whenever there is no need to distinguish between the real and complex case, we shall drop the field $\mathbb{K}$ from our notations.

Super-vector spaces: A super-vector space is a $\mathbb{Z}_{2}$-graded vector space $V=V_{0} \oplus V_{1}$. We assign to the non-zero homogeneous elements $0 \neq v \in V_{i}$ the $\mathbb{Z}_{2}$-parity $|v|:=i \in \mathbb{Z}_{2}$, for $i=0,1$, and call elements in $V_{0}$ even and elements in $V_{1}$ odd. The superdimension (or simply dimension) of a super-vector space $V$ is denoted by $\operatorname{dim}(V):=\operatorname{dim}\left(V_{0}\right) \mid \operatorname{dim}\left(V_{1}\right)$. An example of an $n \mid m$-dimensional super-vector space is $\mathbb{K}^{n \mid m}:=\mathbb{K}^{n} \oplus \mathbb{K}^{m}$, with $n, m \in \mathbb{N}^{0}$. For simplicity, we shall denote $\mathbb{K}^{1 \mid 0}$ simply by $\mathbb{K}$. A super-vector space morphism $L: V \rightarrow V^{\prime}$ is a linear map which preserves the $\mathbb{Z}_{2}$-parity, i.e. $L\left(V_{i}\right) \subseteq V_{i}^{\prime}$ for $i=0,1$.

The category SVec of super-vector spaces has as objects all super-vector spaces and as morphisms all super-vector space morphisms. Recall that SVec is a monoidal category with tensor product functor $\otimes: \mathrm{SVec} \times \mathrm{SVec} \rightarrow \mathrm{SVec}$ and unit object $\mathbb{K}=\mathbb{K}^{1 \mid 0}$. Explicitly, the tensor product $V \otimes W$ of two super-vector spaces $V$ and $W$ is the ordinary tensor product $V \otimes W$ of vector spaces equipped with the $\mathbb{Z}_{2}$-grading

$$
\begin{aligned}
& (V \otimes W)_{0}:=\left(V_{0} \otimes W_{0}\right) \oplus\left(V_{1} \otimes W_{1}\right), \\
& (V \otimes W)_{1}:=\left(V_{0} \otimes W_{1}\right) \oplus\left(V_{1} \otimes W_{0}\right) .
\end{aligned}
$$

The tensor product of two SVec-morphisms is simply given by the tensor product of linear maps. The monoidal category SVec is symmetric with respect to the commutativity constraints

$$
\sigma_{V, W}: V \otimes W \longrightarrow W \otimes V, \quad v \otimes w \longmapsto(-1)^{|v||w|} w \otimes v .
$$

Moreover, it is closed with internal hom-objects given by the vector space $\underline{\operatorname{Hom}}(V, W)$ of all linear maps $L: V \rightarrow W$ equipped with the obvious $\mathbb{Z}_{2}$-grading; $L \in \underline{\operatorname{Hom}}(V, W)$ is even/odd if it preserves/reverses the $\mathbb{Z}_{2}$-parity.

Given an object $V=V_{0} \oplus V_{1}$ in SVec, a super-vector subspace is a vector subspace $W \subseteq V$ together with a $\mathbb{Z}_{2}$-grading $W=W_{0} \oplus W_{1}$ such that $W_{i}$ is a vector subspace of $V_{i}$, for $i=0,1$. We then may form the quotient super-vector space $V / W:=V_{0} / W_{0} \oplus V_{1} / W_{1}$, which comes together with a canonical SVec-morphism $V \rightarrow V / W$ assigning equivalence classes. 
Superalgebras: A (unital and associative) superalgebra is an algebra object in SVec. Explicitly, this means that a superalgebra is an object $A$ in SVec together with two SVec-morphisms $\mu_{A}: A \otimes A \rightarrow A$ (called product) and $\eta_{A}: \mathbb{K} \rightarrow A$ (called unit), such that the diagrams
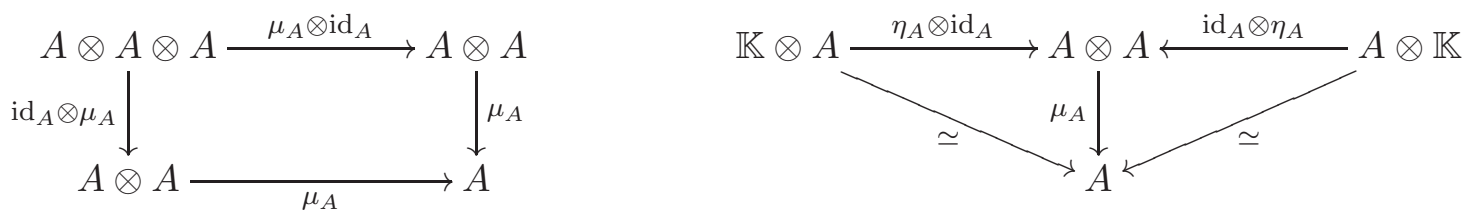

in SVec commute. We shall often denote the products by juxtaposition, i.e. $\mu_{A}\left(a_{1} \otimes a_{2}\right)=a_{1} a_{2}$, and the unit element by $\eta_{A}(1)=\mathbb{1}$. An example of a (real) superalgebra is the Grassmann algebra $\Lambda_{n}:=\bigwedge^{\bullet} \mathbb{R}^{n}$, for $n \in \mathbb{N}^{0}$, with product given by the wedge product and unit element by $\mathbb{1}=1 \in \mathbb{R}=\bigwedge^{0} \mathbb{R}^{n} \subseteq \bigwedge^{\bullet} \mathbb{R}^{n}$. A superalgebra morphism $\kappa: A \rightarrow A^{\prime}$ is a SVec-morphism which preserves products and units, i.e. $\mu_{A^{\prime}} \circ(\kappa \otimes \kappa)=\kappa \circ \mu_{A}$ and $\eta_{A^{\prime}}=\kappa \circ \eta_{A}$.

We denote the category of superalgebras by SAlg and notice that it is a monoidal category: The tensor product $A \otimes B$ of two superalgebras is the super-vector space $A \otimes B$ equipped with the following product and unit

$$
\begin{gathered}
\mu_{A \otimes B}:=\left(\mu_{A} \otimes \mu_{B}\right) \circ\left(\operatorname{id}_{A} \otimes \sigma_{B, A} \otimes \operatorname{id}_{B}\right): A \otimes B \otimes A \otimes B \longrightarrow A \otimes B \\
\eta_{A \otimes B}:=\eta_{A} \otimes \eta_{B}: \mathbb{K} \otimes \mathbb{K} \simeq \mathbb{K} \longrightarrow A \otimes B .
\end{gathered}
$$

Explicitly, we have for the product $\left(a_{1} \otimes b_{1}\right)\left(a_{2} \otimes b_{2}\right)=(-1)^{\left|a_{2}\right|\left|b_{1}\right|}\left(a_{1} a_{2}\right) \otimes\left(b_{1} b_{2}\right)$, for all homogeneous $a_{1}, a_{2} \in A$ and $b_{1}, b_{2} \in B$, and for the unit $\mathbb{1}_{A \otimes B}=\mathbb{1}_{A} \otimes \mathbb{1}_{B}$. The tensor product of two SAlg-morphisms is simply given by the tensor product of linear maps.

We shall require some special classes of superalgebras. A superalgebra $A$ is called supercommutative if the product is compatible with the commutativity constraint, i.e. $\mu_{A} \circ \sigma_{A, A}=\mu_{A}$. Notice that supercommutative superalgebras form a monoidal subcategory of SAlg, which is symmetric with respect to the commutativity constraints induced by SVec. Moreover, for a supercommutative superalgebra $A$ the product $\mu_{A}: A \otimes A \rightarrow A$ is a SAlg-morphism with respect to the tensor product superalgebra structure on $A \otimes A$.

Let us now consider superalgebras over $\mathbb{C}$. A super-*-algebra is a superalgebra $A$ over $\mathbb{C}$ together with an even $\mathbb{C}$-antilinear map $*_{A}: A \rightarrow A$ (called superinvolution) which satisfies $*_{A} \circ \eta_{A}=\eta_{A}$ and $*_{A} \circ \mu_{A}=\mu_{A} \circ \sigma_{A, A} \circ\left(*_{A} \otimes_{\mathbb{C}} *_{A}\right)$. Explicitly, these conditions read as $\mathbb{1}^{*}=\mathbb{1}$ and $\left(a_{1} a_{2}\right)^{*}=(-1)^{\left|a_{1}\right|\left|a_{2}\right|} a_{2}^{*} a_{1}^{*}$, for homogeneous elements $a_{1}, a_{2} \in A$. A super-*-algebra morphism $\kappa: A \rightarrow A^{\prime}$ is a SAlg-morphism satisfying $\kappa \circ *_{A}=*_{A^{\prime}} \circ \kappa$. We denote the category of super- $*-$ algebras by $\mathrm{S}^{*} \mathrm{Alg}$ and notice that it is a monoidal category; the superinvolution on the tensor product $A \otimes_{\mathbb{C}} B$ of two super-*-algebras is defined component-wise, i.e. $\left(a \otimes_{\mathbb{C}} b\right)^{*}:=a^{*} \otimes_{\mathbb{C}} b^{*}$.

Super-Lie algebras: A super-Lie algebra is a Lie algebra object in SVec. Explicitly, a superLie algebra is an object $\mathfrak{g}$ in SVec together with a SVec-morphism $[\cdot, \cdot]_{\mathfrak{g}}: \mathfrak{g} \otimes \mathfrak{g} \rightarrow \mathfrak{g}$ (called super-Lie bracket) which satisfies the super-skew symmetry condition

$$
[\cdot, \cdot]_{\mathfrak{g}} \circ\left(\operatorname{id}_{\mathfrak{g} \otimes \mathfrak{g}}+\sigma_{\mathfrak{g}, \mathfrak{g}}\right)=0
$$

and the super-Jacobi identity

$$
\left[\cdot,[\cdot, \cdot]_{\mathfrak{g}}\right]_{\mathfrak{g}} \circ\left(\mathrm{id}_{\mathfrak{g} \otimes \mathfrak{g} \otimes \mathfrak{g}}+\sigma_{\mathfrak{g}, \mathfrak{g} \otimes \mathfrak{g}}+\sigma_{\mathfrak{g} \otimes \mathfrak{g}, \mathfrak{g}}\right)=0 .
$$

A super-Lie algebra morphism $L: \mathfrak{g} \rightarrow \mathfrak{g}^{\prime}$ is a SVec-morphism which preserves the super-Lie brackets, i.e. $[\cdot, \cdot]_{\mathfrak{g}^{\prime}} \circ(L \otimes L)=L \circ[\cdot, \cdot]_{\mathfrak{g}}$. 
Supermodules and the Berezinian: Let $A$ be a superalgebra. A left $A$-supermodule is a left module object in SVec. Explicitly, a left $A$-supermodule is an object $V$ in SVec together with a SVec-morphism $l_{V}: A \otimes V \rightarrow V$ (called left $A$-action), such that the diagrams
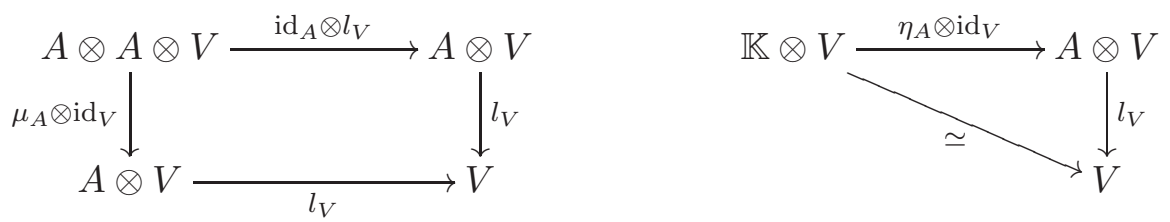

in SVec commute. A right A-supermodule is defined similarly and an A-bisupermodule is a left and right $A$-supermodule with commuting left and right $A$-actions. If $A$ is a supercommutative superalgebra, then any left $A$-supermodule $V$ is also a right $A$-supermodule with right $A$ action $r_{V}:=l_{V} \circ \sigma_{V, A}: V \otimes A \rightarrow V$ and, vice versa, any right $A$-supermodule is also a left $A$-supermodule with left $A$-action $l_{V}:=r_{V} \circ \sigma_{A, V}: A \otimes V \rightarrow V$. Notice that these left and right $A$-actions are compatible, hence $V$ is an $A$-bisupermodule. We shall often denote the left and right $A$-actions simply by juxtaposition, i.e. $l_{V}(a \otimes v)=a v$ and $r_{V}(v \otimes a)=v a$. A left A-supermodule morphism $L: V \rightarrow V^{\prime}$ is a SVec-morphism which preserves the left $A$-actions, i.e. $l_{V^{\prime}} \circ\left(\operatorname{id}_{A} \otimes L\right)=L \circ l_{V}$. We denote the category of left $A$-supermodules by $A$-SMod. In the case of $A$ being supercommutative, $A$-SMod is a monoidal category with tensor product functor $\otimes_{A}: A$-SMod $\times A$-SMod $\rightarrow A$-SMod (taking tensor products over $A$ ) and unit object $A$ (regarded as a left $A$-supermodule with left $A$-action given by the product $\mu_{A}$ ). Again for $A$ being supercommutative, the monoidal category $A$-SMod is also symmetric with commutativity constraints induced by those in SVec and closed with internal hom-objects given by the left $A$-supermodules $\underline{\operatorname{Hom}}_{A}(V, W)$ of all right $A$-linear maps $L: V \rightarrow W$ equipped with the obvious $\mathbb{Z}_{2}$-grading; $L \in \underline{\operatorname{Hom}}_{A}(V, W)$ is even/odd if it preserves/reverses the $\mathbb{Z}_{2}$-parity.

A free left $A$-supermodule of dimension $n \mid m$ is a left $A$-supermodule $V$ for which there exists a basis of $n \in \mathbb{N}^{0}$ even elements $\left\{e_{1}, \ldots, e_{n}\right\}$ and $m \in \mathbb{N}^{0}$ odd elements $\left\{\epsilon_{1}, \ldots, \epsilon_{m}\right\}$, such that

$$
\begin{aligned}
& V_{0}=\operatorname{span}_{A_{0}}\left\{e_{1}, \ldots, e_{n}\right\} \oplus \operatorname{span}_{A_{1}}\left\{\epsilon_{1}, \ldots, \epsilon_{m}\right\}, \\
& V_{1}=\operatorname{span}_{A_{1}}\left\{e_{1}, \ldots, e_{n}\right\} \oplus \operatorname{span}_{A_{0}}\left\{\epsilon_{1}, \ldots, \epsilon_{m}\right\} .
\end{aligned}
$$

The collection $\left\{e_{1}, \ldots, e_{n+m}\right\}:=\left\{e_{1}, \ldots, e_{n}, \epsilon_{1}, \ldots, \epsilon_{m}\right\}$ of elements in $V$ is called an adapted basis for $V$. Notice that any free left $A$-supermodule of dimension $n \mid m$ is isomorphic (in the category $A$-SMod) to the standard free left $A$-supermodule $A^{n \mid m}:=A \otimes \mathbb{K}^{n \mid m}$ with the obvious left $A$-action. The $A$-SMod-morphisms between two free left $A$-supermodules can be represented in terms of matrices with entries in $A$. Explicitly, let $L: V \rightarrow V^{\prime}$ be any $A$-SModmorphism between an $n \mid m$-dimensional free left $A$-supermodule $V$ and an $n^{\prime} \mid m^{\prime}$-dimensional free left $A$-supermodule $V^{\prime}$. Making use of any adapted bases for $V$ and $V^{\prime}$ we define the elements $\left\{L_{i}^{j} \in A: i=1, \ldots, n+m, j=1, \ldots, n^{\prime}+m^{\prime}\right\}$ via $L\left(e_{i}\right)=\sum_{j=1}^{n^{\prime}+m^{\prime}} L_{i}^{j} e_{j}^{\prime}$, which can be arranged in an $(n+m) \times\left(n^{\prime}+m^{\prime}\right)$-matrix of the form

$$
\underline{\underline{L}}=\left(\begin{array}{ll}
L_{1} & L_{2} \\
L_{3} & L_{4}
\end{array}\right)
$$

where $L_{1}$ is an $n \times n^{\prime}$-matrix with entries in $A_{0}, L_{2}$ is an $n \times m^{\prime}$-matrix with entries in $A_{1}, L_{3}$ is an $m \times n^{\prime}$-matrix with entries in $A_{1}$ and $L_{4}$ is an $m \times m^{\prime}$-matrix with entries in $A_{0}$.

Let now $A$ be a supercommutative superalgebra and $V$ any free left $A$-supermodule. Denoting the group of $A$-SMod-automorphisms of $V$ by GL $(V)$, there exists a group homomorphism (called the Berezinian) to the group of invertible elements in $A$

$$
\text { Ber : } \operatorname{GL}(V) \longrightarrow A_{0}^{\times}, L \longmapsto \operatorname{Ber}(L)=\operatorname{det}\left(L_{1}-L_{2} L_{4}^{-1} L_{3}\right) \operatorname{det}\left(L_{4}\right)^{-1},
$$


where we have made use of an arbitrary adapted basis for $V$ (the Berezinian does not depend on the choice of adapted basis). One can easily check that the Berezinian is multiplicative, i.e. $\operatorname{Ber}\left(L^{\prime} L\right)=\operatorname{Ber}\left(L^{\prime}\right) \operatorname{Ber}(L)$, for all $L, L^{\prime} \in \mathrm{GL}(V)$. Moreover, we may assign to any free left $A$-supermodule $V$ of dimension $n \mid m$ its Berezinian left $A$-supermodule $\operatorname{Ber}(V)$ that is defined as follows: $\operatorname{Ber}(V)$ is the free left $A$-supermodule that is generated by the elements $\left[e_{1}, \ldots, e_{n+m}\right]$, for all adapted bases $\left\{e_{1}, \ldots, e_{n+m}\right\}$ for $V$, subject to the relations

$$
\left[L\left(e_{1}\right), \ldots, L\left(e_{n+m}\right)\right]=\operatorname{Ber}(L)\left[e_{1}, \ldots, e_{n+m}\right],
$$

for all $L \in \mathrm{GL}(V)$. We declare the elements $\left[e_{1}, \ldots, e_{n+m}\right]$ to be even if $m \in 2 \mathbb{N}^{0}$ or to be odd if $m \in 2 \mathbb{N}^{0}+1$. Since any two adapted bases for $V$ can be related by a GL(V)transformation, it is clear that $\operatorname{Ber}(V)$ is a free left $A$-supermodule of dimension $1 \mid 0$ if $m \in 2 \mathbb{N}^{0}$ or dimension $0 \mid 1$ if $m \in 2 \mathbb{N}^{0}+1$. Notice that any choice of adapted basis $\left\{e_{1}, \ldots, e_{n+m}\right\}$ for $V$ defines an adapted basis $\left[e_{1}, \ldots, e_{n+m}\right]$ for $\operatorname{Ber}(V)$. The assignment of the Berezinian left $A$-supermodules is functorial: Given any $A$-SMod-isomorphism $L: V \rightarrow V^{\prime}$ between two free left $A$-supermodules $V$ and $V^{\prime}$ we define an $A$-SMod-isomorphism $\operatorname{Ber}(L): \operatorname{Ber}(V) \rightarrow \operatorname{Ber}\left(V^{\prime}\right)$ by setting for any adapted basis $\left[e_{1}, \ldots, e_{n+m}\right]$ for $\operatorname{Ber}(V)$

$$
\operatorname{Ber}(L)\left(\left[e_{1}, \ldots, e_{n+m}\right]\right):=\left[L\left(e_{1}\right), \ldots, L\left(e_{n+m}\right)\right],
$$

and extending $\operatorname{Ber}(L)$ as an $A$-SMod-morphism.

Supermanifolds: In the following let $\mathbb{K}=\mathbb{R}$. A superspace is a pair $M:=\left(\widetilde{M}, \mathscr{O}_{M}\right)$ consisting of a topological space $\widetilde{M}$ (which we always assume to be second-countable and Hausdorff) and a sheaf of supercommutative superalgebras $\mathscr{O}_{M}$ on $\widetilde{M}$ (called the structure sheaf). We denote the restriction SAlg-morphisms in the structure sheaf $\mathscr{O}_{M}$ by $\operatorname{res}_{U, V}: \mathscr{O}_{M}(U) \rightarrow \mathscr{O}_{M}(V)$, for all open $V \subseteq U \subseteq \widetilde{M}$, and the global sections of the structure sheaf simply by $\mathscr{O}(M):=$ $\mathscr{O}_{M}(\widetilde{M})$. A superspace morphism $\chi: M \rightarrow M^{\prime}$ is a pair $\chi:=\left(\widetilde{\chi}, \chi^{*}\right)$ consisting of a continuous map $\tilde{\chi}: \widetilde{M} \rightarrow \widetilde{M^{\prime}}$ and a morphism of sheaves of superalgebras $\chi^{*}: \mathscr{O}_{M^{\prime}} \rightarrow \widetilde{\chi}_{*}\left(\mathscr{O}_{M}\right)$, where $\tilde{\chi}_{*}\left(\mathscr{O}_{M}\right)$ denotes the direct image sheaf. Explicitly, $\chi^{*}: \mathscr{O}_{M^{\prime}} \rightarrow \widetilde{\chi}_{*}\left(\mathscr{O}_{M}\right)$ consists of assigning to any open $U \subseteq \widetilde{M^{\prime}}$ a SAlg-morphism $\chi_{U}^{*}: \mathscr{O}_{M^{\prime}}(U) \rightarrow \mathscr{O}_{M}\left(\widetilde{\chi}^{-1}(U)\right)$, such that the diagram

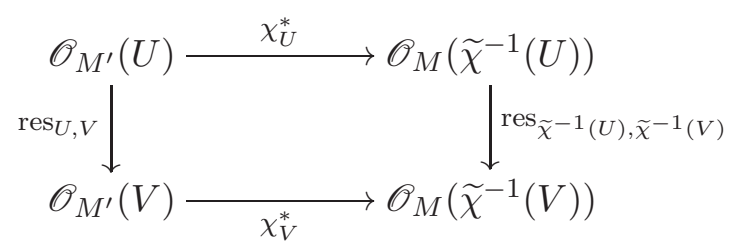

in SAlg commutes, for all open $V \subseteq U \subseteq \widetilde{M^{\prime}}$. For notational simplicity we shall denote the SAlg-morphism of global sections by $\chi^{*}:=\chi_{\widetilde{M}^{\prime}}^{*}: \mathscr{O}\left(M^{\prime}\right) \rightarrow \mathscr{O}(M)$. An example of a superspace is $\mathbb{R}^{n \mid m}:=\left(\mathbb{R}^{n}, C_{\mathbb{R}^{n}}^{\infty} \otimes \bigwedge^{\bullet} \mathbb{R}^{m}\right)$, for $n, m \in \mathbb{N}^{0}$, which we denote with the usual abuse of notation by the same symbol as the standard super-vector space above.

A supermanifold of dimension $n \mid m$ is a superspace $M=\left(\widetilde{M}, \mathscr{O}_{M}\right)$ that is locally isomorphic to $\mathbb{R}^{n \mid m}$, with $n, m \in \mathbb{N}^{0}$ fixed. In more detail, given any $p \in \widetilde{M}$, there exists an open neighborhood $V \subseteq \widetilde{M}$ of $p$, such that $V$ is homeomorphic to an open subset $U \subseteq \mathbb{R}^{n}$ and such that the restricted sheaves of superalgebras $\left.\mathscr{O}_{M}\right|_{V}$ and $\left.C_{\mathbb{R}^{n}}^{\infty}\right|_{U} \otimes \bigwedge^{\bullet} \mathbb{R}^{m}$ are isomorphic. Taking the standard coordinates $\left(x^{1}, \ldots, x^{n}\right)$ of $\mathbb{R}^{n}$ and the standard coordinates $\left(\theta^{1}, \ldots, \theta^{m}\right)$ of $\mathbb{R}^{m}$, the sheaf isomorphism $\left.\left.\mathscr{O}_{M}\right|_{V} \simeq C_{\mathbb{R}^{n}}^{\infty}\right|_{U} \otimes \bigwedge^{\bullet} \mathbb{R}^{m}$ induces local coordinate functions of $M$ in $V$, which we denote by the same symbols $\left(x^{1}, \ldots, x^{n+m}\right):=\left(x^{1}, \ldots, x^{n}, \theta^{1}, \ldots, \theta^{m}\right)$, hence suppressing the isomorphism. Notice that the first $n$ coordinate functions $x^{i} \in \mathscr{O}_{M}(V)$, for $i=1, \ldots, n$, are even and that the last $m$ coordinate functions $x^{n+i} \in \mathscr{O}_{M}(V)$, for $i=1, \ldots, m$, 
are odd. It is clear that the superspaces $\mathbb{R}^{n \mid m}=\left(\mathbb{R}^{n}, C_{\mathbb{R}^{n}}^{\infty} \otimes \bigwedge^{\bullet} \mathbb{R}^{m}\right)$ are $n \mid m$-dimensional supermanifolds, for all $n, m \in \mathbb{N}^{0}$. Moreover, given any $n \mid m$-dimensional supermanifold $M=$ $\left(\widetilde{M}, \mathscr{O}_{M}\right)$ and any open $U \subseteq \widetilde{M}$, then $\left.M\right|_{U}:=\left(U,\left.\mathscr{O}_{M}\right|_{U}\right)$ is an $n \mid m$-dimensional supermanifold, which we call the open subsupermanifold determined by $U$. A supermanifold morphism is a superspace morphism between supermanifolds. It is well known that supermanifold morphisms $\chi: M \rightarrow M^{\prime}$ are already uniquely specified by their associated SAlg-morphisms $\chi^{*}: \mathscr{O}\left(M^{\prime}\right) \rightarrow$ $\mathscr{O}(M)$ on global sections of the structure sheaf, see e.g. [CCF11, Proposition 4.6.1].

We denote the category of supermanifolds by SMan and notice that it is a monoidal category with monoidal bifunctor $\times:$ SMan $\times$ SMan $\rightarrow$ SMan and unit object pt $:=(\{\star\}, \mathbb{R})$. The monoidal bifunctor is defined as follows, cf. [CCF11, Chapter 4.5]: To any pair $\left(M, M^{\prime}\right)$ of objects in SMan it assigns the supermanifold $M \times M^{\prime}:=\left(\widetilde{M} \times \widetilde{M}^{\prime}, \mathscr{O}_{M \times M^{\prime}}\right)$, where the sheaf of superalgebras $\mathscr{O}_{M \times M^{\prime}}$ is defined on the rectangular open subsets $U \times V \subseteq \widetilde{M} \times \widetilde{M}^{\prime}$ by $\mathscr{O}_{M \times M^{\prime}}(U \times V):=\mathscr{O}_{M}(U) \widehat{\otimes} \mathscr{O}_{M^{\prime}}(V)$ and extended to all open subsets of $\widetilde{M} \times \widetilde{M^{\prime}}$ by a standard construction. Here $\widehat{\otimes}$ denotes the completed tensor product in the projective tensor topology corresponding to the standard Fréchet topologies on $\mathscr{O}_{M}(U)$ and $\mathscr{O}_{M^{\prime}}(V)$. To any pair $(\chi: M \rightarrow$ $N, \chi^{\prime}: M^{\prime} \rightarrow N^{\prime}$ ) of SMan-morphisms it assigns the SMan-morphism $\chi \times \chi^{\prime}: M \times M^{\prime} \rightarrow N \times N^{\prime}$ given by $\tilde{\chi} \times \widetilde{\chi^{\prime}}: \widetilde{M} \times \widetilde{M^{\prime}} \rightarrow \widetilde{N} \times \widetilde{N^{\prime}}$ and $\chi^{*} \widehat{\otimes} \chi^{\prime *}: \mathscr{O}_{N \times N^{\prime}} \rightarrow\left(\widetilde{\chi} \times \widetilde{\chi}^{\prime}\right)_{*}\left(\mathscr{O}_{M \times M^{\prime}}\right)$, which is uniquely determined by continuous extension of the usual tensor product morphism $\chi^{*} \otimes \chi^{\prime *}$.

Let us recall that any $n \mid m$-dimensional supermanifold $M$ has an associated reduced $n$ dimensional ordinary manifold. Let us denote by $J_{M}$ the super-ideal sheaf of nilpotents in $\mathscr{O}_{M}$, i.e. $J_{M}(U):=\left\{f \in \mathscr{O}_{M}(U): f\right.$ is nilpotent $\}$ for all open $U \subseteq \widetilde{M}$. The reduced manifold is defined to be the $n \mid 0$-dimensional supermanifold $\left(\widetilde{M}, \mathscr{O}_{M} / J_{M}\right)$, which for notational convenience we shall simply denote by $\widetilde{M}$. For any SMan-morphism $\chi: M \rightarrow M^{\prime}$ the underlying continuous map $\widetilde{\chi}: \widetilde{M} \rightarrow \widetilde{M^{\prime}}$ is smooth with respect to the reduced manifold structures on $\widetilde{M}$ and $\widetilde{M^{\prime}}$. Hence, the assignment $M \mapsto \widetilde{M}$ and $\left(\chi: M \rightarrow M^{\prime}\right) \mapsto\left(\widetilde{\chi}: \widetilde{M} \rightarrow \widetilde{M^{\prime}}\right)$ is a functor from SMan to the category of manifolds. Finally, recall that for any supermanifold $M$ there is a closed embedding $\iota \widetilde{M, M}: \widetilde{M} \rightarrow M$ of the reduced manifold (regarded as an $n \mid 0$-dimensional supermanifold) into the supermanifold $M$. Explicitly, we have that $\widetilde{\iota}_{\widetilde{M}, M}=\mathrm{id}_{\widetilde{M}}$ is the identity map and that $\iota_{\widetilde{M}, M}^{*}: \mathscr{O}_{M} \rightarrow \mathscr{O}_{M} / J_{M}$ is the projection taking equivalence classes. Given any SMan-morphism $\chi: M \rightarrow M^{\prime}$ the diagram

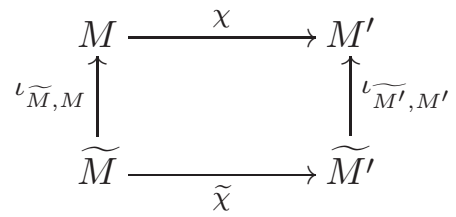

in SMan commutes.

Superderivations and super-differential operators: Let $M=\left(\widetilde{M}, \mathscr{O}_{M}\right)$ be any object in SMan. For any open $U \subseteq \widetilde{M}$, a homogeneous superderivation (or super-vector field) on $\mathscr{O}_{M}(U)$ is a homogeneous $X \in \underline{\operatorname{Hom}}\left(\mathscr{O}_{M}(U), \mathscr{O}_{M}(U)\right)$, such that

$$
X(f g)=X(f) g+(-1)^{|X||f|} f X(g),
$$

for all homogeneous $f, g \in \mathscr{O}_{M}(U)$. The collection of all superderivations on $\mathscr{O}_{M}(U)$ forms a super-vector subspace $\operatorname{Der}_{M}(U)$ of $\underline{\operatorname{Hom}}\left(\mathscr{O}_{M}(U), \mathscr{O}_{M}(U)\right)$, which is further a left $\mathscr{O}_{M}(U)$ supermodule via $(f X)(g):=f X(g)$, for all $f, g \in \mathscr{O}_{M}(U)$ and $X \in \operatorname{Der}_{M}(U)$. As in the case of ordinary manifolds, the superderivations $\operatorname{Der}_{M}(U)$ also form a super-Lie algebra with super-Lie bracket given by the supercommutator $[X, Y]:=X \circ Y-(-1)^{|X|}|Y| Y \circ X$, for all 
homogeneous $X, Y \in \operatorname{Der}_{M}(U)$. The assignment $U \mapsto \operatorname{Der}_{M}(U)$ (together with suitable restriction morphisms) is a sheaf of left $\mathscr{O}_{M}$-supermodules on $\widetilde{M}$, which is called the superderivation sheaf $\operatorname{Der}_{M}$.

Note that superderivations are local in the following sense: Let $U \subseteq \widetilde{M}$ be any open subset. The support of $f \in \mathscr{O}_{M}(U)$ is the closed subset of $\widetilde{M}$ that is defined by

$$
\operatorname{supp}(f):=U \backslash \bigcup\left\{V: V \subseteq U \text { is open and } \operatorname{res}_{U, V}(f)=0\right\} .
$$

It then holds true that $\operatorname{supp}(X(f)) \subseteq \operatorname{supp}(f)$, for all $X \in \operatorname{Der}_{M}(U)$ and $f \in \mathscr{O}_{M}(U)$.

The superderivation sheaf $\operatorname{Der}_{M}$ of any object $M$ in SMan is a sheaf of locally free left $\mathscr{O}_{M}$-supermodules of the same dimension $n \mid m$ as the supermanifold $M$. In local coordinates $\left(x^{1}, \ldots, x^{n+m}\right)$ of $M$ in $V$ an adapted basis for $\operatorname{Der}_{M}(V)$ is given by the partial derivatives $\left\{\partial_{1}, \ldots, \partial_{n+m}\right\}$, which are defined by $\partial_{i} x^{j}=\delta_{i}^{j}$, for all $i, j=1, \ldots, n+m$. Notice that the first $n$ partial derivatives are even and the last $m$ partial derivatives are odd.

We introduce super-differential operators via the usual recursive procedure: For any open $U \subseteq \widetilde{M}$, we have a SVec-morphism $\mathscr{O}_{M}(U) \rightarrow \underline{\operatorname{Hom}}\left(\mathscr{O}_{M}(U), \mathscr{O}_{M}(U)\right)$ by assigning to any $g \in \mathscr{O}_{M}(U)$ the linear map (denoted by the same symbol) $g: \mathscr{O}_{M}(U) \rightarrow \mathscr{O}_{M}(U), f \mapsto g f$. The image of this SVec-morphism is denoted by $\operatorname{DiffOp}_{M}^{0}(U)$ and it is called the super-differential operators of order zero. The super-differential operators of order $k \geq 1$ are then defined recursively via

$$
\operatorname{DiffOp}_{M}^{k}(U):=\left\{L \in \underline{\operatorname{Hom}}\left(\mathscr{O}_{M}(U), \mathscr{O}_{M}(U)\right):[L, g] \in \operatorname{DiffOp}_{M}^{k-1}(U), \quad \forall g \in \mathscr{O}_{M}(U)\right\},
$$

where the bracket denotes the supercommutator. The super-vector space of super-differential operators on $\mathscr{O}_{M}(U)$ is defined by $\operatorname{DiffOp}_{M}(U):=\bigcup_{k \geq 0} \operatorname{DiffOp}_{M}^{k}(U)$. Notice that $\operatorname{DiffOp}_{M}(U)$ carries the structure of a (not necessarily supercommutative) superalgebra with product given by the composition o of linear maps $\underline{\operatorname{Hom}}\left(\mathscr{O}_{M}(U), \mathscr{O}_{M}(U)\right)$. The assignment $U \mapsto \operatorname{DiffOp}_{M}(U)$ (together with suitable restriction morphisms) is a sheaf of superalgebras on $\widetilde{M}$, which is called the sheaf of super-differential operators DiffOp ${ }_{M}$. Super-differential operators are local, i.e. given any open $U \subseteq \widetilde{M}$ we have that $\operatorname{supp}(D(f)) \subseteq \operatorname{supp}(f)$, for any $D \in \operatorname{DiffOp}_{M}(U)$ and $f \in \mathscr{O}_{M}(U)$. Any superderivation is a super-differential operator of order 1 .

Super-differential forms: Let $M=\left(\widetilde{M}, \mathscr{O}_{M}\right)$ be any object in SMan. For any open $U \subseteq \widetilde{M}$ we define the left $\mathscr{O}_{M}(U)$-supermodule of super-one-forms on $\mathscr{O}_{M}(U)$ as the dual of the left $\mathscr{O}_{M}(U)$-supermodule $\operatorname{Der}_{M}(U)$, i.e. $\Omega_{M}^{1}(U):=\underline{\operatorname{Hom}}_{\mathscr{O}_{M}(U)}\left(\operatorname{Der}_{M}(U), \mathscr{O}_{M}(U)\right)$. The assignment $U \mapsto \Omega_{M}^{1}(U)$ (together with suitable restriction morphisms) is a sheaf of left $\mathscr{O}_{M}$-supermodules on $\widetilde{M}$ that we shall denote by $\Omega_{M}^{1}=\underline{\operatorname{Hom}}_{\mathscr{O}_{M}}\left(\operatorname{Der}_{M}, \mathscr{O}_{M}\right)$. For simplifying the notation we denote the left $\mathscr{O}(M)$-supermodule of global sections of $\Omega_{M}^{1}$ by $\Omega^{1}(M)$. The canonical evaluation of elements in $\underline{\operatorname{Hom}}_{\mathscr{O}_{M}(U)}\left(\operatorname{Der}_{M}(U), \mathscr{O}_{M}(U)\right)$ on superderivations $\operatorname{Der}_{M}(U)$ defines a sheaf morphism ev $: \Omega_{M}^{1} \otimes_{\mathscr{O}_{M}} \operatorname{Der}_{M} \rightarrow \mathscr{O}_{M}$, which due to the commutativity constraint $\sigma$ in $\mathscr{O}_{M}$-SMod can also be understood as a pairing

$$
\langle\cdot, \cdot\rangle:=\operatorname{ev} \circ \sigma_{\operatorname{Der}_{M}, \Omega_{M}^{1}}: \operatorname{Der}_{M} \otimes_{\mathscr{O}_{M}} \Omega_{M}^{1} \longrightarrow \mathscr{O}_{M}
$$

where the superderivations are on the left. (Moving $\operatorname{Der}_{M}$ to the left will lead to more convenient sign conventions for the differential below.) Explicitly, given any homogeneous $\omega \in \Omega_{M}^{1}(U)$ and $X \in \operatorname{Der}_{M}(U)$, the pairing reads as $\langle X, \omega\rangle=(-1)^{|\omega||X|} \omega(X)$. The differential d $: \mathscr{O}_{M} \rightarrow \Omega_{M}^{1}$ is the sheaf morphism defined by the condition $\langle X, \mathrm{~d} f\rangle:=X(f)$, for all $X \in \operatorname{Der}_{M}(U)$ and $f \in \mathscr{O}_{M}(U)$, where $U \subseteq \widetilde{M}$ is any open subset. We notice that $\Omega_{M}^{1}$ is a sheaf of locally free left $\mathscr{O}_{M}$-supermodules of the same dimension $n \mid m$ as the supermanifold $M$. 
Explicitly, in local coordinates $\left(x^{1}, \ldots, x^{n+m}\right)$ of $M$ in $V$ an adapted basis for $\Omega_{M}^{1}(V)$ is given by the differentials $\left\{\mathrm{d} x^{1}, \ldots, \mathrm{d} x^{n+m}\right\}$, which can also be characterized by the duality relations $\left\langle\partial_{i}, \mathrm{~d} x^{j}\right\rangle=\delta_{i}^{j}$, for all $i, j=1, \ldots, n+m$. Notice that the first $n$ differentials are even and that the last $m$ differentials are odd.

Super-one-forms can be pulled back along SMan-morphisms $\chi: M \rightarrow M^{\prime}$. In our work we shall only need the pull-back for the special case where $\widetilde{\chi}(\widetilde{M}) \subseteq \widetilde{M}^{\prime}$ is open and $\chi: M \rightarrow$ $\left.M^{\prime}\right|_{\widetilde{\chi}(\widetilde{M})}$ is a SMan-isomorphism. Let us first assume that $\chi: M \rightarrow M^{\prime}$ is a SMan-isomorphism. Then $\chi_{U}^{*}: \mathscr{O}_{M^{\prime}}(U) \rightarrow \mathscr{O}_{M}\left(\tilde{\chi}^{-1}(U)\right)$ is a SAlg-isomorphism and we can define a push-forward of superderivations by

$$
\chi_{* U}: \operatorname{Der}_{M}\left(\tilde{\chi}^{-1}(U)\right) \longrightarrow \operatorname{Der}_{M^{\prime}}(U), \quad X \longmapsto\left(\chi_{U}^{*}\right)^{-1} \circ X \circ \chi_{U}^{*},
$$

for all open $U \subseteq \widetilde{M^{\prime}}$. The pull-back of super-one-forms (denoted with a slight abuse of notation also by $\left.\chi_{U}^{*}\right) \chi_{U}^{*}: \Omega_{M^{\prime}}^{1}(U) \rightarrow \Omega_{M}^{1}\left(\tilde{\chi}^{-1}(U)\right)$ is then defined by the duality relations

$$
\left\langle X, \chi_{U}^{*}(\omega)\right\rangle:=\chi_{U}^{*}\left(\left\langle\chi_{* U}(X), \omega\right\rangle\right),
$$

for all $\omega \in \Omega_{M^{\prime}}^{1}(U)$ and $X \in \operatorname{Der}_{M}\left(\widetilde{\chi}^{-1}(U)\right)$. In the case where we only have that $\chi: M \rightarrow$ $\left.M^{\prime}\right|_{\widetilde{\chi}(\widetilde{M})}$ is a SMan-isomorphism we define the pull-back of super-one-forms by

$$
\chi_{U}^{*}(\omega):=\chi_{U \cap \widetilde{\chi}(\widetilde{M})}^{*}\left(\operatorname{res}_{U, U \cap \widetilde{\chi}(\widetilde{M})}(\omega)\right)
$$

for all $\omega \in \Omega_{M^{\prime}}^{1}(U)$ and all open $U \subseteq \widetilde{M^{\prime}}$. The following properties are immediate from this definition

$$
\chi_{U}^{*}(f \omega)=\chi_{U}^{*}(f) \chi_{U}^{*}(\omega), \quad \chi_{U}^{*}(\mathrm{~d} f)=\mathrm{d} \chi_{U}^{*}(f),
$$

for all $f \in \mathscr{O}_{M^{\prime}}(U)$ and $\omega \in \Omega_{M^{\prime}}^{1}(U)$. We shall denote the pull-back of global sections of $\Omega_{M^{\prime}}^{1}$ simply by $\chi^{*}:=\chi_{\widetilde{M}^{\prime}}^{*}: \Omega^{1}\left(M^{\prime}\right) \rightarrow \Omega^{1}(M)$.

In complete analogy to the case of ordinary manifolds, one can define a sheaf of differential graded superalgebras $\Omega_{M}^{\bullet}:=\Lambda^{\bullet} \Omega_{M}^{1}$, for which we shall use the same sign conventions as in DM99, §3.3]. Sections of this sheaf are called super-differential forms and we recall that for supermanifolds $M$ of dimension $n \mid m$ with $m \neq 0$ there are no top-degree forms.

Berezin integration: Given any object $M=\left(\widetilde{M}, \mathscr{O}_{M}\right)$ in SMan, one can construct the Berezinian sheaf $\operatorname{Ber}\left(\Omega_{M}^{1}\right)$ of the super-one-form sheaf $\Omega_{M}^{1}$. Notice that $\operatorname{Ber}\left(\Omega_{M}^{1}\right)$ is a sheaf of locally free left $\mathscr{O}_{M}$-supermodules: In local coordinates $\left(x^{1}, \ldots, x^{n+m}\right)=\left(x^{1}, \ldots, x^{n}, \theta^{1}, \ldots, \theta^{m}\right)$ of $M$ in $V$ an adapted basis for $\operatorname{Ber}\left(\Omega_{M}^{1}\right)(V)$ is given by $\left[\mathrm{d} x^{1}, \ldots, \mathrm{d} x^{n}, \mathrm{~d} \theta^{1}, \ldots, \mathrm{d} \theta^{m}\right]$. Given now any $f \in \mathscr{O}_{M}(V)$ with compact support in $V \subseteq \widetilde{M}$, we define the local Berezin integral over the open subsupermanifold $\left.M\right|_{V}$ by

$$
\int_{\left.M\right|_{V}}\left[\mathrm{~d} x^{1}, \ldots, \mathrm{d} x^{n}, \mathrm{~d} \theta^{1}, \ldots, \mathrm{d} \theta^{m}\right] f:=\int_{V} \mathrm{~d} x^{1} \cdots \mathrm{d} x^{n} f_{(1, \ldots, 1)},
$$

where we have used the expansion $f=\sum_{|I|<m} \theta^{I} f_{I}+\theta^{m} \cdots \theta^{1} f_{(1, \ldots, 1)}$ in terms of the odd coordinate functions. Here $I=\left(i_{1}, \ldots, i_{m}\right) \in\{0,1\}^{m}$ is a multiindex and $\theta^{I}:=\left(\theta^{m}\right)^{i_{1}} \ldots\left(\theta^{1}\right)^{i_{m}}$. Due to the change of variables formula, the local Berezin integral can be globalized, see e.g. [DM99, §3.10]. In the case of $M$ being oriented this yields a linear functional

$$
\int_{M}: \operatorname{Ber}\left(\Omega_{M}^{1}\right)_{\mathrm{c}}(\widetilde{M}) \longrightarrow \mathbb{R}, \quad v \longmapsto \int_{M} v
$$


on the compactly supported sections of $\operatorname{Ber}\left(\Omega_{M}^{1}\right)$, which for sections $v$ with support in $V$ is given by (2.22).

Given any SMan-isomorphism $\chi: M \rightarrow M^{\prime}$, there exists a pull-back $\chi^{*}: \operatorname{Ber}\left(\Omega_{M^{\prime}}^{1}\right) \rightarrow$ $\operatorname{Ber}\left(\Omega_{M}^{1}\right)$. In local coordinates $\left(x^{\prime 1}, \ldots, x^{\prime n+m}\right)$ of $M^{\prime}$ in $V^{\prime}$ and $\left(x^{1}, \ldots, x^{n+m}\right)$ of $M$ in $V=$ $\tilde{\chi}^{-1}\left(V^{\prime}\right)$, the pull-back is given by

$$
\begin{aligned}
\chi_{V^{\prime}}^{*}\left(\left[\mathrm{~d} x^{\prime 1}, \ldots, \mathrm{d} x^{\prime n+m}\right]\right) & =\left[\mathrm{d} \chi_{V^{\prime}}^{*}\left(x^{\prime 1}\right), \ldots, \mathrm{d} \chi_{V^{\prime}}^{*}\left(x^{\prime n+m}\right)\right] \\
& =\operatorname{Ber}(J(\chi))\left[\mathrm{d} x^{1}, \ldots, \mathrm{d} x^{n+m}\right],
\end{aligned}
$$

where $J(\chi)$ is the super-Jacobi matrix with entries defined by $\mathrm{d} \chi_{V^{\prime}}^{*}\left(x^{\prime i}\right)=\sum_{j=1}^{n+m} J(\chi)_{j}^{i} \mathrm{~d} x^{j}$. Notice the property $\chi_{U}^{*}(v f)=\chi_{U}^{*}(v) \chi_{U}^{*}(f)$, for all $v \in \operatorname{Ber}\left(\Omega_{M^{\prime}}^{1}\right)(U), f \in \mathscr{O}_{M^{\prime}}(U)$ and all open $U \subseteq \widetilde{M^{\prime}}$. Given now two oriented supermanifolds $M$ and $M^{\prime}$ together with an orientation preserving SMan-isomorphism $\chi: M \rightarrow M^{\prime}$, the global Berezin integral transforms as

$$
\int_{M} \chi^{*}(v)=\int_{M^{\prime}} v
$$

for all $v \in \operatorname{Ber}\left(\Omega_{M^{\prime}}^{1}\right)_{\mathrm{c}}\left(\widetilde{M^{\prime}}\right)$. This follows in the usual way from the aforementioned local change of variables formula.

\section{Super-Cartan supermanifolds}

We introduce the concept of super-Cartan structures on supermanifolds. These structures have their origin in the superspace formulation of supergravity, see e.g. WZ77. In our work they are required for constructing natural super-differential operators which govern the dynamics of super-field theories at the classical and quantum level. In this paper we shall only focus on the case where the super-principal bundle underlying the super-Cartan structure is globally trivial (and trivialized), which considerably simplifies our discussion as we do not have to deal with super-principal bundles and their associated super-vector bundles. Notice that the latter concepts are essentially well understood, see e.g. [BBHR91, but they are cumbersome to work with. To the best of our knowledge there is not yet a fully developed theory of (global) superCartan supermanifolds along the lines of standard treatments in ordinary differential geometry Sha97, Chapter 5]. In particular, we are not aware of conditions (on the basis supermanifold) which ensure the existence of super-Cartan structures or conditions which ensure that the underlying super-principal bundle is generically trivial. We hope to come back to this problem in a future work, which then would allow us to study super-field theories on globally non-trivial super-Cartan supermanifolds.

\subsection{Representation theoretic data and super-Poincaré super-Lie algebras}

We shall briefly review super-Poincaré super-Lie algebras in various dimensions. See e.g. Del99. and [Fre99, Lecture 3] for details.

Let $W$ be a finite-dimensional real vector space and $g: W \otimes W \rightarrow \mathbb{R}$ a Lorentz metric of signature $(+,-, \cdots,-)$. Let further $S$ be a real spin representation of the associated spin group $\operatorname{Spin}(W, g)$ and

$$
\Gamma: S \otimes S \longrightarrow W
$$

a symmetric and $\operatorname{Spin}(W, g)$-equivariant pairing. We denote the spin group actions on $W$ and $S$ by, respectively, $\rho^{W}: \operatorname{Spin}(W, g) \times W \rightarrow W$ and $\rho^{S}: \operatorname{Spin}(W, g) \times S \rightarrow S$. Moreover, we simply write $\mathfrak{s p i n}$ for the Lie algebra of $\operatorname{Spin}(W, g)$ and recall that the spin group actions above induce Lie algebra actions, which we denote by $\rho_{*}^{W}: \mathfrak{s p i n} \otimes W \rightarrow W$ and $\rho_{*}^{S}: \mathfrak{s p i n} \otimes S \rightarrow S$. 
Definition 3.1. Let us fix any choice of the data $(W, g, S, \Gamma)$.

(i) The super-Poincaré super-Lie algebra $\mathfrak{s p}$ (corresponding to this data) is given by the super-vector space

$$
\mathfrak{s p}:=(\mathfrak{s p i n} \oplus W) \oplus S
$$

(with $(\mathfrak{s p i n} \oplus W)$ even and $S$ odd), together with the super-Lie bracket defined by

$$
\begin{aligned}
& {\left[L_{1} \oplus w_{1} \oplus s_{1}, L_{2} \oplus w_{2} \oplus s_{2}\right]=} \\
& \quad\left[L_{1}, L_{2}\right] \oplus\left(\rho_{*}^{W}\left(L_{1} \otimes w_{2}-L_{2} \otimes w_{1}\right)-2 \Gamma\left(s_{1}, s_{2}\right)\right) \oplus \rho_{*}^{S}\left(L_{1} \otimes s_{2}-L_{2} \otimes s_{1}\right),
\end{aligned}
$$

for all $L_{1} \oplus w_{1} \oplus s_{1}, L_{2} \oplus w_{2} \oplus s_{2} \in \mathfrak{s p}$.

(ii) The supertranslation super-Lie algebra $\mathfrak{t}$ (corresponding to this data) is given by the super-vector space

$$
\mathfrak{t}:=W \oplus S
$$

(with $W$ even and $S$ odd), together with the super-Lie bracket defined by

$$
\left[w_{1} \oplus s_{1}, w_{2} \oplus s_{2}\right]=-2 \Gamma\left(s_{1}, s_{2}\right) \oplus 0,
$$

for all $w_{1} \oplus s_{1}, w_{2} \oplus s_{2} \in \mathfrak{t}$.

Remark 3.2. Notice that the bracket defined in (3.2b) is indeed a super-Lie bracket. The super-skew symmetry is evident from its definition and the super-Jacobi identity is a straightforward check using the $\operatorname{Spin}(W, g)$-equivariance of $\Gamma$, which implies that

$$
\rho_{*}^{W}\left(L \otimes \Gamma\left(s_{1}, s_{2}\right)\right)=\Gamma\left(\rho_{*}^{S}\left(L \otimes s_{1}\right), s_{2}\right)+\Gamma\left(s_{1}, \rho_{*}^{S}\left(L \otimes s_{2}\right)\right),
$$

for all $L \in \mathfrak{s p i n}$ and $s_{1}, s_{2} \in S$.

The following statement is easily shown. We therefore can omit the proof.

Proposition 3.3. For any choice of the data $(W, g, S, \Gamma)$, the super-Poincaré super-Lie algebra $\mathfrak{s p}$ is a super-Lie algebra extension of the Lie algebra $\mathfrak{s p i n}$ (regarded as a super-Lie algebra) by the supertranslation super-Lie algebra $\mathfrak{t}$, i.e.

$$
0 \longrightarrow \mathfrak{t} \longrightarrow \mathfrak{s p} \longrightarrow \mathfrak{s p i n} \longrightarrow 0
$$

with the obvious definition of the arrows, is a short exact sequence of super-Lie algebras.

The data $(W, g, S, \Gamma)$ we have introduced above is sufficient in order to construct the superPoincaré and supertranslation super-Lie algebras (corresponding to this choice of data). For our applications to super-Cartan supermanifolds and super-field theories we require some additional data. First, let us fix a positive cone $C \subset W$ of timelike vectors and assume that $\Gamma: S \otimes S \rightarrow W$ is positive in the sense that $\Gamma(s, s) \in \bar{C}$, for all $s \in S$, with $\Gamma(s, s)=0$ only for $s=0$. Here $\bar{C}$ denotes the closure of the cone $C \subset W$. The existence of such $\Gamma$ has been shown in Del99]. The positive cone $C \subset W$ will later play the role of a time-orientation. Next, we assume that we have given a $\operatorname{Spin}(W, g)$-invariant linear map

$$
\epsilon: S \otimes S \longrightarrow \mathbb{R}
$$

which is either a metric (of positive signature) or a symplectic structure. Such linear maps exist if $\operatorname{dim}(W)$ is not equal to 2 or 6 modulo 8, see DF99. Finally, we take as part of the data a choice of orientations $o_{W}$ of $W$ and $o_{S}$ of $S$. These orientations and also $\epsilon$ will be used define a canonical Berezinian density on any super-Cartan supermanifold and therefore a notation of integration. In summary, we will always assume as a starting point for our constructions that the data $\left(W, g, S, \Gamma, C, \epsilon, o_{W}, o_{S}\right)$ are given. 


\subsection{Super-Cartan structures}

In order to simplify our studies on super-Cartan supermanifolds we shall restrict our attention to super-Cartan structures which are based on globally trivial (and also trivialized) superprincipal $\operatorname{Spin}(W, g)$-bundles. Let us fix any choice of the data $\left(W, g, S, \Gamma, C, \epsilon, o_{W}, o_{S}\right)$.

Definition 3.4. Let $M$ be a $\operatorname{dim}(W) \mid \operatorname{dim}(S)$-dimensional supermanifold. A (globally trivial) super-Cartan structure on $M$ is a pair $(\Omega, E)$ consisting of an even super-one-form $\Omega \in$ $\Omega^{1}(M, \mathfrak{s p i n})$ (called the super-spin connection) and an even and non-degenerate super-one-form $E \in \Omega^{1}(M, \mathfrak{t})$ (called the supervielbein). The triple $\boldsymbol{M}:=(M, \Omega, E)$ is called a super-Cartan supermanifold.

Remark 3.5. Notice that the requirement that $E$ is non-degenerate fixes the dimension of $M$ to be the dimension $\operatorname{dim}(W) \mid \operatorname{dim}(S)$ of the supertranslation super-Lie algebra $\mathfrak{t}$.

To any super-Cartan supermanifold $\boldsymbol{M}=(M, \Omega, E)$ we can assign its supercurvature and supertorsion, which play an important role in supergravity. They are defined by

$$
\begin{aligned}
R_{M} & :=\mathrm{d} \Omega+[\Omega, \Omega] \in \Omega^{2}(M, \mathfrak{s p i n}), \\
T_{M} & :=\mathrm{d}_{\Omega} E:=\mathrm{d} E+[\Omega, E] \in \Omega^{2}(M, \mathfrak{t}),
\end{aligned}
$$

where the brackets are those induced by the super-Lie bracket in $\mathfrak{s p}$ via

$$
\begin{aligned}
{[\cdot, \cdot]: \Omega^{k}(M, \mathfrak{s p}) \otimes \Omega^{l}(M, \mathfrak{s p}) } & \longrightarrow \Omega^{k+l}(M, \mathfrak{s p}), \\
\left(\omega_{1} \otimes X_{1}\right) \otimes\left(\omega_{2} \otimes X_{2}\right) & \longmapsto(-1)^{\left|X_{1}\right|\left|\omega_{2}\right|} \omega_{1} \wedge \omega_{2} \otimes\left[X_{1}, X_{2}\right] .
\end{aligned}
$$

We now shall study integration on super-Cartan supermanifolds. Let us recall that the super-vector space $\mathfrak{t}$ has an adapted basis $\left\{p_{0}, \ldots, p_{\operatorname{dim}(M)-1}, q_{1}, \ldots, q_{\operatorname{dim}(S)}\right\}$, i.e. $p_{\alpha} \in W$ and $q_{a} \in S$, for all $\alpha=0, \ldots, \operatorname{dim}(M)-1$ and $a \in 1, \ldots, \operatorname{dim}(S)$. Making use of the Lorentz metric $g$ on $W$ and the metric (or symplectic structure) $\epsilon$ on $S$, we can demand that $\left\{p_{\alpha}\right\}$ is an orthonormal basis for $(W, g)$ and that $\left\{q_{a}\right\}$ is an orthonormal (or symplectic/Darboux) basis for $(S, \epsilon)$. Making further use of the orientations $o_{W}$ of $W$ and $o_{S}$ of $S$ we demand that these bases are oriented and finally by using the positive cone $C \subset W$ we demand that the basis for $W$ is time-oriented, i.e. the timelike basis vector $p_{0}$ lies in $C$. We shall call any adapted basis for $\mathfrak{t}$ which is of this kind an orthonormal (or orthosymplectic) time-oriented and oriented adapted basis for $\mathfrak{t}$. Notice that any two orthonormal (or orthosymplectic) timeoriented and oriented adapted bases for $\mathfrak{t}$ are related by a SVec-automorphism $L \in \mathrm{GL}(\mathfrak{t})$, whose block-matrix components (cf. (2.8) ) are $L_{1} \in \mathrm{SO}_{0}(1, \operatorname{dim}(W)-1), L_{2}=L_{3}=0$ and $L_{4} \in \mathrm{SO}(\operatorname{dim}(S))$ (or $L_{4} \in \operatorname{Sp}(\operatorname{dim}(S), \mathbb{R})$ ). Because of $\operatorname{det}\left(L_{1}\right)=\operatorname{det}\left(L_{4}\right)=1$ we find that $\operatorname{Ber}(L)=1$, cf. (2.9). We now may expand the supervielbein $E \in \Omega^{1}(M, \mathfrak{t})$ in terms of any orthonormal (or orthosymplectic) time-oriented and oriented adapted basis for $\mathfrak{t}$, which yields

$$
E=\sum_{\alpha=0}^{\operatorname{dim}(M)-1} e^{\alpha} \otimes p_{\alpha}+\sum_{a=1}^{\operatorname{dim}(S)} \xi^{a} \otimes q_{a},
$$

where all $e^{\alpha} \in \Omega^{1}(M)$ are even and all $\xi^{a} \in \Omega^{1}(M)$ are odd. Notice that the collection $\left\{e^{0}, \ldots, e^{\operatorname{dim}(W)-1}, \xi^{1}, \ldots, \xi^{\operatorname{dim}(S)}\right\}$ is an adapted basis for $\Omega^{1}(M)$ since $E$ was assumed to be non-degenerate. We hence can define an element of the Berezinian supermodule of $\Omega^{1}(M)$ by

$$
\operatorname{Ber}(E):=\left[e^{0}, \ldots, e^{\operatorname{dim}(M)-1}, \xi^{1}, \ldots, \xi^{\operatorname{dim}(S)}\right] \in \operatorname{Ber}\left(\Omega^{1}(M)\right) .
$$

Recalling (2.10), we find that this definition does not depend on the choice of the orthonormal (or orthosymplectic) time-oriented and oriented adapted basis for $\mathfrak{t}$, since, as we have explained above, any two such bases are related by an $L \in \mathrm{GL}(\mathfrak{t})$ with $\operatorname{Ber}(L)=1$. 
Using the Berezinian density (3.10) we can define a pairing on the compactly supported sections of the structure sheaf of $M$ by

$$
\langle\cdot, \cdot\rangle_{M}: \mathscr{O}_{\mathrm{c}}(M) \otimes \mathscr{O}_{\mathrm{c}}(M) \longrightarrow \mathbb{R}, \quad F_{1} \otimes F_{2} \longmapsto \int_{M} \operatorname{Ber}(E) F_{1} F_{2} .
$$

Notice that the $\mathbb{Z}_{2}$-parity of the linear map $\langle\cdot, \cdot\rangle_{M}$ is $\operatorname{dim}(S) \bmod 2$ and that $\langle\cdot, \cdot\rangle_{M}$ can be extended to all $F_{1}, F_{2} \in \mathscr{O}(M)$ with compactly overlapping support. Notice further that

$$
\left\langle F_{1}, F_{2}\right\rangle_{M}=(-1)^{\left|F_{1}\right|\left|F_{2}\right|}\left\langle F_{2}, F_{1}\right\rangle_{M}
$$

for all homogeneous $F_{1}, F_{2} \in \mathscr{O}(M)$ with compactly overlapping support.

We finish this subsection by defining a suitable category of super-Cartan supermanifolds.

Definition 3.6. The category SCart consists of the following objects and morphisms:

- The objects are all super-Cartan supermanifolds $\boldsymbol{M}=(M, \Omega, E)$.

- The morphisms $\chi: M \rightarrow M^{\prime}$ are all SMan-morphisms (denoted by the same symbol) $\chi: M \rightarrow M^{\prime}$, such that

1. $\tilde{\chi}: \widetilde{M} \rightarrow \widetilde{M^{\prime}}$ is an open embedding,

2. $\chi:\left.M \rightarrow M^{\prime}\right|_{\widetilde{\chi}(\widetilde{M})}$ is a SMan-isomorphism,

3. the super-Cartan structures are preserved, i.e. $\chi^{*}\left(\Omega^{\prime}\right)=\Omega$ and $\chi^{*}\left(E^{\prime}\right)=E$.

Remark 3.7. Since $\chi^{*}\left(E^{\prime}\right)=E$ it is clear that any SCart-morphism $\chi: M \rightarrow \boldsymbol{M}^{\prime}$ preserves the Berezinian densities (3.10), i.e.

$$
\chi_{\widetilde{\chi}(\widetilde{M})}^{*}\left(\operatorname{res}_{\widetilde{M^{\prime}}, \widetilde{\chi}(\widetilde{M})}\left(\operatorname{Ber}\left(E^{\prime}\right)\right)\right)=\operatorname{Ber}(E) .
$$

\subsection{Lorentz geometry on the reduced manifold}

Given any super-Cartan supermanifold $\boldsymbol{M}=(M, \Omega, E)$ we can equip the reduced manifold $\widetilde{M}$ with a Lorentz metric, an orientation and a time-orientation. Explicitly, the pull-back of the supervielbein $E \in \Omega^{1}(M, \mathfrak{t})$ along the embedding of the reduced manifold $\iota_{\widetilde{M}, M}: \widetilde{M} \rightarrow M$ provides us with a non-degenerate one-form on $\widetilde{M}$ with values in the even part $W$ of $\mathfrak{t}$ that we shall denote by $\widetilde{E}:=\iota_{\widetilde{M}, M}^{*}(E) \in \Omega^{1}(\widetilde{M}, W)$. We define a Lorentz metric $\widetilde{g}_{M}$ on $\widetilde{M}$ by setting

$$
\widetilde{g}_{M}: \Gamma^{\infty}(T \widetilde{M}) \times \Gamma^{\infty}(T \widetilde{M}) \longrightarrow C^{\infty}(\widetilde{M}), \quad(X, Y) \longmapsto g(\langle X, \widetilde{E}\rangle,\langle Y, \widetilde{E}\rangle)
$$

where $g: W \otimes W \rightarrow \mathbb{R}$ is the Lorentz metric on $W$ and $\langle\cdot, \cdot\rangle$ is the duality pairing between vector fields and one-forms on $\widetilde{M}$. The orientation $o_{W}$ of $W$ induces an orientation $\widetilde{o}_{M}$ of $\widetilde{M}$, which we may represent by the $\operatorname{volume}$ form $\operatorname{vol}(\widetilde{E}):=\widetilde{e}^{0} \wedge \cdots \wedge \widetilde{e}^{\operatorname{dim}(W)-1}$, where we have expanded $\widetilde{E}=\sum_{\alpha=0}^{\operatorname{dim}(W)-1} \widetilde{e}^{\alpha} \otimes p_{\alpha}$ in terms of any orthonormal time-oriented and oriented basis $\left\{p_{\alpha}\right\}$ for $W$. The positive cone $C \subset W$ of timelike vectors in $W$ induces a time-orientation $\widetilde{t}_{M}$ on the reduced oriented Lorentz manifold $\left(\widetilde{M}, \widetilde{g}_{M}, \widetilde{o}_{M}\right)$, which we may represent by the vector field $\widetilde{X}_{0}$ on $\widetilde{M}$ that is defined via the duality relations $\left\langle\widetilde{X}_{\alpha}, \widetilde{e}^{\beta}\right\rangle=\delta_{\alpha}^{\beta}$.

We shall now show that the assignment of the reduced oriented and time-oriented Lorentz manifolds $\widetilde{\boldsymbol{M}}:=\left(\widetilde{M}, \widetilde{g}_{\boldsymbol{M}}, \widetilde{o}_{\boldsymbol{M}}, \widetilde{t}_{\boldsymbol{M}}\right)$ to super-Cartan supermanifolds $\boldsymbol{M}$ is functorial. We define the category of oriented and time-oriented Lorentz manifolds otLor as follows: The objects are all oriented and time-oriented Lorentz manifolds and the morphisms are all open isometric embeddings that preserve the orientations and time-orientations. 
Proposition 3.8. The following assignment defines a functor $\sim$ : SCart $\rightarrow$ otLor: To any object $\boldsymbol{M}$ in SCart we assign the oriented and time-oriented Lorentz manifold $\widetilde{\boldsymbol{M}}:=\left(\widetilde{M}, \widetilde{g}_{\boldsymbol{M}}, \widetilde{o}_{\boldsymbol{M}}, \widetilde{t}_{\boldsymbol{M}}\right)$ that has been constructed above. To any morphism $\chi: M \rightarrow \boldsymbol{M}^{\prime}$ in SCart we assign the otLormorphism $\tilde{\chi}: \widetilde{\boldsymbol{M}} \rightarrow \widetilde{\boldsymbol{M}^{\prime}}$ that is defined by the reduced morphism $\tilde{\chi}: \widetilde{M} \rightarrow \widetilde{M^{\prime}}$.

Proof. We have to prove that the reduced morphism $\tilde{\chi}: \widetilde{M} \rightarrow \widetilde{M^{\prime}}$ is isometric and that it preserves the orientations and time-orientations. All these properties follow from the fact that $\widetilde{\chi}^{*}\left(\widetilde{E^{\prime}}\right)=\widetilde{E}$, which is shown by the calculation

$$
\begin{aligned}
\tilde{\chi}^{*}\left(\widetilde{E^{\prime}}\right) & =\widetilde{\chi}^{*} \circ \iota_{\widetilde{M}^{\prime}, M^{\prime}}^{*}\left(E^{\prime}\right)=\left(\iota_{\widetilde{M}^{\prime}, M^{\prime}} \circ \widetilde{\chi}\right)^{*}\left(E^{\prime}\right)=\left(\chi \circ \iota_{\widetilde{M}, M}\right)^{*}\left(E^{\prime}\right) \\
& =\iota_{\widetilde{M}, M}^{*} \circ \chi^{*}\left(E^{\prime}\right)=\iota_{\widetilde{M}, M}^{*}(E)=\widetilde{E},
\end{aligned}
$$

where in the third equality we have used the commutative diagram (2.13).

Due to this proposition we can define the chronological and causal future/past of a subset $A \subseteq \widetilde{M}$ in a super-Cartan supermanifold $M$ in terms of the corresponding reduced oriented and time-oriented Lorentz manifold $\widetilde{\boldsymbol{M}}$. (See e.g. [BGP07] for a definition of the chronological and causal future/past of a subset of a time-oriented Lorentz manifold.)

Definition 3.9. Let $M$ be a super-Cartan supermanifold and $A \subseteq \widetilde{M}$ a subset of its reduced manifold. The chronological future/past of $A$ in $M$ is defined by

$$
I_{M}^{ \pm}(A):=I_{\widetilde{M}}^{ \pm}(A) \subseteq \widetilde{M}
$$

and the causal future/past by

$$
J_{M}^{ \pm}(A):=J_{\widetilde{M}}^{ \pm}(A) \subseteq \widetilde{M} .
$$

We further define $I_{\boldsymbol{M}}(A):=I_{\boldsymbol{M}}^{+}(A) \cup I_{\boldsymbol{M}}^{-}(A)$ and $J_{\boldsymbol{M}}(A):=J_{\boldsymbol{M}}^{+}(A) \cup J_{\boldsymbol{M}}^{-}(A)$.

\subsection{The category of globally hyperbolic super-Cartan supermanifolds}

For our studies on super-field theories the category of globally hyperbolic super-Cartan supermanifolds will play a major role. It can be defined as a certain subcategory of SCart.

Definition 3.10. The category ghSCart consists of the following objects and morphisms:

- The objects in ghSCart are all objects $\boldsymbol{M}$ in SCart such that the reduced oriented and time-oriented Lorentz manifold $\widetilde{\boldsymbol{M}}$ is globally hyperbolic.

- The morphisms $\chi: M \rightarrow M^{\prime}$ between two objects $\boldsymbol{M}$ and $\boldsymbol{M}^{\prime}$ in ghSCart are all SCartmorphisms such that the image of the reduced morphism $\widetilde{\chi}: \widetilde{\boldsymbol{M}} \rightarrow \widetilde{\boldsymbol{M}^{\prime}}$ is a causally

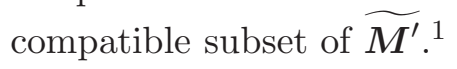

\section{Axiomatic definition of super-field theories}

Motivated by the examples we will discuss in Section 8, we shall give an axiomatic characterization of super-field theories by representation theoretic and geometric data. This is a reasonable and useful approach since all of our statements concerning the construction of super-QFTs in Section 5 can be made at this abstract level, so there is no need to focus on explicit models at this point. Moreover, the problem of constructing models of super-QFTs is thereby reduced to finding explicit realizations of the assumed representation theoretic and geometric data. It will be instructive to first provide some motivations explaining our choice of data.

\footnotetext{
${ }^{1}$ Recall that a subset $A \subseteq N$ of a time-oriented Lorentz manifold $N$ is called causally compatible provided that $J_{A}^{ \pm}(x)=J_{N}^{ \pm}(x) \cap A$, for all $x \in A$.
} 
Representation theoretic data: Motivated by Section 3, our first choice of data is given by an eight-tuple $\left(W, g, S, \Gamma, C, \epsilon, o_{W}, o_{S}\right)$ consisting of a finite-dimensional real vector space $W$, a Lorentz metric $g: W \otimes W \rightarrow \mathbb{R}$, a real spin representation $S$ of $\operatorname{Spin}(W, g)$, a symmetric and $\operatorname{Spin}(W, g)$-equivariant pairing $\Gamma: S \otimes S \rightarrow W$ which is positive with respect a choice of positive cone $C \subset W$ of timelike vectors, a $\operatorname{Spin}(W, g)$-invariant linear map $\epsilon: S \otimes S \rightarrow \mathbb{R}$ which is either a metric (of positive signature) or a symplectic structure, and orientations $o_{W}$ on $W$ and $o_{S}$ on $S$. It becomes evident from Section 3 that this data is required, on the one hand, to specify a super-Poincaré and supertranslation super-Lie algebra and, on the other hand, to describe super-Cartan structures on supermanifolds together with time-orientation and integration. In other words, the representation theoretic data fixes the local model space of the super-Cartan supermanifold and in particular its dimension to $\operatorname{dim}(W) \mid \operatorname{dim}(S)$. Physically speaking, this means that the representation theoretic data fixes the dimension of the reduced spacetime and the amount of supersymmetry.

Admissible super-Cartan supermanifolds: As we will show in Section 8 by studying explicit examples, one should not expect that the super-field theory will be defined on the whole category ghSCart of globally hyperbolic super-Cartan supermanifolds, see Definition 3.10. A common feature in many super-field theories (especially in supergravity) is that one has to impose constraints on the superfields in order to arrive at a reasonable theory. Such constraints may in particular include the supergravity supertorsion constraints [WZ77], which restrict the class of super-Cartan structures, i.e. the objects in ghSCart. As we would like to keep our axiomatic setting as flexible as possible, we shall at this point not specify the explicit form of these constraints but rather include a choice of full subcategory SLoc of ghSCart as a part of the data. Objects in SLoc will be called admissible super-Cartan supermanifolds and, at least for the examples studied in Section 8, a reasonable choice of SLoc is given by the supergravity supertorsion constraints.

Super-differential operators: We shall only consider super-field theories whose configurations on any object $\boldsymbol{M}=(M, \Omega, E)$ in SLoc can be described in terms of the super-vector space $\mathscr{O}(M):=\mathscr{O}(M)$ of global sections of the structure sheaf. Notice that $\mathscr{O}: \mathrm{SLoc}^{\mathrm{op}} \rightarrow \mathrm{SVec}$ is a functor, namely the global section functor. The dynamics of the super-field theory will be encoded in terms of super-differential operators $P_{\boldsymbol{M}}: \mathscr{O}(\boldsymbol{M}) \rightarrow \mathscr{O}(\boldsymbol{M})$ of $\mathbb{Z}_{2}$-parity $\operatorname{dim}(S) \bmod 2$, which are assumed to be natural in the sense that the diagram

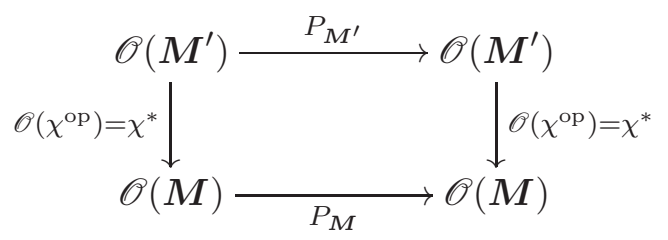

of linear maps commutes, for all SLoc ${ }^{\mathrm{op}}$-morphisms $\chi^{\mathrm{op}}: \boldsymbol{M}^{\prime} \rightarrow \boldsymbol{M}$ (i.e. all SLoc-morphisms $\left.\chi: \boldsymbol{M} \rightarrow \boldsymbol{M}^{\prime}\right) . P_{\boldsymbol{M}}$ can be regarded as the components of a natural transformation $P: \mathscr{O} \Rightarrow \mathscr{O}$, provided that we enlarge the morphism sets in SVec by parity reversing linear maps. More precisely, we shall replace the morphism sets $\operatorname{Hom}_{\mathrm{SVec}}\left(V, V^{\prime}\right)$ in $\mathrm{S} V_{e c}$ by the sets underlying the internal hom-objects $\underline{\operatorname{Hom}}\left(V, V^{\prime}\right)$. The corresponding category is then denoted by $\underline{\mathrm{SVec}}$ and we have an obvious functor $\mathrm{SVec} \rightarrow \underline{\mathrm{SVec}}$. Hence, $\mathscr{O}: \mathrm{SLoc}^{\mathrm{op}} \rightarrow \mathrm{SVec}$ defines a functor (denoted by the same symbol) $\mathscr{O}: \mathrm{SLoc}^{\mathrm{op}} \rightarrow \underline{\mathrm{SVec}} \underline{2}$ We additionally demand that, for any object $\boldsymbol{M}$

\footnotetext{
${ }^{2}$ Notice that enlarging the category SVec to $\underline{S V e c}$ is required only if $\operatorname{dim}(S)$ is odd, which is a peculiarity of the superparticle discussed in Section 8 In the more common situation where $\operatorname{dim}(S)$ is even, all our constructions can be done within the subcategory SVec of $\underline{\mathrm{SVec}}$, so there is no need to introduce the category $\underline{\mathrm{SVec}}$ in this case. We however decided to work with $\underline{\mathrm{SVec}}$ in order to develop a framework that is general enough to include the superparticle, which is a valuable example that can be analyzed in full detail, cf. Section 8 .
} 
in SLoc, the super-differential operator $P_{M}$ is formally super-self adjoint with respect to the pairing (3.11), i.e.

$$
\left\langle F_{1}, P_{M}\left(F_{2}\right)\right\rangle_{M}=(-1)^{\left|F_{1}\right|\left|P_{M}\right|}\left\langle P_{M}\left(F_{1}\right), F_{2}\right\rangle_{M},
$$

for all homogeneous $F_{1}, F_{2} \in \mathscr{O}(\boldsymbol{M})$ with compactly overlapping support. For many of our constructions we also have to assign retarded/advanced super-Green's operators to the superdifferential operators $P_{M}$, for all objects $M$ in SLoc. We therefore demand that, for any object $M$ in SLoc, the super-differential operator $P_{M}$ is super-Green's hyperbolic in the following sense:

Definition 4.1. Let $M$ be any object in SLoc. A homogeneous super-differential operator $P_{M}: \mathscr{O}(\boldsymbol{M}) \rightarrow \mathscr{O}(\boldsymbol{M})$ is called super-Green's hyperbolic if there exists a retarded and advanced super-Green's operator, i.e. linear maps $G_{\boldsymbol{M}}^{ \pm}: \mathscr{O}_{\mathrm{c}}(\boldsymbol{M}) \rightarrow \mathscr{O}(\boldsymbol{M})$ of $\mathbb{Z}_{2}$-parity $\left|G_{\boldsymbol{M}}^{ \pm}\right|=\left|P_{\boldsymbol{M}}\right|$ that satisfy

(i) $P_{M} \circ G_{M}^{ \pm}=\operatorname{id}_{\mathscr{O}_{\mathrm{c}}(\boldsymbol{M})}$,

(ii) $\left.G_{\boldsymbol{M}}^{ \pm} \circ P_{\boldsymbol{M}}\right|_{\mathscr{O}_{\mathrm{c}}(\boldsymbol{M})}=\operatorname{id}_{\mathscr{O}_{\mathrm{c}}(\boldsymbol{M})}$,

(iii) $\operatorname{supp}\left(G_{\boldsymbol{M}}^{ \pm}(F)\right) \subseteq J_{\boldsymbol{M}}^{ \pm}(\operatorname{supp}(F))$, for all $F \in \mathscr{O}_{\mathrm{c}}(\boldsymbol{M})$.

Motivated by the discussion above, we can now define abstractly a notion of super-field theories. Our present axiomatic framework is supposed to cover all super-field theories which in the physics literature would be called 'real superfields'. The typical examples in $1 \mid 1$ and 3|2-dimensions are discussed in Section 8, where it is shown that they comply with our axioms. In contrast, 'super-gauge theories' and 'chiral superfields' will require a more sophisticated set of axioms, which should include aspects of gauge invariance and the chirality constraints (see HS13 for an axiomatic approach to ordinary gauge theories). We shall leave these problems for future work and consider in the present work the case of (real) super-field theories which we characterize by the following axioms:

Definition 4.2. A super-field theory is specified by the following data:

1. A choice of the representation theoretic data $\left(W, g, S, \Gamma, C, \epsilon, o_{W}, o_{S}\right)$.

2. A full subcategory SLoc of ghSCart.

3. A natural transformation $P: \mathscr{O} \Rightarrow \mathscr{O}$ of functors from SLoc ${ }^{\mathrm{op}}$ to $\underline{\mathrm{SVec}}$, such that $P_{M}$ is a formally super-self adjoint and super-Green's hyperbolic super-differential operator of $\mathbb{Z}_{2}$-parity $\operatorname{dim}(S) \bmod 2$, for any object $\boldsymbol{M}$ in SLoc.

\section{Construction of super-quantum field theories}

We show that given any super-field theory according to Definition 4.2, one can construct a functor $\mathfrak{A}: S L o c \rightarrow S^{*}$ Alg which satisfies a supergeometric modification of the axioms of locally covariant quantum field theory BFV03. In other words, any super-field theory gives rise to a super-QFT. We establish a connection between the super-field theory and its associated super-QFT by showing that the latter has a locally covariant quantum field which satisfies (in a weak sense) the equations of motion given by the super-differential operators $P$. As usual our construction will be done in two steps. First, we assign to a super-field theory a functor $\mathfrak{L}: \operatorname{SLoc} \rightarrow \mathrm{X}$, where $\mathrm{X}$ is the category of super-symplectic spaces in the case of $\operatorname{dim}(S)$ even and the category of super-inner product spaces in the case of $\operatorname{dim}(S)$ odd. In the spirit of BFV03 this functor should be interpreted as a locally covariant classical field theory. The locally covariant classical field theory is then quantized by a quantization functor $\mathfrak{Q}: X \rightarrow \mathrm{S}^{*} \mathrm{Alg}$, which implements super-canonical commutation relations (SCCR) in the case of $\operatorname{dim}(S)$ even and super-canonical anticommutation relations (SCAR) in the case of $\operatorname{dim}(S)$ odd. 


\subsection{The functor $\mathscr{O}_{\mathrm{c}}: \mathrm{SLoc} \rightarrow \mathrm{SVec}$}

As a preparatory step, we shall show that assigning to objects $\boldsymbol{M}$ in SLoc the super-vector spaces $\mathscr{O}_{\mathrm{c}}(\boldsymbol{M}):=\mathscr{O}_{\mathrm{c}}(M)$ of compactly supported global sections of the structure sheaf can be described by a functor $\mathscr{O}_{\mathrm{c}}:$ SLoc $\rightarrow$ SVec. Given any SLoc-morphism $\chi: M \rightarrow \boldsymbol{M}^{\prime}$, we can define a SVec-morphism $\chi_{*}:=\mathscr{O}_{\mathrm{c}}(\chi): \mathscr{O}_{\mathrm{c}}(\boldsymbol{M}) \rightarrow \mathscr{O}_{\mathrm{c}}\left(\boldsymbol{M}^{\prime}\right)$, called the push-forward of compactly supported sections, by the following construction: Recall that for any SLoc-morphism $\chi: M \rightarrow M^{\prime}$ the corresponding SMan-morphism $\chi:\left.M \rightarrow M^{\prime}\right|_{\widetilde{\chi}(\widetilde{M})}$ is by assumption a SManisomorphism. In particular, we have a SVec-isomorphism

$$
\chi_{\widetilde{\chi}(\widetilde{M})}^{*}: \mathscr{O}_{M^{\prime}}(\widetilde{\chi}(\widetilde{M})) \longrightarrow \mathscr{O}_{M}(\widetilde{M})
$$

which induces a SVec-isomorphism on compactly supported sections

$$
\left(\chi_{\widetilde{\chi}(\widetilde{M})}^{*}\right)^{-1}: \mathscr{O}_{M, \mathrm{c}}(\widetilde{M}) \longrightarrow \mathscr{O}_{M^{\prime}, \mathrm{c}}(\widetilde{\chi}(\widetilde{M})) .
$$

Making use of the sheaf properties of $\mathscr{O}_{M^{\prime}}$, we can define a SVec-morphism

$$
\operatorname{ext}_{\widetilde{\chi}(\widetilde{M}), \widetilde{M^{\prime}}}: \mathscr{O}_{M^{\prime}, \mathrm{c}}(\widetilde{\chi}(\widetilde{M})) \longrightarrow \mathscr{O}_{M^{\prime}, \mathrm{c}}\left(\widetilde{M^{\prime}}\right)
$$

which extends compactly supported sections by zero. This SVec-morphism is a monomorphism since

$$
\operatorname{res}_{\widetilde{M^{\prime}}, \widetilde{\chi}(\widetilde{M})} \circ \operatorname{ext}_{\widetilde{\chi}(\widetilde{M}), \widetilde{M^{\prime}}}=\operatorname{id}_{\mathscr{O}_{M^{\prime}, \mathrm{c}}(\widetilde{\chi}(\widetilde{M}))}
$$

We define the push-forward of compactly supported sections by the composition

$$
\mathscr{O}_{\mathrm{c}}(\chi):=\chi_{*}:=\operatorname{ext}_{\widetilde{\chi}(\widetilde{M}), \widetilde{M^{\prime}}} \circ\left(\chi_{\widetilde{\chi}(\widetilde{M})}^{*}\right)^{-1}: \mathscr{O}_{\mathrm{c}}(\boldsymbol{M}) \longrightarrow \mathscr{O}_{\mathrm{c}}\left(\boldsymbol{M}^{\prime}\right)
$$

and notice that it is a SVec-monomorphism. The following lemma collects important properties of the push-forward of compactly supported sections, which shall be frequently used in our work.

Lemma 5.1. Let $\chi: M \rightarrow M^{\prime}$ be any SLoc-morphism. Then the following properties hold true:

(i) $\chi^{*} \circ \chi_{*}=\operatorname{id}_{\mathscr{O}_{\mathrm{c}}(\boldsymbol{M})}$ and $\chi_{*} \circ \chi^{*}(F)=F$, for all $F \in \mathscr{O}_{\mathrm{c}}\left(\boldsymbol{M}^{\prime}\right)$ such that $\operatorname{supp}(F) \subseteq \widetilde{\chi}(\widetilde{M})$.

(ii) $\left\langle F_{1}, \chi_{*}\left(F_{2}\right)\right\rangle_{\boldsymbol{M}^{\prime}}=\left\langle\chi^{*}\left(F_{1}\right), F_{2}\right\rangle_{\boldsymbol{M}}$, for all $F_{1} \in \mathscr{O}\left(\boldsymbol{M}^{\prime}\right)$ and $F_{2} \in \mathscr{O}_{\mathrm{c}}(\boldsymbol{M})$, where the pairing is defined in (3.11).

(iii) $\operatorname{id}_{\boldsymbol{M}_{*}}=\operatorname{id}_{\mathscr{O}_{\mathrm{c}}(\boldsymbol{M})}$ and $\left(\chi^{\prime} \circ \chi\right)_{*}=\chi_{*}^{\prime} \circ \chi_{*}$, for all SLoc-morphisms $\chi^{\prime}: \boldsymbol{M}^{\prime} \rightarrow \boldsymbol{M}^{\prime \prime}$.

Proof. Item (i) is shown by two simple calculations. The first part follows from

$$
\chi^{*} \circ \chi_{*}=\chi_{\widetilde{\chi}(\widetilde{M})}^{*} \circ \operatorname{res}_{\widetilde{M^{\prime}}, \widetilde{\chi}(\widetilde{M})} \circ \operatorname{ext}_{\widetilde{\chi}(\widetilde{M}), \widetilde{M^{\prime}}} \circ\left(\chi_{\widetilde{\chi}(\widetilde{M})}^{*}\right)^{-1}=\operatorname{id}_{\operatorname{id}_{\mathscr{O}_{C}(M)}},
$$

where in the first equality we have used the diagram (2.12) characterizing sheaf morphisms (applied to $U=\widetilde{M^{\prime}}$ and $V=\widetilde{\chi}(\widetilde{M})$ ) and in the second equality we have used (5.4). To show the second part, notice that if $F \in \mathscr{O}_{\mathrm{c}}\left(\boldsymbol{M}^{\prime}\right)$ is such that $\operatorname{supp}(F) \subseteq \widetilde{\chi}(\widetilde{M})$, then $\chi^{*}(F) \in \mathscr{O}_{\mathrm{c}}(\boldsymbol{M})$, so the composition $\chi_{*} \circ \chi^{*}(F)$ is well-defined. By using the same argument as above we find that

$$
\begin{aligned}
\chi_{*} \circ \chi^{*}(F) & =\operatorname{ext}_{\widetilde{\chi}(\widetilde{M}), \widetilde{M^{\prime}}} \circ\left(\chi_{\widetilde{\chi}(\widetilde{M})}^{*}\right)^{-1} \circ \chi_{\widetilde{\chi}(\widetilde{M})}^{*} \circ \operatorname{res}_{\widetilde{M^{\prime}}, \widetilde{\chi}(\widetilde{M})}(F) \\
& =\operatorname{ext}_{\widetilde{\chi}(\widetilde{M}), \widetilde{M^{\prime}}} \circ \operatorname{res}_{\widetilde{M^{\prime}}, \widetilde{\chi}(\widetilde{M})}(F)=F,
\end{aligned}
$$


where the last equality holds true by direct inspection.

Item (ii) holds true as a consequence of the transformation formula for the Berezin integral (2.25) and the property (3.13); explicitly, we have

$$
\begin{aligned}
\left\langle F_{1}, \chi_{*}\left(F_{2}\right)\right\rangle_{M^{\prime}} & =\int_{M^{\prime}} \operatorname{Ber}\left(E^{\prime}\right) F_{1} \chi_{*}\left(F_{2}\right) \\
& =\int_{M^{\prime} \mid} \operatorname{res}_{\left.\widetilde{M^{\prime}}, \widetilde{\chi}\right)}(\widetilde{M}) \\
& =\int_{M} \operatorname{Ber}(E) \chi_{\widetilde{\chi}(\widetilde{M})}^{*}\left(\operatorname{res}_{\widetilde{M^{\prime}}, \widetilde{\chi}(\widetilde{M})}\left(F_{1}\right)\right) \chi_{\widetilde{\chi}(\widetilde{M})}^{*}\left(\operatorname{res}_{\widetilde{M^{\prime}}, \widetilde{\chi}(\widetilde{M})}\left(\chi_{*}\left(F_{2}\right)\right)\right) \\
& =\int_{M} \operatorname{Ber}(E) \chi^{*}\left(F_{1}\right) \chi^{*}\left(\chi_{*}\left(F_{2}\right)\right)=\left\langle\chi^{*}\left(F_{1}\right), F_{2}\right\rangle_{M},
\end{aligned}
$$

for all $F_{1} \in \mathscr{O}\left(\boldsymbol{M}^{\prime}\right)$ and $F_{2} \in \mathscr{O}_{\mathrm{c}}(\boldsymbol{M})$. In the second equality we have used that the support of $\chi_{*}\left(F_{2}\right)$ is contained in $\widetilde{\chi}(\widetilde{M})$ and in the last equality item (i) of the present lemma.

The first part of item (iii) follows immediately from the definition (5.5) and the second part from the following calculation

$$
\begin{aligned}
& \left(\chi^{\prime} \circ \chi\right)_{*}=\operatorname{ext}_{\tilde{\chi}^{\prime}(\widetilde{\chi}(\widetilde{M})), \widetilde{M^{\prime \prime}}} \circ\left(\left(\chi^{\prime} \circ \chi\right)_{\tilde{\chi}^{\prime}(\widetilde{\chi}(\widetilde{M}))}^{*}\right)^{-1} \\
& =\operatorname{ext}_{\tilde{\chi}^{\prime}(\widetilde{\chi}(\widetilde{M})), \widetilde{M^{\prime \prime}}} \circ\left(\chi_{\widetilde{\chi}(\widetilde{M})}^{*} \circ \chi_{\tilde{\chi}^{\prime}(\widetilde{\chi}(\widetilde{M}))}^{\prime *}\right)^{-1}
\end{aligned}
$$

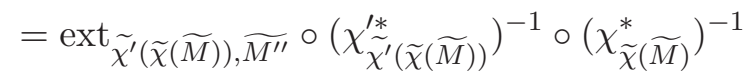

$$
\begin{aligned}
& =\operatorname{ext}_{\tilde{\chi}^{\prime}(\widetilde{\chi}(\widetilde{M})), \widetilde{M^{\prime \prime}}} \circ\left(\chi_{\tilde{\chi}^{\prime}(\widetilde{\chi}(\widetilde{M}))}^{\prime *}\right)^{-1} \circ \operatorname{res}_{\widetilde{M^{\prime}}, \widetilde{\chi}(\widetilde{M})} \circ \operatorname{ext}_{\widetilde{\chi}(\widetilde{M}), \widetilde{M^{\prime}}} \circ\left(\chi_{\widetilde{\chi}(\widetilde{M})}^{*}\right)^{-1}
\end{aligned}
$$

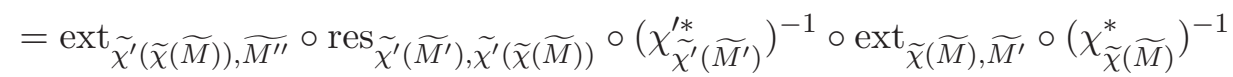

$$
\begin{aligned}
& =\operatorname{ext}_{{\widetilde{\chi^{\prime}}}^{\prime}\left(\widetilde{M^{\prime}}\right), \widetilde{M^{\prime \prime}}} \circ\left(\chi_{\widetilde{\chi}^{\prime}\left(\widetilde{M^{\prime}}\right)}^{\prime *}\right)^{-1} \circ \operatorname{ext}_{\widetilde{\chi}(\widetilde{M}), \widetilde{M^{\prime}}} \circ\left(\chi_{\widetilde{\chi}(\widetilde{M})}^{*}\right)^{-1} \\
& =\chi_{*}^{\prime} \circ \chi_{*},
\end{aligned}
$$

where we have made frequent use of standard properties of sheaf morphisms.

Corollary 5.2. The following assignment is a functor $\mathscr{O}_{\mathrm{c}}:$ SLoc $\rightarrow \mathrm{SVec}:$ To any object $\boldsymbol{M}$ in SLoc we assign the super-vector space $\mathscr{O}_{\mathrm{c}}(\boldsymbol{M}):=\mathscr{O}_{\mathrm{c}}(M)$ and to any SLoc-morphism $\chi: \boldsymbol{M} \rightarrow \boldsymbol{M}^{\prime}$ we assign the push-forward $\mathscr{O}_{\mathrm{c}}(\chi):=\chi_{*}: \mathscr{O}_{\mathrm{c}}(\boldsymbol{M}) \rightarrow \mathscr{O}_{\mathrm{c}}\left(\boldsymbol{M}^{\prime}\right)$ defined in (5.5).

Proof. This is a direct consequence of Lemma 5.1 (iii).

\subsection{Properties of the super-Green's operators}

Let us fix any super-field theory according to Definition 4.2. By assumption, there exists a retarded and advanced super-Green's operator $G_{\boldsymbol{M}}^{ \pm}: \mathscr{O}_{\mathrm{c}}(\boldsymbol{M}) \rightarrow \mathscr{O}(\boldsymbol{M})$ for the super-differential operator $P_{\boldsymbol{M}}: \mathscr{O}(\boldsymbol{M}) \rightarrow \mathscr{O}(\boldsymbol{M})$, for all objects $\boldsymbol{M}$ in SLoc. We shall now derive important properties of the super-Green's operators.

Lemma 5.3. Let $M$ be any object in SLoc. Then

$$
\left\langle F_{1}, G_{M}^{ \pm}\left(F_{2}\right)\right\rangle_{M}=(-1)^{\left(\left|F_{1}\right|+\left|P_{M}\right|\right)\left|P_{M}\right|}\left\langle G_{M}^{\mp}\left(F_{1}\right), F_{2}\right\rangle_{M},
$$

for all homogeneous $F_{1}, F_{2} \in \mathscr{O}_{\mathrm{c}}(\boldsymbol{M})$. 
Proof. The proof follows from a short calculation

$$
\begin{aligned}
\left\langle F_{1}, G_{M}^{ \pm}\left(F_{2}\right)\right\rangle_{M} & =\left\langle P_{M} \circ G_{M}^{\mp}\left(F_{1}\right), G_{M}^{ \pm}\left(F_{2}\right)\right\rangle_{M} \\
& =(-1)^{\left(\left|F_{1}\right|+\left|G_{M}^{\mp}\right|\right)\left|P_{M}\right|}\left\langle G_{M}^{\mp}\left(F_{1}\right), P_{M} \circ G_{M}^{ \pm}\left(F_{2}\right)\right\rangle_{M} \\
& =(-1)^{\left(\left|F_{1}\right|+\left|P_{M}\right|\right)\left|P_{M}\right|}\left\langle G_{\boldsymbol{M}}^{\mp}\left(F_{1}\right), F_{2}\right\rangle_{\boldsymbol{M}} .
\end{aligned}
$$

The first equality holds because of property (i) of Definition 4.1. The integral on the right-hand side is well-defined because of property (iii) of the same Definition and the fact that the reduced Lorentz manifold $\widetilde{M}$ is by assumption globally hyperbolic. The second equality follows from formal super-self adjointness of $P_{M}$, cf. (4.2). The last equality is a consequence of property (i) of Definition 4.1 and $\left|G_{M}^{\mp}\right|=\left|P_{M}\right|$.

We define the retarded-minus-advanced super-Green's operator

$$
G_{M}:=G_{M}^{+}-G_{M}^{-}: \mathscr{O}_{\mathrm{c}}(\boldsymbol{M}) \longrightarrow \mathscr{O}_{\mathrm{sc}}(\boldsymbol{M}) \subseteq \mathscr{O}(\boldsymbol{M}),
$$

whose image lies in the super-vector space $\mathscr{O}_{\mathrm{sc}}(\boldsymbol{M})$ of spacelike compact sections (see BGP07, Notation 3.4.5]) of the structure sheaf because of Definition 4.1 (iii). This operator has the following properties.

Theorem 5.4. Let $\boldsymbol{M}$ be any object in SLoc. Then the sequence of linear maps

$$
0 \longrightarrow \mathscr{O}_{\mathrm{c}}(\boldsymbol{M}) \stackrel{P_{M}}{\longrightarrow} \mathscr{O}_{\mathrm{c}}(\boldsymbol{M}) \stackrel{G_{M}}{\longrightarrow} \mathscr{O}_{\mathrm{sc}}(\boldsymbol{M}) \stackrel{P_{M}}{\longrightarrow} \mathscr{O}_{\mathrm{sc}}(\boldsymbol{M})
$$

is a complex which is exact everywhere.

Proof. The proof follows easily by adapting the steps in the proofs of [BG11, Theorem 3.5] or [BGP07, Theorem 3.4.7] to our supergeometric setting. We therefore can omit the details.

Corollary 5.5. Let $M$ be any object in SLoc. Then the retarded and advanced super-Green's operators $G_{M}^{ \pm}$for $P_{M}$ are unique.

Proof. Let us assume that there are two retarded/advanced super-Green's operators $G_{M}^{ \pm}$and $\bar{G}_{\boldsymbol{M}}^{ \pm}$for $P_{\boldsymbol{M}}$. Then, for any $F \in \mathscr{O}_{\mathrm{c}}(\boldsymbol{M})$, we have that $\Phi:=G_{\boldsymbol{M}}^{ \pm}(F)-\bar{G}_{\boldsymbol{M}}^{ \pm}(F)$ has $\operatorname{supp}(\Phi) \subseteq$ $J_{M}^{ \pm}(K)$, for some compact $K \subseteq \widetilde{M}$, and satisfies $P_{\boldsymbol{M}}(\Phi)=0$. We now show that $\Phi=0$ and hence that $G_{\boldsymbol{M}}^{ \pm}=\bar{G}_{\boldsymbol{M}}^{ \pm}$as $F \in \mathscr{O}_{\mathrm{c}}(\boldsymbol{M})$ was arbitrary. Indeed, we have that

$$
\left\langle F^{\prime}, \Phi\right\rangle_{M}=\left\langle P_{M} \circ G_{M}^{\mp}\left(F^{\prime}\right), \Phi\right\rangle_{M}=(-1)^{\left(\left|F^{\prime}\right|+\left|G_{M}^{\mp}\right|\right)\left|P_{M}\right|}\left\langle G_{M}^{\mp}\left(F^{\prime}\right), P_{M}(\Phi)\right\rangle_{M}=0,
$$

for all $F^{\prime} \in \mathscr{O}_{\mathrm{c}}(\boldsymbol{M})$, which implies that $\Phi=0$.

We shall now show that the retarded/advanced super-Green's operators are natural in the following sense.

Lemma 5.6. Let $\chi: M \rightarrow M^{\prime}$ be any morphism in SLoc. Then

$$
G_{M}^{ \pm}=\chi^{*} \circ G_{M^{\prime}}^{ \pm} \circ \chi_{*}
$$

as linear maps from $\mathscr{O}_{\mathrm{c}}(\boldsymbol{M})$ to $\mathscr{O}(\boldsymbol{M})$.

Proof. Let us define $\bar{G}_{M}^{ \pm}:=\chi^{*} \circ G_{M^{\prime}}^{ \pm} \circ \chi_{*}$. We will show that $\bar{G}_{M}^{ \pm}$is a retarded/advanced super-Green's operator for $P_{M}$, which due to the uniqueness result in Corollary 5.5 implies that $\bar{G}_{\boldsymbol{M}}^{ \pm}=G_{\boldsymbol{M}}^{ \pm}$. 
We have to show that $\bar{G}_{M}^{ \pm}$satisfies the three conditions of Definition 4.1. Item (i) is satisfied because of

$$
P_{\boldsymbol{M}} \circ \bar{G}_{\boldsymbol{M}}^{ \pm}=P_{\boldsymbol{M}} \circ \chi^{*} \circ G_{\boldsymbol{M}^{\prime}}^{ \pm} \circ \chi_{*}=\chi^{*} \circ P_{\boldsymbol{M}^{\prime}} \circ G_{\boldsymbol{M}^{\prime}}^{ \pm} \circ \chi_{*}=\chi^{*} \circ \chi_{*}=\operatorname{id}_{\mathscr{O}_{\mathrm{c}}(\boldsymbol{M})},
$$

where in the second equality we have used (4.1) and in the last equality Lemma 5.1 (i). Item (ii) is satisfied because of

$$
\begin{aligned}
\left.\bar{G}_{\boldsymbol{M}}^{ \pm} \circ P_{\boldsymbol{M}}\right|_{\mathscr{O}_{\mathrm{c}}(\boldsymbol{M})} & =\chi^{*} \circ G_{\boldsymbol{M}^{\prime}}^{ \pm} \circ \chi_{*} \circ P_{\boldsymbol{M}} \circ \chi^{*} \circ \chi_{*}=\chi^{*} \circ G_{\boldsymbol{M}^{\prime}}^{ \pm} \circ \chi_{*} \circ \chi^{*} \circ P_{\boldsymbol{M}^{\prime}} \circ \chi_{*} \\
& =\chi^{*} \circ G_{\boldsymbol{M}^{\prime}}^{ \pm} \circ P_{\boldsymbol{M}^{\prime}} \circ \chi_{*}=\chi^{*} \circ \chi_{*}=\operatorname{id}_{\mathscr{O}_{\mathrm{c}}(\boldsymbol{M})} .
\end{aligned}
$$

In the second equality we have used (4.1) and in the first, third and last equality Lemma 5.1 (i). To show that item (iii) is satisfied we use the same argument as in [BG11, Lemma 3.2], which is based on the causal compatibility of the image of the reduced morphism $\tilde{\chi}: \widetilde{M} \rightarrow \widetilde{M^{\prime}}$. Indeed,

$$
\begin{aligned}
\operatorname{supp}\left(\bar{G}_{\boldsymbol{M}}^{ \pm}(F)\right) & =\operatorname{supp}\left(\chi^{*} \circ G_{\boldsymbol{M}^{\prime}}^{ \pm} \circ \chi_{*}(F)\right) \subseteq \widetilde{\chi}^{-1}\left(\operatorname{supp}\left(G_{\boldsymbol{M}^{\prime}}^{ \pm} \circ \chi_{*}(F)\right)\right) \\
& \subseteq \widetilde{\chi}^{-1}\left(J_{\boldsymbol{M}^{\prime}}^{ \pm}(\widetilde{\chi}(\operatorname{supp}(F)))\right)=J_{\boldsymbol{M}}^{ \pm}(\operatorname{supp}(F))
\end{aligned}
$$

for all $F \in \mathscr{O}_{\mathrm{c}}(\boldsymbol{M})$.

\subsection{The functor $\mathfrak{L}: \mathrm{SLoc} \rightarrow \mathrm{X}$}

Let us fix any super-field theory according to Definition 4.2. For any object $\boldsymbol{M}$ in SLoc we can define a linear map

$$
\tau_{\boldsymbol{M}}: \mathscr{O}_{\mathrm{c}}(\boldsymbol{M}) \otimes \mathscr{O}_{\mathrm{c}}(\boldsymbol{M}) \longrightarrow \mathbb{R}, \quad F_{1} \otimes F_{2} \longmapsto \tau_{\boldsymbol{M}}\left(F_{1}, F_{2}\right)=\left\langle G_{\boldsymbol{M}}\left(F_{1}\right), F_{2}\right\rangle_{\boldsymbol{M}},
$$

where $G_{M}:=G_{M}^{+}-G_{M}^{-}$is the retarded-minus-advanced super-Green's operator and $\langle\cdot, \cdot\rangle_{M}$ is the pairing (3.11). Since, by definition, the $\mathbb{Z}_{2}$-parity of $G_{M}$ agrees with that of the pairing, the linear map $\tau_{M}$ is even and hence a SVec-morphism. As a consequence of (3.12) and Lemma 5.3 we find that

$$
\begin{aligned}
\tau_{M}\left(F_{1}, F_{2}\right) & =(-1)^{\left|P_{M}\right|+1}(-1)^{\left|F_{1}\right|\left|F_{2}\right|} \tau_{\boldsymbol{M}}\left(F_{2}, F_{1}\right) \\
& = \begin{cases}-(-1)^{\left|F_{1}\right|\left|F_{2}\right|} \tau_{\boldsymbol{M}}\left(F_{2}, F_{1}\right) & , \text { for } \operatorname{dim}(S) \text { even }, \\
(-1)^{\left|F_{1}\right|\left|F_{2}\right|} \tau_{\boldsymbol{M}}\left(F_{2}, F_{1}\right) & , \text { for } \operatorname{dim}(S) \text { odd },\end{cases}
\end{aligned}
$$

for all homogeneous $F_{1}, F_{2} \in \mathscr{O}_{\mathrm{c}}(\boldsymbol{M})$. Hence, $\tau_{\boldsymbol{M}}$ is super-skew symmetric if $\operatorname{dim}(S)$ is even and super-symmetric if $\operatorname{dim}(S)$ is odd.

Let us recall that by Theorem 5.4 the kernel of the linear map $G_{\boldsymbol{M}}: \mathscr{O}_{\mathrm{c}}(\boldsymbol{M}) \rightarrow \mathscr{O}_{\mathrm{sc}}(\boldsymbol{M})$ coincides with the image of $P_{\boldsymbol{M}}: \mathscr{O}_{\mathrm{c}}(\boldsymbol{M}) \rightarrow \mathscr{O}_{\mathrm{c}}(\boldsymbol{M})$. As a consequence, the SVec-morphism $\tau_{M}$ defined in (5.19) descends to the SVec-morphism (denoted with a slight abuse of notation by the same symbol)

$$
\tau_{\boldsymbol{M}}: \frac{\mathscr{O}_{\mathrm{c}}(\boldsymbol{M})}{P_{\boldsymbol{M}}\left(\mathscr{O}_{\mathrm{c}}(\boldsymbol{M})\right)} \otimes \frac{\mathscr{O}_{\mathrm{c}}(\boldsymbol{M})}{P_{\boldsymbol{M}}\left(\mathscr{O}_{\mathrm{c}}(\boldsymbol{M})\right)} \longrightarrow \mathbb{R}, \quad\left[F_{1}\right] \otimes\left[F_{2}\right] \longmapsto\left\langle G_{\boldsymbol{M}}\left(F_{1}\right), F_{2}\right\rangle_{\boldsymbol{M}},
$$

which is weakly non-degenerate, i.e. $\tau_{\boldsymbol{M}}\left(\left[F_{1}\right],\left[F_{2}\right]\right)=0$ for all $\left[F_{1}\right] \in \mathscr{O}_{\mathrm{c}}(\boldsymbol{M}) / P_{\boldsymbol{M}}\left(\mathscr{O}_{\mathrm{c}}(\boldsymbol{M})\right)$ implies that $\left[F_{2}\right]=0$. The pair

$$
\mathfrak{L}(\boldsymbol{M}):=\left(\frac{\mathscr{O}_{\mathrm{c}}(\boldsymbol{M})}{P_{\boldsymbol{M}}\left(\mathscr{O}_{\mathrm{c}}(\boldsymbol{M})\right)}, \tau_{\boldsymbol{M}}\right)
$$

is therefore a super-symplectic space if $\operatorname{dim}(S)$ is even and a super-inner product space if $\operatorname{dim}(S)$ is odd.

We shall now show that the assignment (5.22) is functorial. For this we introduce the following category which depends on the choice of super-field theory via $\operatorname{dim}(S) \bmod 2$. 
Definition 5.7. The category $X$ consists of the following objects and morphisms:

- The objects are all pairs $\boldsymbol{V}:=(V, \tau)$ consisting of a real super-vector space $V$ and a weakly non-degenerate SVec-morphism $\tau: V \otimes V \rightarrow \mathbb{R}$, which is super-skew symmetric if $\operatorname{dim}(S)$ is even and super-symmetric if $\operatorname{dim}(S)$ is odd, i.e.

$$
\tau\left(v_{1}, v_{2}\right)=(-1)^{\operatorname{dim}(S)+1}(-1)^{\left|v_{1}\right|\left|v_{2}\right|} \tau\left(v_{2}, v_{1}\right),
$$

for all homogeneous $v_{1}, v_{2} \in V$.

- The morphisms $L: \boldsymbol{V} \rightarrow \boldsymbol{V}^{\prime}$ are all SVec-morphisms (denoted by the same symbol) $L: V \rightarrow V^{\prime}$ satisfying $\tau^{\prime} \circ(L \otimes L)=\tau$.

Proposition 5.8. The following assignment is a functor $\mathfrak{L}: \mathrm{SLoc} \rightarrow \mathrm{X}:$ To any object $M$ in SLoc we assign the object $\mathfrak{L}(\boldsymbol{M})$ in $\mathrm{X}$ given by (5.22) and to any SLoc-morphism $\chi: \boldsymbol{M} \rightarrow \boldsymbol{M}^{\prime}$ we assign the $\mathbf{X}$-morphism

$$
\mathfrak{L}(\chi): \mathfrak{L}(\boldsymbol{M}) \longrightarrow \mathfrak{L}\left(\boldsymbol{M}^{\prime}\right), \quad[F] \longmapsto\left[\chi_{*}(F)\right] .
$$

Proof. We have already seen above that $\mathfrak{L}(\boldsymbol{M})$ is an object in X. It remains to show that (5.24) is well-defined and an X-morphism. It is well-defined since

$$
\begin{aligned}
\chi_{*} \circ P_{\boldsymbol{M}}(F) & =\chi_{*} \circ P_{\boldsymbol{M}} \circ \chi^{*} \circ \chi_{*}(F)=\chi_{*} \circ \chi^{*} \circ P_{\boldsymbol{M}^{\prime}} \circ \chi_{*}(F) \\
& =P_{\boldsymbol{M}^{\prime}} \circ \chi_{*}(F) \in P_{\boldsymbol{M}^{\prime}}\left(\mathscr{O}_{\mathbf{C}}\left(\boldsymbol{M}^{\prime}\right)\right),
\end{aligned}
$$

for all $F \in \mathscr{O}_{\mathrm{c}}(\boldsymbol{M})$. Moreover it is an $\mathrm{X}$-morphism since

$$
\begin{aligned}
\tau_{\boldsymbol{M}^{\prime}}\left(\left[\chi_{*}\left(F_{1}\right)\right],\left[\chi_{*}\left(F_{2}\right)\right]\right) & =\left\langle G_{\boldsymbol{M}^{\prime}} \circ \chi_{*}\left(F_{1}\right), \chi_{*}\left(F_{2}\right)\right\rangle_{\boldsymbol{M}^{\prime}}=\left\langle\chi^{*} \circ G_{\boldsymbol{M}^{\prime}} \circ \chi_{*}\left(F_{1}\right), F_{2}\right\rangle_{\boldsymbol{M}} \\
& =\left\langle G_{\boldsymbol{M}}\left(F_{1}\right), F_{2}\right\rangle_{\boldsymbol{M}}=\tau_{\boldsymbol{M}}\left(\left[F_{1}\right],\left[F_{2}\right]\right),
\end{aligned}
$$

for all $\left[F_{1}\right],\left[F_{2}\right] \in \mathscr{O}_{\mathrm{c}}(\boldsymbol{M}) / P_{\boldsymbol{M}}\left(\mathscr{O}_{\mathrm{c}}(\boldsymbol{M})\right)$. In the second equality we have used Lemma 5.1 (ii) and in the third equality we have used Lemma 5.6. The functoriality of $\mathfrak{L}$ is induced by the functoriality of $\mathscr{O}_{\mathrm{c}}: \mathrm{SLoc} \rightarrow \mathrm{SVec}$ which has been established in Corollary 5.2 .

We finish this subsection by proving some properties of the functor $\mathfrak{L}:$ SLoc $\rightarrow$ X, which are the axioms of locally covariant quantum field theory [BFV03] applied to classical theories.

Theorem 5.9. For any super-field theory according to Definition 4.2 the associated functor $\mathfrak{L}: \mathrm{SLoc} \rightarrow \mathrm{X}$ satisfies the following properties:

- Locality: For any SLoc-morphism $\chi: \boldsymbol{M} \rightarrow \boldsymbol{M}^{\prime}$, the X-morphism $\mathfrak{L}(\chi): \mathfrak{L}(\boldsymbol{M}) \rightarrow \mathfrak{L}\left(\boldsymbol{M}^{\prime}\right)$ is monic.

- Super-causality: Given two SLoc-morphisms $\boldsymbol{M}_{1} \stackrel{\chi_{1}}{\longrightarrow} \boldsymbol{M} \stackrel{\chi_{2}}{\longleftarrow} \boldsymbol{M}_{2}$ such that the images of the reduced otLor-morphisms $\widetilde{\boldsymbol{M}_{1}} \stackrel{\widetilde{\chi_{1}}}{\longrightarrow} \widetilde{\boldsymbol{M}} \stackrel{\widetilde{\chi_{2}}}{\longleftarrow} \widetilde{\boldsymbol{M}_{2}}$ are causally disjoint, then

$$
\tau_{M}\left(\mathfrak{L}\left(\chi_{1}\right)\left(\mathfrak{L}\left(\boldsymbol{M}_{1}\right)\right), \mathfrak{L}\left(\chi_{2}\right)\left(\mathfrak{L}\left(\boldsymbol{M}_{2}\right)\right)\right)=\{0\} .
$$

- Time-slice axiom: Given any Cauchy SLoc-morphism $3^{3} \chi: \boldsymbol{M} \rightarrow \boldsymbol{M}^{\prime}$, then $\mathfrak{L}(\chi)$ : $\mathfrak{L}(\boldsymbol{M}) \rightarrow \mathfrak{L}\left(\boldsymbol{M}^{\prime}\right)$ is an isomorphism.

\footnotetext{
${ }^{3}$ A Cauchy SLoc-morphism is a SLoc-morphism $\chi: M \rightarrow M^{\prime}$ such that its reduced otLor-morphism $\tilde{\chi}$ : $\widetilde{\boldsymbol{M}} \rightarrow \widetilde{\boldsymbol{M}^{\prime}}$ is Cauchy, i.e. the image of $\widetilde{\chi}$ contains a Cauchy surface in $\widetilde{\boldsymbol{M}^{\prime}}$.
} 
Proof. The locality property is as usual a consequence of $\tau_{M}$ being weakly non-degenerate; indeed, assuming that $\mathfrak{L}(\chi)([F])=0$, for some $[F] \in \mathfrak{L}(\boldsymbol{M})$, we find that

$$
\tau_{M^{\prime}}(\mathfrak{L}(\chi)([H]), \mathfrak{L}(\chi)([F]))=\tau_{M}([H],[F])=0,
$$

for all $[H] \in \mathfrak{L}(\boldsymbol{M})$, and hence $[F]=0$. The super-causality property is a consequence of the support properties of the super-Green's operators, cf. Definition 4.1 (iii).

To show the time-slice axiom, it remains to prove that $\mathfrak{L}(\chi)$ is surjective for any Cauchy SLoc-morphism, which is equivalent to proving that any class $[F] \in \mathfrak{L}\left(\boldsymbol{M}^{\prime}\right)$ has a representative $F^{\prime} \in \mathscr{O}_{\mathrm{c}}\left(\boldsymbol{M}^{\prime}\right)$ with support contained in $\widetilde{\chi}(\widetilde{\boldsymbol{M}}) \subseteq \widetilde{\boldsymbol{M}^{\prime}}$. This follows from a standard argument which we shall now generalize to the case of supermanifolds. Let us take any two non-intersecting Cauchy surfaces $\Sigma^{ \pm} \subset \widetilde{\chi}(\widetilde{\boldsymbol{M}}) \subseteq \widetilde{\boldsymbol{M}^{\prime}}$ of $\widetilde{\boldsymbol{M}^{\prime}}$, such that $\Sigma^{+}$lies in the chronological future of $\Sigma^{-}$. Take the open cover $U^{ \pm}:=I_{M^{\prime}}^{ \pm}\left(\Sigma^{\mp}\right)$ of $\widetilde{\boldsymbol{M}^{\prime}}$ and choose some partition of unity $\rho^{ \pm} \in \mathscr{O}\left(\boldsymbol{M}^{\prime}\right)$ subordinated to this cover (see [CCF11, Proposition 4.2.7] for a proof of existence of partitions of unity on supermanifolds). We choose any representative $F \in \mathscr{O}_{\mathrm{c}}\left(\boldsymbol{M}^{\prime}\right)$ of the class $[F] \in \mathfrak{L}\left(\boldsymbol{M}^{\prime}\right)$ and define

$$
H:=\rho^{-} G_{\boldsymbol{M}^{\prime}}^{+}(F)+\rho^{+} G_{\boldsymbol{M}^{\prime}}^{-}(F) \in \mathscr{O}_{\mathrm{c}}\left(\boldsymbol{M}^{\prime}\right) .
$$

Then $F^{\prime}:=F-P_{\boldsymbol{M}^{\prime}}(H) \in \mathscr{O}_{\mathrm{C}}\left(\boldsymbol{M}^{\prime}\right)$ is a representative of the class $[F]$ with support contained in $\widetilde{\chi}(\widetilde{\boldsymbol{M}}) \subseteq \widetilde{\boldsymbol{M}^{\prime}}$.

\subsection{The quantization functor $\mathfrak{Q}: \mathrm{X} \rightarrow \mathrm{S}^{*} \mathrm{Alg}$}

The quantization is performed by assigning to objects $\boldsymbol{V}$ in X SCCR superalgebras in the case of $\operatorname{dim}(S)$ even and SCAR superalgebras in the case of $\operatorname{dim}(S)$ odd. This reflects the fact that the objects in $\mathrm{X}$ are super-symplectic spaces if $\operatorname{dim}(S)$ is even and super-inner product spaces if $\operatorname{dim}(S)$ is odd. We can perform this construction in one step by using suitable sign and imaginary unit $\mathrm{i} \in \mathbb{C}$ factors (depending on $\operatorname{dim}(S) \bmod 2$ ) in the definitions below.

Let $\boldsymbol{V}=(V, \tau)$ be any object in $\mathrm{X}$. We consider the complexified tensor superalgebra

$$
\mathcal{T}_{\mathbb{C}}(\boldsymbol{V}):=\bigoplus_{n \geq 0} \mathcal{T}_{\mathbb{C}}^{n}(\boldsymbol{V}):=\bigoplus_{n \geq 0} V^{\otimes n} \otimes \mathbb{C}
$$

and denote its product simply by juxtaposition. Notice that $\mathcal{T}_{\mathbb{C}}(\boldsymbol{V})$ is generated (over $\mathbb{C}$ ) by the unit $\mathbb{1}:=1 \in \mathcal{T}_{\mathbb{C}}^{0}(\boldsymbol{V}) \simeq \mathbb{C}$ and the elements $v \cong v \otimes 1 \in \mathcal{T}_{\mathbb{C}}^{1}(\boldsymbol{V})=V \otimes \mathbb{C}$, for all $v \in V$. We equip $\mathcal{T}_{\mathbb{C}}(\boldsymbol{V})$ with the superinvolution which is defined on the generators by $\mathbb{1}^{*}=\mathbb{1}$ and $v^{*}=v$, for all $v \in V$, and extended to all of $\mathcal{T}_{\mathbb{C}}(\boldsymbol{V})$ by $\mathbb{C}$-antilinearity and

$$
\left(a_{1} a_{2}\right)^{*}=(-1)^{\left|a_{1}\right|\left|a_{2}\right|} a_{2}^{*} a_{1}^{*},
$$

for all $\mathbb{Z}_{2}$-parity homogeneous $a_{1}, a_{2} \in \mathcal{T}_{\mathbb{C}}(\boldsymbol{V})$. Using the SVec-morphism $\tau: V \otimes V \rightarrow \mathbb{R}$, we define $\mathcal{I}(\boldsymbol{V})$ to be the two-sided super-*-ideal in $\mathcal{T}_{\mathbb{C}}(\boldsymbol{V})$ that is generated by the elements

$$
v_{1} v_{2}+(-1)^{\operatorname{dim}(S)+1}(-1)^{\left|v_{1}\right|\left|v_{2}\right|} v_{2} v_{1}-\beta \tau\left(v_{1}, v_{2}\right) \mathbb{1},
$$

for all homogeneous generators $v_{1}, v_{2} \in V$, where we have used

$$
\beta:= \begin{cases}\mathrm{i} & , \text { for } \operatorname{dim}(S) \text { even }, \\ 1 & , \text { for } \operatorname{dim}(S) \text { odd } .\end{cases}
$$

Notice that (5.32) describes SCCR if $\operatorname{dim}(S)$ is even and SCAR if $\operatorname{dim}(S)$ is odd. We define the object $\mathfrak{Q}(\boldsymbol{V})$ in $\mathrm{S}^{*} \mathrm{Alg}$ by the quotient

$$
\mathfrak{Q}(\boldsymbol{V}):=\frac{\mathcal{T}_{\mathbb{C}}(\boldsymbol{V})}{\mathcal{I}(\boldsymbol{V})} .
$$


Proposition 5.10. The following assignment is a functor $\mathfrak{Q}: \mathrm{X} \rightarrow \mathrm{S}^{*} \mathrm{Alg}$ : To any object $\boldsymbol{V}$ in $\mathbf{X}$ we assign the object $\mathfrak{Q}(\boldsymbol{V})$ in $\mathrm{S}^{*} \mathrm{Alg}$ given by 5.34) and to any $\mathbf{X}$-morphism $L: \boldsymbol{V} \rightarrow \boldsymbol{V}^{\prime}$ we assign the $\mathrm{S}^{*} \mathrm{Alg}$-morphism $\mathfrak{Q}(L): \mathfrak{Q}(\boldsymbol{V}) \rightarrow \mathfrak{Q}\left(\boldsymbol{V}^{\prime}\right)$ that is specified by defining on the generators $\mathfrak{Q}(L)(v):=L(v)$, for all $v \in V$.

Proof. By our constructions above, we already know that $\mathfrak{Q}(\boldsymbol{V})$ is an object in $\mathrm{S}^{*} \mathrm{Alg}$, for all objects $\boldsymbol{V}$ in X. It remains to show that $\mathfrak{Q}(L): \mathfrak{Q}(\boldsymbol{V}) \rightarrow \mathfrak{Q}\left(\boldsymbol{V}^{\prime}\right)$ specified above is well-defined, i.e. that it preserves the two-sided super-*-ideals. This is a consequence of the explicit form of the generators of these ideals (5.32) and the fact that $L: \boldsymbol{V} \rightarrow \boldsymbol{V}^{\prime}$ satisfies $\tau^{\prime} \circ(L \otimes L)=\tau$.

\subsection{The locally covariant quantum field theory $\mathfrak{A}:$ SLoc $\rightarrow \mathrm{S}^{*} \mathrm{Alg}$}

We compose the functors $\mathfrak{L}: \mathrm{SLoc} \rightarrow \mathrm{X}$ and $\mathfrak{Q}: \mathrm{X} \rightarrow \mathrm{S}^{*}$ Alg in order to define the functor

$$
\mathfrak{A}:=\mathfrak{Q} \circ \mathfrak{L}: \mathrm{SLoc} \longrightarrow \mathrm{S}^{*} \text { Alg } .
$$

This functor satisfies a supergeometric modification of the axioms of locally covariant quantum field theory [BFV03].

Theorem 5.11. For any super-field theory according to Definition 4.2 the associated functor $\mathfrak{A}:$ SLoc $\rightarrow \mathrm{S}^{*}$ Alg satisfies the following properties:

- Locality: For any SLoc-morphism $\chi: \boldsymbol{M} \rightarrow \boldsymbol{M}^{\prime}$, the $\mathrm{S}^{*}$ Alg-morphism $\mathfrak{A}(\chi): \mathfrak{A}(\boldsymbol{M}) \rightarrow$ $\mathfrak{A}\left(\boldsymbol{M}^{\prime}\right)$ is monic.

- Super-causality: Given two SLoc-morphisms $\boldsymbol{M}_{1} \stackrel{\chi_{1}}{\longrightarrow} \boldsymbol{M} \stackrel{\chi_{2}}{\longleftarrow} \boldsymbol{M}_{2}$ such that the images

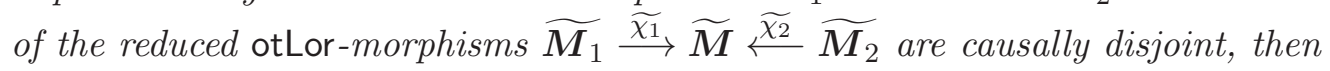

$$
a_{1} a_{2}+(-1)^{\operatorname{dim}(S)+1}(-1)^{\left|a_{1}\right|\left|a_{2}\right|} a_{2} a_{1}=0,
$$

for all homogeneous $a_{1} \in \mathfrak{A}\left(\chi_{1}\right)\left(\mathfrak{A}\left(\boldsymbol{M}_{1}\right)\right)$ and $a_{2} \in \mathfrak{A}\left(\chi_{2}\right)\left(\mathfrak{A}\left(\boldsymbol{M}_{2}\right)\right)$.

- Time-slice axiom: Given any Cauchy SLoc-morphism $\chi: \boldsymbol{M} \rightarrow \boldsymbol{M}^{\prime}$, then $\mathfrak{A}(\chi)$ : $\mathfrak{A}(\boldsymbol{M}) \rightarrow \mathfrak{A}\left(\boldsymbol{M}^{\prime}\right)$ is a $\mathrm{S}^{*}$ Alg-isomorphism.

Proof. All properties listed above follow by standard arguments from the corresponding properties of the classical theory given in Theorem [5.9. Let us briefly give a sketch or reference: The locality property follows by using the techniques summarized in [FV12, Appendix A]. The super-causality property for $a_{1}$ and $a_{2}$ being two generators follows explicitly from the form of the two-sided super-*-ideal (5.32) and for generic $a_{1}$ and $a_{2}$ by expressing these elements in terms of generators and using iteratively the super-causality property for the generators. The time-slice axiom for $\mathfrak{A}=\mathfrak{Q} \circ \mathfrak{L}$ follows since functors (here $\mathfrak{Q}$ ) preserve isomorphisms.

Remark 5.12. Notice that the super-causality property (5.36) is similar to the graded-causality property encountered in fermionic quantum field theories, see e.g. [BG11. In particular, if $\operatorname{dim}(S)$ is even then (5.36) implies that two even elements commute and two odd elements anti-commute whenever they are spacelike separated. In an ordinary (i.e. non-supergeometric) quantum field theory one usually postulates that only the even elements of the algebras are true physical observables, which includes in particular bilinear terms in fermionic quantum fields such as the stress-energy tensor. A similar construction is also possible in our present description of super-QFTs: We may define a new functor $\mathfrak{A}_{0}:$ SLoc $\rightarrow{ }^{*} \mathrm{Alg}$ (the even part of the super-QFT $\mathfrak{A}: \mathrm{SLoc} \rightarrow \mathrm{S}^{*} \mathrm{Alg}$ ) to the category of ordinary $*$-algebras by assigning to any object $\boldsymbol{M}$ in SLoc the even sub-*-algebra $\mathfrak{A}_{0}(\boldsymbol{M})$ of $\mathfrak{A}(\boldsymbol{M})$ and to any SLoc-morphism $\chi: \boldsymbol{M} \rightarrow \boldsymbol{M}^{\prime}$ the restriction of $\mathfrak{A}(\chi): \mathfrak{A}(\boldsymbol{M}) \rightarrow \mathfrak{A}\left(\boldsymbol{M}^{\prime}\right)$ to $\mathfrak{A}_{0}(\boldsymbol{M})$. (Because $\mathfrak{A}(\chi)$ preserves $\mathbb{Z}_{2}$-parity we have that $\mathfrak{A}_{0}(\chi):=\left.\mathfrak{A}(\chi)\right|_{\mathfrak{A}_{0}(\boldsymbol{M})}: \mathfrak{A}_{0}(\boldsymbol{M}) \rightarrow \mathfrak{A}_{0}\left(\boldsymbol{M}^{\prime}\right)$ maps to $\mathfrak{A}_{0}\left(\boldsymbol{M}^{\prime}\right)$.) The functor $\mathfrak{A}_{0}:$ SLoc $\rightarrow{ }^{*}$ Alg then satisfies the ordinary locality, causality and time-slice axiom of locally covariant quantum field theory [BFV03]. 
We conclude this section by constructing a locally covariant quantum field for the functor $\mathfrak{A}: \operatorname{SLoc} \rightarrow \mathrm{S}^{*}$ Alg, which establishes a connection to the data specifying a super-field theory in Definition 4.2, Let us consider the functor $\mathscr{O}_{\mathrm{c}}: \mathrm{SLoc} \rightarrow \mathrm{SVec}$ and regard $\mathfrak{A}$ also as a functor to SVec (denoted by the same symbol) by composing with the forgetful functor. There is a natural transformation $\Phi: \mathscr{O}_{\mathrm{c}} \Rightarrow \mathfrak{A}$ of functors from SLoc to SVec with components given by the SVec-morphisms

$$
\Phi_{M}: \mathscr{O}_{\mathrm{c}}(\boldsymbol{M}) \longrightarrow \mathfrak{A}(\boldsymbol{M}), \quad F \longmapsto[F]
$$

In analogy to BFV03 we shall interpret $\Phi_{\boldsymbol{M}}(F) \in \mathfrak{A}(\boldsymbol{M})$, for $F \in \mathscr{O}_{\mathrm{c}}(\boldsymbol{M})$, as a smeared linear hermitian superfield operator on $\boldsymbol{M}$. The connection to the data in Definition 4.2 is established by noticing that the superfield operators satisfy the equations of motion (in weak form)

$$
\Phi_{M}\left(P_{M}(F)\right)=0,
$$

for all $F \in \mathscr{O}_{\mathrm{c}}(\boldsymbol{M})$ and all objects $\boldsymbol{M}$ in SLoc. Moreover, they satisfy the super-canonical (anti)commutation relations

$$
\Phi_{\boldsymbol{M}}\left(F_{1}\right) \Phi_{\boldsymbol{M}}\left(F_{2}\right)+(-1)^{\operatorname{dim}(S)+1}(-1)^{\left|F_{1}\right|\left|F_{2}\right|} \Phi_{\boldsymbol{M}}\left(F_{2}\right) \Phi_{\boldsymbol{M}}\left(F_{1}\right)=\beta \tau_{\boldsymbol{M}}\left(F_{1}, F_{2}\right),
$$

for all homogeneous $F_{1}, F_{2} \in \mathscr{O}_{\mathrm{c}}(\boldsymbol{M})$ and all objects $\boldsymbol{M}$ in SLoc. We recall that $\beta=\mathrm{i}$ if $\operatorname{dim}(S)$ is even and $\beta=1$ if $\operatorname{dim}(S)$ is odd.

Remark 5.13. For any object $\boldsymbol{M}$ in SLoc we have a decomposition $\mathscr{O}_{\mathrm{c}}(\boldsymbol{M})=\mathscr{O}_{\mathrm{c}}(\boldsymbol{M})_{0} \oplus$ $\mathscr{O}_{\mathrm{c}}(\boldsymbol{M})_{1}$ into the even and odd part. We can define new super-vector spaces $\mathscr{O}_{\mathrm{c}}^{\text {even }}(\boldsymbol{M}):=$ $\mathscr{O}_{\mathrm{c}}(\boldsymbol{M})_{0} \oplus 0$ and $\mathscr{O}_{\mathrm{c}}^{\text {odd }}(\boldsymbol{M}):=0 \oplus \mathscr{O}_{\mathrm{c}}(\boldsymbol{M})_{1}$ and notice that $\mathscr{O}_{\mathrm{c}}^{\text {even }}:$ SLoc $\rightarrow$ SVec and $\mathscr{O}_{\mathrm{c}}^{\text {odd }}:$ $\mathrm{SLoc} \rightarrow$ SVec are subfunctors of $\mathscr{O}_{\mathrm{c}}: \mathrm{SLoc} \rightarrow$ SVec. (The latter statement is due to the fact that the push-forwards $\mathscr{O}_{\mathrm{c}}(\chi)=\chi_{*}$ preserve the $\mathbb{Z}_{2}$-parity.) Consequently, our locally covariant quantum field $\Phi: \mathscr{O}_{\mathrm{c}} \Rightarrow \mathfrak{A}$ decomposes into two natural transformation $\Phi^{\text {even }}: \mathscr{O}_{\mathrm{c}}^{\text {even }} \Rightarrow \mathfrak{A}$ and $\Phi^{\text {odd }}: \mathscr{O}_{\mathrm{c}}^{\text {odd }} \Rightarrow \mathfrak{A}$, which describe within our physical interpretation the even and odd component quantum fields of the superfield $\Phi$. The appearance of the even and odd quantum fields is an undesirable feature, which indicates that our formulation does not appropriately capture supersymmetry transformations. In fact, supersymmetry transformations are supposed to mix the even and odd component fields, hence allowing neither of them to be a natural transformation, i.e. a locally covariant quantum field. It is the goal of the next section to 'enrich' (in a mathematically precise way) the categories and functors appearing in our construction in order to capture also supersymmetry transformations.

\section{Axiomatic definition of enriched super-field theories}

Motivated by the shortcomings of our present theory, which have been summarized in Remark 5.13, we shall now systematically 'enrich' all categories, functors and natural transformations appearing in the Definition 4.2 of super-field theories. A suitable mathematical framework is that of enriched category theory, see e.g. [Kel82, BS00] and also Appendix \$ for a brief introduction to the basic concepts. Loosely speaking, in an ordinary category the morphisms between two objects have to form a set and in an enriched category the morphisms between two objects are allowed to be an object in another (monoidal) category. Enriched functors and natural transformations are then defined by a suitable generalization of the standard concepts of functors and natural transformations in ordinary category theory. In our definition of enriched super-field theories, as well as in the construction of the corresponding enriched super-QFTs in Section 7, we shall consider enriched categories over the monoidal category SSet of supersets, which we also call SSet-categories. Again loosely speaking, while an ordinary set is determined by its points, a superset is determined by its superpoints. To make precise the 
notion of supersets, we shall use the category theoretical approach to supergeometry proposed by Schwarz [Shv84] and Molotkov [Mol84], and developed in detail by Sachse [Sac08], see also SW11, AL12].

\subsection{The monoidal category SSet of supersets}

For better understanding the concept of supersets, it will be helpful to view ordinary sets from a categorical perspective. Let $A$ be any set. Then $A$ is determined by its points, which can be described by maps $x: \mathrm{pt} \rightarrow A$ from a (once and for all fixed) singleton set $\mathrm{pt}:=\{\star\}$ to the set $A$. In other words, the points of $A$ are described by the morphism set $\operatorname{Hom}_{\text {Set }}(\mathrm{pt}, A)$. Using the usual composition of maps, any map between two sets $f: A \rightarrow B$ induces a map between the morphism sets

$$
\operatorname{Hom}_{\text {Set }}(\mathrm{pt}, A) \longrightarrow \operatorname{Hom}_{\text {Set }}(\mathrm{pt}, B), x \longmapsto f \circ x .
$$

Let $\mathrm{Pt}$ be the category consisting of the single object $\mathrm{pt}$ and the single morphism $\mathrm{id}_{\mathrm{pt}}$. Then the morphism set above can be regarded as a functor $\operatorname{Hom}_{\text {Set }}(\cdot, A): \mathrm{Pt}^{\mathrm{op}} \rightarrow$ Set (in foresight we use here the opposite category $\left.\mathrm{Pt}^{\mathrm{op}}\right)$ and the map (6.1) as a natural transformation $\operatorname{Hom}_{\mathrm{Set}}(\cdot, A) \Rightarrow$ $\operatorname{Hom}_{\text {Set }}(\cdot, B)$ between functors from $\mathrm{Pt}^{\mathrm{op}}$ to Set. What this means is that we have constructed a functor

$$
\text { Set } \longrightarrow \operatorname{Fun}\left(\mathrm{Pt}^{\mathrm{op}}, \mathrm{Set}\right)
$$

from the category of sets to the category of functors from $\mathrm{Pt}^{\mathrm{op}}$ to Set. Notice that the functor (6.2) is an equivalence between the categories Set and Fun ( $\mathrm{Pt}^{\mathrm{op}}$, Set). In other words, we can choose freely if we want to work with the usual category Set of sets or with the functor category Fun $\left(\mathrm{Pt}^{\mathrm{op}}\right.$, Set $)$.

Motivated by this functorial point of view, we shall define the category of supersets as the functor category Fun $\left(\mathrm{SPt}^{\mathrm{op}}\right.$, Set), where $\mathrm{SPt}$ is the following category of superpoints:

Definition 6.1. The category SPt consists of the following objects and morphisms:

- The objects are given by the supermanifolds $\mathrm{pt}_{n}:=\left(\mathrm{pt}, \Lambda_{n}\right)$, where $\Lambda_{n}:=\bigwedge^{\bullet} \mathbb{R}^{n}$ is the real Grassmann algebra over $\mathbb{R}^{n}$ and $n \in \mathbb{N}^{0}$.

- The morphisms $\lambda: \mathrm{pt}_{n} \rightarrow \mathrm{pt}_{m}$ are all supermanifold morphisms.

The category SSet of supersets is defined as the functor category

$$
\text { SSet }:=\operatorname{Fun}\left(\mathrm{SPt}^{\mathrm{op}}, \text { Set }\right) \text {. }
$$

Remark 6.2. In [Sac08], the category of superpoints is defined as the full subcategory of SMan with objects given by all supermanifolds whose underlying topological space is a singleton. This category is equivalent to our category SPt and moreover we have that $\mathrm{SPt}^{\mathrm{op}}$ is equivalent to the category of finite-dimensional real Grassmann algebras Gr. Hence, our category of supersets (6.3) is equivalent to the functor category Fun(Gr, Set), which is used for example in [Sac08.

Recall that the category Set of ordinary sets is a monoidal category with bifunctor $\times$ : Set $\times$ Set $\rightarrow$ Set given by the Cartesian product and unit object given by the singleton set pt. By a general construction, the monoidal structure on Set induces a monoidal structure on the functor category Fun $\left(\mathrm{SPt}^{\mathrm{op}}\right.$, Set) and hence on the category SSet of supersets. Let us briefly recall this construction and give explicit formulas. We define a bifunctor (denoted with a slight abuse of notation also by $\times$ )

$$
\times: \text { SSet } \times \text { SSet } \longrightarrow \text { SSet }
$$


by assigning to any object $\left(\mathfrak{F}: \mathrm{SPt}^{\mathrm{op}} \rightarrow \mathrm{Set}, \mathfrak{F}^{\prime}: \mathrm{SPt}{ }^{\mathrm{op}} \rightarrow\right.$ Set $)$ in SSet $\times$ SSet the object $\mathfrak{F} \times \mathfrak{F}^{\prime}: \mathrm{SPt}^{\mathrm{op}} \rightarrow$ Set in SSet, which is the functor specified on objects $\mathrm{pt}_{n}$ in $\mathrm{SPt}^{\mathrm{op}}$ by

$$
\left(\mathfrak{F} \times \mathfrak{F}^{\prime}\right)\left(\mathrm{pt}_{n}\right):=\mathfrak{F}\left(\mathrm{pt}_{n}\right) \times \mathfrak{F}^{\prime}\left(\mathrm{pt}_{n}\right)
$$

and on $\mathrm{SPt}^{\mathrm{op}}$-morphisms $\lambda^{\mathrm{op}}: \mathrm{pt}_{n} \rightarrow \mathrm{pt}_{m}$ by

$$
\left(\mathfrak{F} \times \mathfrak{F}^{\prime}\right)\left(\lambda^{\mathrm{op}}\right):=\mathfrak{F}\left(\lambda^{\mathrm{op}}\right) \times \mathfrak{F}^{\prime}\left(\lambda^{\mathrm{op}}\right):\left(\mathfrak{F} \times \mathfrak{F}^{\prime}\right)\left(\mathrm{pt}_{n}\right) \longrightarrow\left(\mathfrak{F} \times \mathfrak{F}^{\prime}\right)\left(\mathrm{pt}_{m}\right) .
$$

To any morphism $\left(\eta: \mathfrak{F} \Rightarrow \mathfrak{G}, \eta^{\prime}: \mathfrak{F}^{\prime} \Rightarrow \mathfrak{G}^{\prime}\right)$ in SSet $\times$ SSet we assign the morphism $\eta \times \eta^{\prime}:$ $\mathfrak{F} \times \mathfrak{F}^{\prime} \Rightarrow \mathfrak{G} \times \mathfrak{G}^{\prime}$ in SSet which is given by the natural transformation with components

$$
\left(\eta \times \eta^{\prime}\right)_{\mathrm{pt}_{n}}:=\eta_{\mathrm{pt}_{n}} \times \eta_{\mathrm{pt}_{n}}^{\prime}:\left(\mathfrak{F} \times \mathfrak{F}^{\prime}\right)\left(\mathrm{pt}_{n}\right) \longrightarrow\left(\mathfrak{G} \times \mathfrak{G}^{\prime}\right)\left(\mathrm{pt}_{n}\right),
$$

for all objects $\mathrm{pt}_{n}$ in $\mathrm{SPt}^{\mathrm{op}}$. The unit object in SSet is the functor $\mathfrak{I}: \mathrm{SPt}^{\mathrm{op}} \rightarrow$ Set specified on objects $\mathrm{pt}_{n}$ and morphisms $\lambda^{\mathrm{op}}: \mathrm{pt}_{n} \rightarrow \mathrm{pt}_{m}$ in $\mathrm{SPt}^{\mathrm{op}}$ by

$$
\Im\left(\mathrm{pt}_{n}\right):=\mathrm{pt}, \quad \Im\left(\lambda^{\mathrm{op}}\right):=\mathrm{id}_{\mathrm{pt}} .
$$

In summary, we have

Proposition 6.3. The category SSet of supersets is a monoidal category with bifunctor $\times$ : SSet $\times$ SSet $\rightarrow$ SSet defined by (6.5) and unit object $\mathfrak{I}$ defined by (6.6).

\subsection{The SSet-category eSLoc}

Let us choose as in Definition 4.2 any full subcategory SLoc of ghSCart. The goal of this subsection is to define a SSet-category eSLoc, such that the objects in eSLoc coincide with those in SLoc and that the morphism supersets in eSLoc enrich (in a suitable way) the ordinary morphism sets in SLoc. The main feature of this enrichment will be that supersymmetry transformations appear as superpoints of the morphism supersets, see Section 8 for explicit examples.

Before we can define the SSet-category eSLoc we need some preparations. A supermanifold $M$ can be described in the framework of supersets (6.3) by the functor $\operatorname{Hom}_{\mathrm{SMan}}(\cdot, M)$ : $\mathrm{SPt}^{\mathrm{op}} \rightarrow$ Set. We will not describe the details of this approach (see Shv84, Mol84, Sac08, Sac09), but we make use of an equivalent picture: $\operatorname{Hom}_{\mathrm{SMan}}\left(\mathrm{pt}_{n}, M\right)$ clearly coincides with the set sections of the trivial super-fibre bundle $\mathrm{pt}_{n} \times M \rightarrow \mathrm{pt}_{n}$ and natural transformations $\operatorname{Hom}_{\mathrm{SMan}}(\cdot, M) \rightarrow \operatorname{Hom}_{\mathrm{SMan}}\left(\cdot, M^{\prime}\right)$ correspond to super-fibre bundle morphisms. We will discuss the basic properties of this "family point of view" and refer to the literature Sac09, Chapter 3.3] and [Del99, §2.8 and §2.9] for more details.

Given any object $M$ in SMan and any object $\mathrm{pt}_{n}$ in $\mathrm{SPt}^{\mathrm{op}}$, we can consider the product supermanifold $\mathrm{pt}_{n} \times M=\left(\widetilde{M}, \Lambda_{n} \otimes \mathscr{O}_{M}\right)$ together with the projection SMan-morphism $\mathrm{pr}_{\mathrm{pt}_{n} \times M, \mathrm{pt}_{n}}: \mathrm{pt}_{n} \times M \rightarrow \mathrm{pt}_{n}$ onto the first factor. The pair $\left(\mathrm{pt}_{n} \times M, \mathrm{pr}_{\mathrm{pt}_{n} \times M, \mathrm{pt}_{n}}\right)$ is typically called $a \mathrm{pt}_{n}$-relative supermanifold and denoted by the compact notation $M / \mathrm{pt}_{n}$. $\mathrm{A} a \mathrm{pt}_{n}$ relative SMan-morphism (in short: SMan/pt ${ }_{n}$-morphism) $\chi: M / \mathrm{pt}_{n} \rightarrow M^{\prime} / \mathrm{pt}_{n}$ between two $\mathrm{pt}_{n}$-relative supermanifolds is a SMan-morphism $\chi: \mathrm{pt}_{n} \times M \rightarrow \mathrm{pt}_{n} \times M^{\prime}$ between the product supermanifolds which preserves the projections, i.e. the diagram

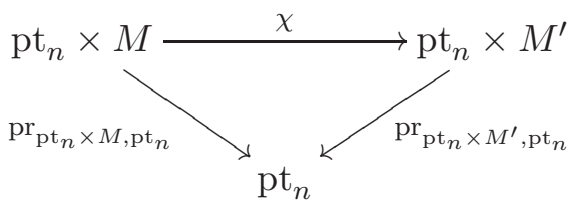


in SMan commutes. Explicitly, a SMan-morphism $\chi: \mathrm{pt}_{n} \times M \rightarrow \mathrm{pt}_{n} \times M^{\prime}$ is a SMan $/ \mathrm{pt}_{n^{-}}$ morphism if and only if $\chi^{*}(\zeta \otimes \mathbb{1})=\zeta \otimes \mathbb{1}$, for all $\zeta \in \Lambda_{n}$. Notice that the identity $\operatorname{id}_{\mathrm{pt}_{n} \times M}$ : $M / \mathrm{pt}_{n} \rightarrow M / \mathrm{pt}_{n}$ is a SMan/pt ${ }_{n}$-morphism and that two SMan/ $/ \mathrm{pt}_{n}$-morphisms $\chi: M / \mathrm{pt}_{n} \rightarrow$ $M^{\prime} / \mathrm{pt}_{n}$ and $\chi^{\prime}: M^{\prime} / \mathrm{pt}_{n} \rightarrow M^{\prime \prime} / \mathrm{pt}_{n}$ can be composed, i.e. $\chi^{\prime} \circ \chi: M / \mathrm{pt}_{n} \rightarrow M^{\prime \prime} / \mathrm{pt}_{n}$ is a $\mathrm{SMan} / \mathrm{pt}_{n}$-morphism. Using the defining property (6.7), the set of all SMan $/ \mathrm{pt}_{n}$-morphisms $\chi: M / \mathrm{pt}_{n} \rightarrow M^{\prime} / \mathrm{pt}_{n}$ can be easily characterized.

Lemma 6.4. Let $M$ and $M^{\prime}$ be any two objects in SMan and $\mathrm{pt}_{n}$ any object in $\mathrm{SPt}^{\mathrm{op}}$. Then the map

$$
\begin{aligned}
\alpha_{\mathrm{pt}_{n}}: \operatorname{Hom}_{\mathrm{SMan} \mathrm{pt}_{n}}\left(M / \mathrm{pt}_{n}, M^{\prime} / \mathrm{pt}_{n}\right) & \longrightarrow \operatorname{Hom}_{\mathrm{SMan}}\left(\mathrm{pt}_{n} \times M, M^{\prime}\right), \\
\left(\chi: \mathrm{pt}_{n} \times M \rightarrow \mathrm{pt}_{n} \times M^{\prime}\right) & \longmapsto\left(\mathrm{pr}_{\mathrm{pt}_{n} \times M^{\prime}, M^{\prime}} \circ \chi: \mathrm{pt}_{n} \times M \rightarrow M^{\prime}\right),
\end{aligned}
$$

is a bijection of sets, where $\mathrm{pr}_{\mathrm{pt}_{n} \times M^{\prime}, M^{\prime}}: \mathrm{pt}_{n} \times M^{\prime} \rightarrow M^{\prime}$ denotes the projection SMan-morphism on the second factor. In fact, its inverse is given by

$$
\begin{aligned}
\alpha_{\mathrm{pt}_{n}}^{-1}: \operatorname{Hom}_{\mathrm{SMan}}\left(\mathrm{pt}_{n} \times M, M^{\prime}\right) & \longrightarrow \operatorname{Hom}_{\mathrm{SMan} \mathrm{pt}_{n}}\left(M / \mathrm{pt}_{n}, M^{\prime} / \mathrm{pt}_{n}\right), \\
\left(\psi: \mathrm{pt}_{n} \times M \rightarrow M^{\prime}\right) & \longmapsto\left(\left(\mathrm{id}_{\mathrm{pt}_{n}}, \psi\right): \mathrm{pt}_{n} \times M \rightarrow \mathrm{pt}_{n} \times M^{\prime}\right) .
\end{aligned}
$$

We next show that the assignment $\mathrm{pt}_{n} \mapsto \operatorname{Hom}_{\mathrm{SMan} / \mathrm{pt}_{n}}\left(M / \mathrm{pt}_{n}, M^{\prime} / \mathrm{pt}_{n}\right)$ defines a functor $\mathrm{SPt}^{\mathrm{op}} \rightarrow$ Set, which is basically the functor used in [Sac09, Han14] to define super-mapping spaces. Given any two objects $M$ and $M^{\prime}$ in SMan and any $\mathrm{SPt}^{\mathrm{op}}$-morphism $\lambda^{\mathrm{op}}: \mathrm{pt}_{n} \rightarrow \mathrm{pt}_{m}$ (i.e. a SPt-morphism $\lambda: \mathrm{pt}_{m} \rightarrow \mathrm{pt}_{n}$ ) we can define a map of sets

$$
\begin{aligned}
\operatorname{Hom}_{S M a n}\left(\mathrm{pt}_{n} \times M, M^{\prime}\right) & \longrightarrow \operatorname{Hom}_{S M a n}\left(\mathrm{pt}_{m} \times M, M^{\prime}\right), \\
\left(\psi: \mathrm{pt}_{n} \times M \rightarrow M^{\prime}\right) & \longmapsto\left(\psi \circ\left(\lambda \times \mathrm{id}_{M}\right): \mathrm{pt}_{m} \times M \rightarrow M^{\prime}\right) .
\end{aligned}
$$

Using also Lemma 6.4 we obtain a map of sets

$$
\begin{aligned}
\lambda_{*}^{\mathrm{op}}: \operatorname{Hom}_{\mathrm{SMan}_{\mathrm{pt}}}\left(M / \mathrm{pt}_{n}, M^{\prime} / \mathrm{pt}_{n}\right) & \longrightarrow \operatorname{Hom}_{\mathrm{SMan}_{\mathrm{pt}}}\left(M / \mathrm{pt}_{m}, M^{\prime} / \mathrm{pt}_{m}\right), \\
\chi & \longmapsto \alpha_{\mathrm{pt}_{m}}^{-1}\left(\alpha_{\mathrm{pt}_{n}}(\chi) \circ\left(\lambda \times \mathrm{id}_{M}\right)\right),
\end{aligned}
$$

which describes how relative SMan-morphisms behave under the exchange of superpoints. The following properties can be easily derived from (6.11). We therefore can omit the proof.

Lemma 6.5. (i) For any identity $\mathrm{SPt}^{\mathrm{op}}$-morphism $\lambda^{\mathrm{op}}=\mathrm{id}_{\mathrm{pt}_{n}}: \mathrm{pt}_{n} \rightarrow \mathrm{pt}_{n}$ the map $\lambda_{*}^{\mathrm{op}}$ is the identity. For any two $\mathrm{SPt}^{\mathrm{op}}-$ morphisms $\lambda^{\mathrm{op}}: \mathrm{pt}_{n} \rightarrow \mathrm{pt}_{m}$ and $\lambda^{\prime \mathrm{op}}: \mathrm{pt}_{m} \rightarrow \mathrm{pt}_{l}$ we have that $\left(\lambda^{\prime \mathrm{op}} \circ \mathrm{op} \lambda^{\mathrm{op}}\right)_{*}=\lambda_{*}^{\mathrm{op}} \circ \lambda_{*}^{\mathrm{op}}$.

(ii) $\lambda_{*}^{\mathrm{op}}$ preserves identities and compositions, i.e.

$$
\lambda_{*}^{\mathrm{op}}\left(\mathrm{id}_{\mathrm{pt}_{n} \times M}\right)=\mathrm{id}_{\mathrm{pt}_{m} \times M}, \quad \lambda_{*}^{\mathrm{op}}\left(\chi^{\prime} \circ \chi\right)=\lambda_{*}^{\mathrm{op}}\left(\chi^{\prime}\right) \circ \lambda_{*}^{\mathrm{op}}(\chi),
$$

for all objects $M$ in SMan and all SMan/pt ${ }_{n}$-morphisms $\chi: M / \mathrm{pt}_{n} \rightarrow M^{\prime} / \mathrm{pt}_{n}$ and $\chi^{\prime}: M^{\prime} / \mathrm{pt}_{n} \rightarrow M^{\prime \prime} / \mathrm{pt}_{n}$.

(iii) $\lambda_{*}^{\mathrm{op}}$ preserves isomorphisms, i.e. $\chi: M / \mathrm{pt}_{n} \rightarrow M^{\prime} / \mathrm{pt}_{n}$ is a $\mathrm{SMan} / \mathrm{pt}_{n}$-isomorphism if and only if $\lambda_{*}^{\mathrm{op}}(\chi): M / \mathrm{pt}_{m} \rightarrow M^{\prime} / \mathrm{pt}_{m}$ is a SMan/pt ${ }_{m}$-isomorphism.

We shall also require a relative notion of differential geometry on $\mathrm{pt}_{n}$-relative supermanifolds $M / \mathrm{pt}_{n}$. Let $\operatorname{Der}_{\mathrm{pt}_{n} \times M}$ be the superderivation sheaf of the product supermanifold $\mathrm{pt}_{n} \times M$ and $U \subseteq \widetilde{M}$ be any open subset. A superderivation $X \in \operatorname{Der}_{\mathrm{pt}_{n} \times M}(U)$ is called a $\mathrm{pt}_{n}$-relative superderivation provided that $X(\zeta \otimes \mathbb{1})=0$, for all $\zeta \in \Lambda_{n}$. The $\mathrm{pt}_{n}$-relative superderivations form a subsheaf $\operatorname{Der}_{M / \mathrm{pt}_{n}}$ of left $\mathscr{O}_{\mathrm{pt}_{n} \times M}$-supermodules of $\operatorname{Der}_{\mathrm{pt}_{n} \times M}$, which is isomorphic 
to $\Lambda_{n} \otimes \operatorname{Der}_{M}$. The dual $\Omega_{M / \mathrm{pt}_{n}}^{1}:=\underline{\operatorname{Hom}}_{\mathfrak{P t}_{n} \times M}\left(\operatorname{Der}_{M / \mathrm{pt}_{n}}, \mathscr{O}_{\mathrm{pt}_{n} \times M}\right)$ of the $\mathrm{pt}_{n}$-relative superderivation sheaf $\operatorname{Der}_{M / \mathrm{pt}_{n}}$ is called the $\mathrm{pt}_{n}$-relative super-one-form sheaf and it is isomorphic to $\Lambda_{n} \otimes \Omega_{M}^{1}$. The $\mathrm{pt}_{n}$-relative differential $\mathrm{d}_{M / \mathrm{pt}_{n}}: \mathscr{O}_{\mathrm{pt}_{n} \times M} \rightarrow \Omega_{M / \mathrm{pt}_{n}}^{1}$ is defined as in the nonrelative case and it can be identified with $\operatorname{id}_{\Lambda_{n}} \otimes \mathrm{d}: \Lambda_{n} \otimes \mathscr{O}_{M} \rightarrow \Lambda_{n} \otimes \Omega_{M}^{1}$, where d $: \mathscr{O}_{M} \rightarrow \Omega_{M}^{1}$ is the usual differential. Loosely speaking, we obtain the $\mathrm{pt}_{n}$-relative geometric objects on $M / \mathrm{pt}_{n}$ by $\Lambda_{n}$-superlinear extension of the ones on $M$. As a consequence, given any object $\boldsymbol{M}=(M, \Omega, E)$ in SLoc and any object $\mathrm{pt}_{n}$ in $\mathrm{SPt}^{\mathrm{op}}$, we can assign a $\mathrm{pt}_{n}$-relative supermanifold $M / \mathrm{pt}_{n}$ together with $\mathrm{pt}_{n}$-relative super-one-forms $\mathbb{1} \otimes \Omega \in \Lambda_{n} \otimes \Omega^{1}(M, \mathfrak{s p i n}) \simeq \Omega^{1}\left(M / \mathrm{pt}_{n}, \mathfrak{s p i n}\right)$ and $\mathbb{1} \otimes E \in \Lambda_{n} \otimes \Omega^{1}(M, \mathfrak{t}) \simeq \Omega^{1}\left(M / \mathrm{pt}_{n}, \mathfrak{t}\right)$.

With these preparations we can now define the SSet-category eSLoc.

Definition 6.6. The SSet-category eSLoc is given by the following data:

- The objects are all objects $M=(M, \Omega, E)$ in SLoc.

- For any two objects $\boldsymbol{M}$ and $\boldsymbol{M}^{\prime}$ in eSLoc, the object of morphisms from $\boldsymbol{M}$ to $\boldsymbol{M}^{\prime}$ is given by the following functor eSLoc $\left(\boldsymbol{M}, \boldsymbol{M}^{\prime}\right): \mathrm{SPt}^{\mathrm{op}} \rightarrow$ Set: For any object $\mathrm{pt}_{n}$ in $\mathrm{SPt}^{\mathrm{op}}$ we define $\operatorname{eSLoc}\left(\boldsymbol{M}, \boldsymbol{M}^{\prime}\right)\left(\mathrm{pt}_{n}\right)$ to be the set of all SMan/pt ${ }_{n}$-morphisms $\chi: M / \mathrm{pt}_{n} \rightarrow$ $M^{\prime} / \mathrm{pt}_{n}$, such that

1. $\widetilde{\chi}: \widetilde{M} \rightarrow \widetilde{M^{\prime}}$ is an open embedding with causally compatible image in the reduced oriented and time-oriented Lorentz manifold $\widetilde{\boldsymbol{M}^{\prime}}$,

2. $\chi: M /\left.\mathrm{pt}_{n} \rightarrow M^{\prime}\right|_{\widetilde{\chi}(\widetilde{M})} / \mathrm{pt}_{n}$ is a SMan/pt ${ }_{n}$-isomorphism,

3. the $\mathrm{pt}_{n}$-relative super-Cartan structures are preserved, i.e. $\chi^{*}\left(\mathbb{1} \otimes \Omega^{\prime}\right)=\mathbb{1} \otimes \Omega$ and $\chi^{*}\left(\mathbb{1} \otimes E^{\prime}\right)=1 \otimes E$.

For any $\mathrm{SPt}^{\mathrm{op}}$ - morphism $\lambda^{\mathrm{op}}: \mathrm{pt}_{n} \rightarrow \mathrm{pt}_{m}$ we define the map of sets

$$
\operatorname{eSLoc}\left(\boldsymbol{M}, \boldsymbol{M}^{\prime}\right)\left(\lambda^{\mathrm{op}}\right):=\lambda_{*}^{\mathrm{op}}: \operatorname{eSLoc}\left(\boldsymbol{M}, \boldsymbol{M}^{\prime}\right)\left(\mathrm{pt}_{n}\right) \longrightarrow \operatorname{eSLoc}\left(\boldsymbol{M}, \boldsymbol{M}^{\prime}\right)\left(\mathrm{pt}_{m}\right)
$$

where $\lambda_{*}^{\text {op }}$ is given in (6.11).

- For any three objects $\boldsymbol{M}, \boldsymbol{M}^{\prime}$ and $\boldsymbol{M}^{\prime \prime}$ in eSLoc, we define the composition morphism - : eSLoc $\left(\boldsymbol{M}^{\prime}, \boldsymbol{M}^{\prime \prime}\right) \times \operatorname{eSLoc}\left(\boldsymbol{M}, \boldsymbol{M}^{\prime}\right) \rightarrow \operatorname{eSLoc}\left(\boldsymbol{M}, \boldsymbol{M}^{\prime \prime}\right)$ to be the natural transformation with components

$$
\operatorname{pt}_{n}:=\circ: \operatorname{eSLoc}\left(\boldsymbol{M}^{\prime}, \boldsymbol{M}^{\prime \prime}\right)\left(\mathrm{pt}_{n}\right) \times \operatorname{eSLoc}\left(\boldsymbol{M}, \boldsymbol{M}^{\prime}\right)\left(\mathrm{pt}_{n}\right) \longrightarrow \operatorname{eSLoc}\left(\boldsymbol{M}, \boldsymbol{M}^{\prime \prime}\right)\left(\mathrm{pt}_{n}\right),
$$

where $\circ$ is the composition of SMan/pt $n_{n}$-morphisms.

- For any object $\boldsymbol{M}$ in eSLoc, we define the identity on $\boldsymbol{M}$ morphism $\mathbf{1}: \mathfrak{I} \rightarrow \operatorname{eSLoc}(\boldsymbol{M}, \boldsymbol{M})$ to be the natural transformation with components

$$
\mathbf{1}_{\mathrm{pt}_{n}}: \mathrm{pt}=\mathfrak{I}\left(\mathrm{pt}_{n}\right) \longrightarrow \operatorname{eSLoc}(\boldsymbol{M}, \boldsymbol{M})\left(\mathrm{pt}_{n}\right), \star \rightsquigarrow \mathrm{id}_{\mathrm{pt}_{n} \times M}
$$

where $\mathrm{id}_{\mathrm{pt}_{n} \times M}$ is the identity SMan $/ \mathrm{pt}_{n}$-morphism.

Remark 6.7. Using Lemma 6.5 one can easily see that eSLoc is a SSet-category according to Definition A.1. Lemma 6.5 (iii) implies that the map of $\operatorname{sets} \operatorname{eSLoc}\left(\boldsymbol{M}, \boldsymbol{M}^{\prime}\right)\left(\lambda^{\text {op }}\right)$ is welldefined, i.e. that it has the claimed codomain, and Lemma 6.5 (i) implies that $\operatorname{eSLoc}\left(\boldsymbol{M}, \boldsymbol{M}^{\prime}\right)$ : $\mathrm{SPt}^{\mathrm{op}} \rightarrow$ Set is a functor. The composition $\bullet$ and identity $\mathbf{1}$ are natural transformations because of Lemma 6.5 (ii). Finally, the diagrams in Definition A.1 commute because of the associativity and identity property of the composition $\circ$ of SMan $/ \mathrm{pt}_{n}$-morphisms. 


\subsection{The SSet-functor $\mathfrak{e} \mathscr{O}: \mathrm{eSLoc}^{\mathrm{op}} \rightarrow \mathrm{eSVec}$}

Our next goal is to show that the ordinary global section functor $\mathscr{O}: \mathrm{SLoc}^{\mathrm{op}} \rightarrow \mathrm{SVec}$ can be promoted to a SSet-functor $\mathfrak{e} \mathscr{O}:$ eSLoc $^{\mathrm{op}} \rightarrow$ eSVec with values in the SSet-category eSVec of super-vector spaces. For defining the latter SSet-category we will first discuss how to promote super-vector spaces to (left) supermodules over Grassmann algebras. This procedure is known as "extension of ring of scalars" and discussed in detail in [Bou89, Chapter II.5].

Given any object $V$ in $\mathrm{SVec}$ and any object $\mathrm{pt}_{n}$ in SPt${ }^{\text {op }}$, we can consider the left $\Lambda_{n^{-}}$ supermodule $\Lambda_{n} \otimes V$. Given any two objects $V$ and $V^{\prime}$ in SVec, the set of $\Lambda_{n}$-SMod-morphisms $L: \Lambda_{n} \otimes V \rightarrow \Lambda_{n} \otimes V^{\prime}$ can be easily characterized.

Lemma 6.8. Let $V$ and $V^{\prime}$ be any two objects in $\mathrm{SVec}$ and $\mathrm{pt}_{n}$ any object in $\mathrm{SPt}^{\mathrm{op}}$. Then the map

$$
\begin{aligned}
\beta_{\mathrm{pt}_{n}}: \operatorname{Hom}_{\Lambda_{n}-\mathrm{SMod}}\left(\Lambda_{n} \otimes V, \Lambda_{n} \otimes V^{\prime}\right) & \longrightarrow \operatorname{Hom}_{\mathrm{SVec}}\left(V, \Lambda_{n} \otimes V^{\prime}\right) \\
\left(L: \Lambda_{n} \otimes V \rightarrow \Lambda_{n} \otimes V^{\prime}\right) & \longmapsto\left(L \circ\left(\eta_{n} \otimes \operatorname{id}_{V}\right): V \simeq \mathbb{R} \otimes V \rightarrow \Lambda_{n} \otimes V^{\prime}\right),
\end{aligned}
$$

is a bijection of sets, where $\eta_{n}: \mathbb{R} \rightarrow \Lambda_{n}$ denotes the unit in $\Lambda_{n}$.

Proof. The map $\beta_{\mathrm{pt}_{n}}$ is invertible by assigning to any SVec-morphism $K: V \rightarrow \Lambda_{n} \otimes V^{\prime}$ the $\Lambda_{n}$-SMod-morphism $\beta_{\mathrm{pt}_{n}}^{-1}(K): \Lambda_{n} \otimes V \rightarrow \Lambda_{n} \otimes V^{\prime}$ that is specified by $\beta_{\mathrm{pt}_{n}}^{-1}(K)(\zeta \otimes v):=$ $(\zeta \otimes \mathbb{1}) K(v)$, for all $\zeta \otimes v \in \Lambda_{n} \otimes V$, and $\mathbb{R}$-linear extension.

Given any two objects $V$ and $V^{\prime}$ in SVec and any $\mathrm{SPt}^{\mathrm{op}}$-morphism $\lambda^{\mathrm{op}}: \mathrm{pt}_{n} \rightarrow \mathrm{pt}_{m}$ (i.e. a SPt-morphism $\lambda: \mathrm{pt}_{m} \rightarrow \mathrm{pt}_{n}$ ) we can define a map of sets

$$
\operatorname{Hom}_{\mathrm{SVec}}\left(V, \Lambda_{n} \otimes V^{\prime}\right) \longrightarrow \operatorname{Hom}_{\mathrm{SVec}}\left(V, \Lambda_{m} \otimes V^{\prime}\right), \quad K \longmapsto\left(\lambda^{*} \otimes \operatorname{id}_{V^{\prime}}\right) \circ K .
$$

Using also Lemma 6.8 we obtain a map of sets

$$
\begin{aligned}
\lambda_{*}^{\mathrm{op}}: \operatorname{Hom}_{\Lambda_{n}-\mathrm{SMod}}\left(\Lambda_{n} \otimes V, \Lambda_{n} \otimes V^{\prime}\right) & \longrightarrow \operatorname{Hom}_{\Lambda_{m}-\mathrm{SMod}}\left(\Lambda_{m} \otimes V, \Lambda_{m} \otimes V^{\prime}\right), \\
L & \longmapsto \beta_{\mathrm{pt}_{m}}^{-1}\left(\left(\lambda^{*} \otimes \operatorname{id}_{V^{\prime}}\right) \circ \beta_{\mathrm{pt}_{n}}(L)\right) .
\end{aligned}
$$

The following properties can be easily derived from (6.17), see also Bou89. We therefore can omit the proof.

Lemma 6.9. (i) For any identity $\mathrm{SPt}^{\mathrm{op}}-$ morphism $\lambda^{\mathrm{op}}=\mathrm{id}_{\mathrm{pt}_{n}}: \mathrm{pt}_{n} \rightarrow \mathrm{pt}_{n}$ the map $\lambda_{*}^{\mathrm{op}}$ is the identity. For any two $\mathrm{SPt}^{\mathrm{op}}-$ morphisms $\lambda^{\mathrm{op}}: \mathrm{pt}_{n} \rightarrow \mathrm{pt}_{m}$ and $\lambda^{\prime \mathrm{op}}: \mathrm{pt}_{m} \rightarrow \mathrm{pt}_{l}$ we have that $\left(\lambda^{\prime \mathrm{op}} \circ \mathrm{op} \lambda^{\mathrm{op}}\right)_{*}=\lambda_{*}^{\mathrm{op}} \circ \lambda_{*}^{\mathrm{op}}$.

(ii) $\lambda_{*}^{\mathrm{op}}$ preserves identities and compositions, i.e.

$$
\lambda_{*}^{\mathrm{op}}\left(\operatorname{id}_{\Lambda_{n} \otimes V}\right)=\operatorname{id}_{\Lambda_{m} \otimes V}, \quad \lambda_{*}^{\mathrm{op}}\left(L^{\prime} \circ L\right)=\lambda_{*}^{\mathrm{op}}\left(L^{\prime}\right) \circ \lambda_{*}^{\mathrm{op}}(L),
$$

for all objects $V$ in SVec and all $\Lambda_{n}$-SMod-morphisms $L: \Lambda_{n} \otimes V \rightarrow \Lambda_{n} \otimes V^{\prime}$ and $L^{\prime}: \Lambda_{n} \otimes V^{\prime} \rightarrow \Lambda_{n} \otimes V^{\prime \prime}$.

(iii) $\lambda_{*}^{\text {op }}$ preserves isomorphisms, i.e. $L: \Lambda_{n} \otimes V \rightarrow \Lambda_{n} \otimes V^{\prime}$ is a $\Lambda_{n}$-SMod-isomorphism if and only if $\lambda_{*}^{\mathrm{op}}(L): \Lambda_{m} \otimes V \rightarrow \Lambda_{m} \otimes V^{\prime}$ is a $\Lambda_{m}$-SMod-isomorphism.

With these preparations we can now define the SSet-category eSVec.

Definition 6.10. The SSet-category eSVec is given by the following data:

- The objects are all objects $V$ in SVec. 
- For any two objects $V$ and $V^{\prime}$ in eSVec, the object of morphisms from $V$ to $V^{\prime}$ is given by the following functor $\operatorname{eSVec}\left(V, V^{\prime}\right): \mathrm{SPt}^{\mathrm{op}} \rightarrow$ Set: For any object $\mathrm{pt}_{n}$ in $\mathrm{SPt}^{\text {op }}$ we define eSVec $\left(V, V^{\prime}\right)\left(\mathrm{pt}_{n}\right)$ to be the set of all $\Lambda_{n}$-SMod-morphisms $L: \Lambda_{n} \otimes V \rightarrow \Lambda_{n} \otimes V^{\prime}$. For any $\mathrm{SPt}^{\mathrm{op}}$-morphism $\lambda^{\mathrm{op}}: \mathrm{pt}_{n} \rightarrow \mathrm{pt}_{m}$ we define the map of sets

$$
\operatorname{eSVec}\left(V, V^{\prime}\right)\left(\lambda^{\mathrm{op}}\right):=\lambda_{*}^{\mathrm{op}}: \operatorname{eSVec}\left(V, V^{\prime}\right)\left(\mathrm{pt}_{n}\right) \longrightarrow \operatorname{eSVec}\left(V, V^{\prime}\right)\left(\mathrm{pt}_{m}\right)
$$

where $\lambda_{*}^{\text {op }}$ is given in (6.17).

- For any three objects $V, V^{\prime}$ and $V^{\prime \prime}$ in eSVec, we define the composition morphism - : $\operatorname{eSVec}\left(V^{\prime}, V^{\prime \prime}\right) \times \operatorname{eSVec}\left(V, V^{\prime}\right) \rightarrow \operatorname{eSVec}\left(V, V^{\prime \prime}\right)$ to be the natural transformation with components

$$
\operatorname{pt}_{n}:=0: \operatorname{eSVec}\left(V^{\prime}, V^{\prime \prime}\right)\left(\mathrm{pt}_{n}\right) \times \operatorname{eSVec}\left(V, V^{\prime}\right)\left(\mathrm{pt}_{n}\right) \longrightarrow \operatorname{eSVec}\left(V, V^{\prime \prime}\right)\left(\mathrm{pt}_{n}\right),
$$

where $\circ$ is the composition of $\Lambda_{n}$-SMod-morphisms.

- For any object $V$ in eSVec, we define the identity on $V$ morphism $1: \mathfrak{I} \rightarrow \operatorname{eSVec}(V, V)$ to be the natural transformation with components

$$
\mathbf{1}_{\mathrm{pt}_{n}}: \mathrm{pt}=\mathfrak{I}\left(\mathrm{pt}_{n}\right) \longrightarrow \operatorname{eSVec}(V, V)\left(\mathrm{pt}_{n}\right), \quad \star \longmapsto \operatorname{id}_{\Lambda_{n} \otimes V},
$$

where $\operatorname{id}_{\Lambda_{n} \otimes V}$ is the identity $\Lambda_{n}$-SMod-morphism.

Remark 6.11. Let us briefly compare our category eSVec with the functorial formulation of super(linear) algebra used in [Sac08, Section 3], [Mol84, Section 2] and [Shv84]. In the latter approach, one considers module objects in the monoidal category SSet, i.e. functors $M$ : $\mathrm{SPt}{ }^{\mathrm{op}} \rightarrow$ Set such that all $M\left(\mathrm{pt}_{n}\right)$ carry module structures and all $M\left(\lambda^{\mathrm{op}}\right): M\left(\mathrm{pt}_{n}\right) \rightarrow M\left(\mathrm{pt}_{m}\right)$ are homomorphisms of modules. Together with appropriate morphisms in SSet, they form the category $\operatorname{Mod}(\mathrm{SSet})$. It is easy to see [Sac08, Section 3.4] that any object $V$ in SVec gives rise to an object $\bar{V}$ in $\operatorname{Mod}(\mathrm{SSet})$ (i.e. a functor) defined by

$$
\bar{V}\left(\mathrm{pt}_{n}\right):=\left(V \otimes \Lambda_{n}\right)_{0}, \quad \bar{V}\left(\lambda^{\mathrm{op}}: \mathrm{pt}_{n} \rightarrow \mathrm{pt}_{m}\right):=\left.\left(\mathrm{id}_{V} \otimes \lambda^{*}\right)\right|_{0} .
$$

Moreover, it can be shown [Sac08, Corollary 3.4.2] that the assignment $\div:$ SVec $\rightarrow$ Mod(SSet) defines a fully faithful functor; its image $\overline{\mathrm{SVec}}$ consists of representable modules. Comparing with Definition 6.10, we see that eSVec and $\overline{\mathrm{SVec}}$ have isomorphic classes of objects but our enriched category eSVec contains more morphisms. In fact, since - is full, we have $\operatorname{Hom}_{\overline{\mathrm{SVec}}}(\bar{V}, \bar{W}) \cong \operatorname{Hom}_{\mathrm{SVec}}(V, W) \cong \operatorname{eSVec}(V, W)\left(\mathrm{pt}_{0}\right)$. Thus, all additional information contained in $\operatorname{eSVec}(V, W)\left(\mathrm{pt}_{n}\right)$ for $n>0$ is not seen in $\overline{\mathrm{SVec}}$ and our definition provides a proper enrichment of the latter category. It may be possible to give a natural meaning to our enrichment constructions inside the functor categories Mod(SSet) (or SMod(SSet)), but this discussion is beyond the scope of the present publication.

We now can define a SSet-functor $\mathfrak{e} \mathscr{O}: \mathrm{eSLoc}^{\mathrm{op}} \rightarrow \mathrm{eSVec}$ as follows: To any object $M$ in eSLoc ${ }^{\text {op }}$ we assign the object $\mathfrak{e} \mathscr{O}(\boldsymbol{M}):=\mathscr{O}(\boldsymbol{M})$ in eSVec. To any two objects $\boldsymbol{M}$ and $\boldsymbol{M}^{\prime}$ in eSLoc ${ }^{\mathrm{op}}$ we assign the SSet-morphism $\mathfrak{e}_{\boldsymbol{M}, \boldsymbol{M}^{\prime}}: \operatorname{eSLoc}^{\mathrm{op}}\left(\boldsymbol{M}, \boldsymbol{M}^{\prime}\right) \rightarrow \operatorname{eSVec}\left(\mathscr{O}(\boldsymbol{M}), \mathscr{O}\left(\boldsymbol{M}^{\prime}\right)\right)$ given by the natural transformation (of functors from $\mathrm{SPt}^{\mathrm{op}}$ to $\mathrm{Set}$ ) with components

$$
\left(\mathfrak{e} \mathscr{O}_{\boldsymbol{M}, \boldsymbol{M}^{\prime}}\right)_{\mathrm{pt}_{n}}:\left(\chi: \mathrm{pt}_{n} \times M^{\prime} \rightarrow \mathrm{pt}_{n} \times M\right) \longmapsto\left(\chi^{*}: \Lambda_{n} \otimes \mathscr{O}(\boldsymbol{M}) \rightarrow \Lambda_{n} \otimes \mathscr{O}\left(\boldsymbol{M}^{\prime}\right)\right) .
$$

Naturality of these components is easily checked. We obtain

Proposition 6.12. The assignment $\mathfrak{e} \mathscr{O}: \mathrm{eSLoc}^{\mathrm{op}} \rightarrow \mathrm{eSVec}$ given above is a SSet-functor. 
Proof. We have to prove that this assignment is compatible with the composition and identity, see Definition A.3. Let $\mathrm{pt}_{n}$ be any object in $\mathrm{SPt}^{\mathrm{op}}$ and $\boldsymbol{M}, \boldsymbol{M}^{\prime}$ and $\boldsymbol{M}^{\prime \prime}$ any three objects in $\mathrm{eSLoc}^{\mathrm{op}}$. We obtain that, for all $\chi \in \mathrm{eSLoc}^{\mathrm{op}}\left(\boldsymbol{M}, \boldsymbol{M}^{\prime}\right)\left(\mathrm{pt}_{n}\right)$ and $\chi^{\prime} \in \mathrm{eSLoc}^{\mathrm{op}}\left(\boldsymbol{M}^{\prime}, \boldsymbol{M}^{\prime \prime}\right)\left(\mathrm{pt}_{n}\right)$,

$$
\begin{aligned}
\left(\mathfrak{e} \mathscr{O}_{\boldsymbol{M}, \boldsymbol{M}^{\prime \prime}}\right)_{\mathrm{pt}_{n}}\left(\chi^{\prime} \bullet \mathrm{pt}_{n} \mathrm{op}\right) & =\left(\mathfrak{e} \mathscr{O}_{\boldsymbol{M}, \boldsymbol{M}^{\prime \prime}}\right)_{\mathrm{pt}_{n}}\left(\chi \circ \chi^{\prime}\right)=\left(\chi \circ \chi^{\prime}\right)^{*}=\chi^{\prime *} \circ \chi^{*} \\
& =\left(\mathfrak{e} \mathscr{O}_{\boldsymbol{M}^{\prime}, \boldsymbol{M}^{\prime \prime}}\right)_{\mathrm{pt}_{n}}\left(\chi^{\prime}\right) \circ\left(\mathfrak{e} \mathscr{O}_{\boldsymbol{M}, \boldsymbol{M}^{\prime}}\right)_{\mathrm{pt}_{n}}(\chi) \\
& =\left(\mathfrak{e} \mathscr{O}_{\boldsymbol{M}^{\prime}, \boldsymbol{M}^{\prime \prime}}\right)_{\mathrm{pt}_{n}}\left(\chi^{\prime}\right) \bullet_{\mathrm{pt}_{n}}\left(\mathfrak{e} \mathscr{O}_{\boldsymbol{M}, \boldsymbol{M}^{\prime}}\right)_{\mathrm{pt}_{n}}(\chi),
\end{aligned}
$$

which proves compatibility with the composition. Compatibility with the identity is shown by

$$
\begin{aligned}
\left(\mathfrak{e} \mathscr{O}_{\boldsymbol{M}, \boldsymbol{M}}\right)_{\mathrm{pt}_{n}}\left(\mathbf{1}_{\mathrm{pt}_{n}}(\star)\right) & =\left(\mathfrak{e} \mathscr{O}_{\boldsymbol{M}, \boldsymbol{M}}\right)_{\mathrm{pt}_{n}}\left(\mathrm{id}_{\mathrm{pt}_{n} \times M}\right)=\left(\mathrm{id}_{\mathrm{pt}_{n} \times M}\right)^{*} \\
& =\operatorname{id}_{\Lambda_{n} \otimes \mathscr{O}(\boldsymbol{M})}=\mathbf{1}_{\mathrm{pt}_{n}}(\star),
\end{aligned}
$$

for all objects $M$ in eSLoc ${ }^{\text {op }}$.

Remark 6.13. As in the ordinary case, we can enlarge the morphisms in the SSet-category eSVec by replacing in Definition 6.10 all appearances of the sets $\operatorname{Hom}_{\Lambda_{n} \text {-SMod }}\left(\Lambda_{n} \otimes V, \Lambda_{n} \otimes V^{\prime}\right)$ of $\Lambda_{n}$-SMod-morphisms by the sets underlying the internal hom-objects $\underline{\operatorname{Hom}}_{\Lambda_{n}}\left(\Lambda_{n} \otimes V, \Lambda_{n} \otimes V^{\prime}\right)$ in the category $\Lambda_{n}$-SMod, i.e. all right $\Lambda_{n}$-linear maps $L: \Lambda_{n} \otimes V \rightarrow \Lambda_{n} \otimes V^{\prime}$. We denote the resulting SSet-category by $\underline{\mathrm{eSVec}}$ and note that there is an obvious SSet-functor eSVec $\rightarrow$ eSVec. Consequently, we can regard the SSet-functor $\mathfrak{e} \mathscr{O}:$ eSLoc $^{\mathrm{op}} \rightarrow$ eSVec also as a SSetfunctor (denoted with a slight abuse of notation by the same symbol) $\mathfrak{e} \mathscr{O}:$ eSLoc ${ }^{\mathrm{op}} \rightarrow \underline{\mathrm{eSVec}}$. As explained in Footnote 2, this generalization will be needed in Section 8 to describe 1|1dimensional examples (i.e. superparticles), which are somewhat peculiar.

\subsection{Structure of the SSet-natural transformations $\mathfrak{e} \mathscr{O} \Rightarrow \mathfrak{e} \mathscr{O}$}

In super-field theories, cf. Definition 4.2, we have described the dynamics by a suitable natural transformation $P: \mathscr{O} \Rightarrow \mathscr{O}$ of functors from SLoc ${ }^{\mathrm{op}}$ to $\underline{\mathrm{SVec}}$. In the enriched setting, we shall use suitable SSet-natural transformations $P: \mathfrak{e} \mathscr{O} \Rightarrow \mathfrak{e} \mathscr{O}$ of SSet-functors from eSLoc ${ }^{\text {op }}$ to eSVec. Recalling Definition A.5, such enriched natural transformations are given by assigning to every object $\boldsymbol{M}$ in eSLoc ${ }^{\mathrm{op}}$ a SSet-morphism $P_{\boldsymbol{M}}: \mathfrak{I} \rightarrow \underline{\mathrm{eSVec}}(\mathscr{O}(\boldsymbol{M}), \mathscr{O}(\boldsymbol{M}))$, such that the diagram given in this definition commutes. The SSet-morphisms $P_{\boldsymbol{M}}: \mathfrak{I} \rightarrow \underline{\operatorname{eSVec}}(\mathscr{O}(\boldsymbol{M}), \mathscr{O}(\boldsymbol{M}))$ are given by a natural transformations of functors from $\mathrm{SPt}^{\mathrm{op}}$ to Set, whose components are maps of sets that we denote by

$$
\begin{aligned}
\left(P_{\boldsymbol{M}}\right)_{\mathrm{pt}_{n}}: \mathrm{pt}=\mathfrak{I}\left(\mathrm{pt}_{n}\right) & \longrightarrow \underline{\mathrm{eSVec}}(\mathscr{O}(\boldsymbol{M}), \mathscr{O}(\boldsymbol{M}))\left(\mathrm{pt}_{n}\right), \\
\star & \longmapsto\left(P_{\boldsymbol{M}}\right)_{\mathrm{pt}_{n}}(\star)=: P_{\boldsymbol{M} / \mathrm{pt}_{n}} .
\end{aligned}
$$

Consequently, any SSet-morphism $P_{\boldsymbol{M}}: \mathfrak{I} \rightarrow \underline{\operatorname{eSVec}}(\mathscr{O}(\boldsymbol{M}), \mathscr{O}(\boldsymbol{M}))$ is specified by a collection of right $\Lambda_{n}$-linear maps $P_{\boldsymbol{M} / \mathrm{pt}_{n}}: \Lambda_{n} \otimes \mathscr{O}(\boldsymbol{M}) \rightarrow \Lambda_{n} \otimes \mathscr{O}(\boldsymbol{M})$, for all objects pt ${ }_{n}$ in SPt ${ }^{\text {op }}$, such that

$$
\lambda_{*}^{\mathrm{op}}\left(P_{\boldsymbol{M} / \mathrm{pt}_{n}}\right)=P_{\boldsymbol{M} / \mathrm{pt}_{m}},
$$

for all SPt ${ }^{\mathrm{op}}$-morphisms $\lambda^{\mathrm{op}}: \mathrm{pt}_{n} \rightarrow \mathrm{pt}_{m}$. Notice that, for all objects $\mathrm{pt}_{m}$ in SPt, there is the terminal SPt-morphism $\lambda: \mathrm{pt}_{m} \rightarrow \mathrm{pt}$ given by $\widetilde{\lambda}=\mathrm{id}_{\mathrm{pt}}$ and the unit $\lambda^{*}=\eta_{m}: \mathbb{R} \rightarrow \Lambda_{m}$ in $\Lambda_{m}$. As a consequence, the equation (6.26) applied to the terminal $\mathrm{SPt}^{\mathrm{op}}$-morphisms $\lambda^{\mathrm{op}}: \mathrm{pt} \rightarrow$ $\mathrm{pt}_{m}$ implies that any $P_{M / \mathrm{pt}_{m}}$ can be expressed in terms of the single right $\Lambda_{0}=\mathbb{R}$-linear map $P_{M / \mathrm{pt}}: \mathscr{O}(\boldsymbol{M}) \rightarrow \mathscr{O}(\boldsymbol{M})$ via

$$
\begin{aligned}
P_{\boldsymbol{M} / \mathrm{pt}_{m}}=\mathrm{id}_{\Lambda_{m}} \otimes P_{\boldsymbol{M} / \mathrm{pt}}: \Lambda_{m} \otimes \mathscr{O}(\boldsymbol{M}) & \longrightarrow \Lambda_{m} \otimes \mathscr{O}(\boldsymbol{M}) \\
\zeta \otimes F & \longrightarrow(-1)^{|\zeta|\left|P_{\boldsymbol{M} / \mathrm{pt}}\right|} \zeta \otimes P_{\boldsymbol{M} / \mathrm{pt}}(F) .
\end{aligned}
$$


The commutative diagram in Definition A.5 requires that

$$
P_{\boldsymbol{M}^{\prime} / \mathrm{pt}_{n}} \bullet \mathrm{pt}_{n}\left(\mathfrak{e} \mathscr{O}_{\boldsymbol{M}, \boldsymbol{M}^{\prime}}\right)_{\mathrm{pt}_{n}}(\chi)=\left(\mathfrak{e} \mathscr{O}_{\boldsymbol{M}, \boldsymbol{M}^{\prime}}\right)_{\mathrm{pt}_{n}}(\chi) \bullet \mathrm{pt}_{n} P_{\boldsymbol{M} / \mathrm{pt}_{n}},
$$

for all objects $\boldsymbol{M}$ and $\boldsymbol{M}^{\prime}$ in eSLoc ${ }^{\mathrm{op}}$, all objects $\mathrm{pt}_{n}$ in $\mathrm{SPt}^{\mathrm{op}}$ and all $\chi \in \mathrm{eSLoc}^{\mathrm{op}}\left(\boldsymbol{M}, \boldsymbol{M}^{\prime}\right)\left(\mathrm{pt}_{n}\right)$. Using (6.27), these conditions are equivalent to the commutative diagrams

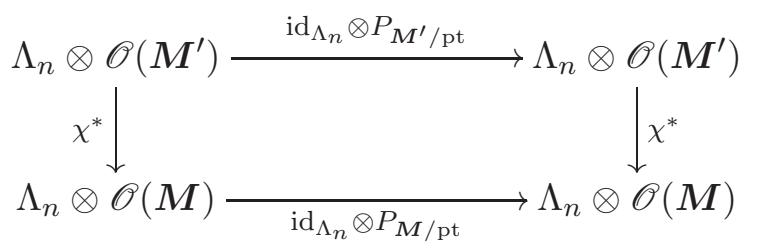

of right $\Lambda_{n}$-linear maps, for all objects $\boldsymbol{M}$ and $\boldsymbol{M}^{\prime}$ in eSLoc ${ }^{\mathrm{op}}$, all objects $\mathrm{pt}_{n}$ in $\mathrm{SPt}^{\mathrm{op}}$ and all $\chi \in \operatorname{eSLoc}^{\mathrm{op}}\left(\boldsymbol{M}^{\prime}, \boldsymbol{M}\right)\left(\mathrm{pt}_{n}\right)$. (For later convenience we have exchanged here $\boldsymbol{M}$ and $\boldsymbol{M}^{\prime}$.) We therefore have shown

Proposition 6.14. There is a bijective correspondence between

1. SSet-natural transformations $P: \mathfrak{e} \mathscr{O} \Rightarrow \mathfrak{e} \mathscr{O}$ of SSet-functors from eSLoc ${ }^{\mathrm{op}}$ to $\underline{\mathrm{eSVec}}$,

2. ordinary natural transformations $P: \mathscr{O} \Rightarrow \mathscr{O}$ of functors from $\mathrm{SLoc}^{\mathrm{op}}$ to $\underline{\mathrm{SVec}}$, such that the diagram

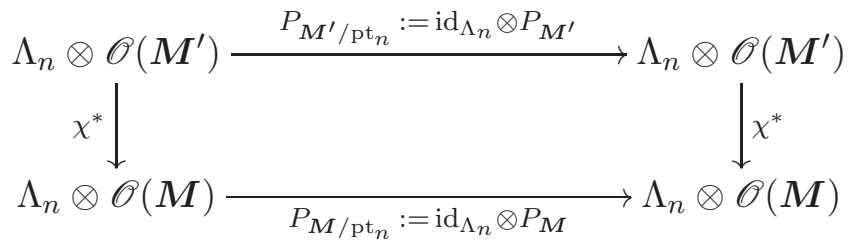

of right $\Lambda_{n}$-linear maps commutes, for all objects $\boldsymbol{M}$ and $\boldsymbol{M}^{\prime}$ in $\mathrm{eSLoc}^{\mathrm{op}}$, all objects $\mathrm{pt}_{n}$ in $\mathrm{SPt}^{\mathrm{op}}$ and all $\chi \in \mathrm{eSLoc}{ }^{\mathrm{op}}\left(\boldsymbol{M}^{\prime}, \boldsymbol{M}\right)\left(\mathrm{pt}_{n}\right)$.

\subsection{The definition}

With this preparation we can now give a simple definition of enriched super-field theories. In particular, using Proposition 6.14 we can define an enriched super-field theory to be a superfield theory (cf. Definition 4.2) together with extra conditions which ensure that the ordinary natural transformation $P: \mathscr{O} \Rightarrow \mathscr{O}$ extends to a SSet-natural transformation $P: \mathfrak{e} \mathscr{O} \Rightarrow \mathfrak{e} \mathscr{O}$.

Definition 6.15. An enriched super-field theory is a super-field theory according to Definition 4.2. such that the diagram (6.30) of right $\Lambda_{n}$-linear maps commutes, for all objects $\boldsymbol{M}$ and $\boldsymbol{M}^{\prime}$ in $\mathrm{eSLoc}^{\mathrm{op}}$, all objects $\mathrm{pt}_{n}$ in $\mathrm{SPt}^{\mathrm{op}}$ and all $\chi \in \mathrm{eSLoc}^{\mathrm{op}}\left(\boldsymbol{M}^{\prime}, \boldsymbol{M}\right)\left(\mathrm{pt}_{n}\right)$.

\section{Construction of enriched super-quantum field theories}

We show that given any enriched super-field theory according to Definition 6.15 one can construct a SSet-functor $\mathfrak{e A}:$ eSLoc $\rightarrow \mathrm{eS}^{*} \mathrm{Alg}$, i.e. an enriched super-QFT. As in Section 5 we decompose our construction into two steps: First, we construct a SSet-functor $\mathfrak{e} \mathfrak{L}:$ eSLoc $\rightarrow$ eX which describes the enriched classical theory. Second, we construct a SSet-functor $\mathfrak{e} \mathfrak{Q}: \mathrm{eX} \rightarrow$ eS*Alg describing the enriched quantization. We shall also study properties of the enriched functors and establish a connection between the enriched super-field theory and the enriched super-QFT by constructing an enriched locally covariant quantum field. In contrast to ordinary super-QFTs, our enriched approach captures also supersymmetry transformations. It should be emphasized that the SSet-categories eSLoc, eX and eS*Alg are defined using $\mathbb{Z}_{2}$-parity preserving morphisms. The appearance of supersymmetry transformations in the enriched setting is due to the higher superpoints of the morphism supersets in eSLoc, eX and eS*Alg. 


\subsection{The SSet-functor $\mathfrak{e} \mathscr{O}_{\mathrm{c}}:$ eSLoc $\rightarrow$ eSVec}

As a preparatory step we construct a SSet-functor $\mathfrak{e} \mathscr{O}_{c}:$ eSLoc $\rightarrow e S V e c$ that assigns to objects $\boldsymbol{M}$ in eSLoc the super-vector spaces of compactly supported sections $\mathfrak{e} \mathscr{O}_{\mathrm{c}}(\boldsymbol{M}):=\mathscr{O}_{\mathrm{c}}(\boldsymbol{M})$. We assign to any two objects $\boldsymbol{M}$ and $\boldsymbol{M}^{\prime}$ in eSLoc the SSet-morphism $\mathfrak{e} \mathscr{O}_{\mathrm{c} \boldsymbol{M}, \boldsymbol{M}^{\prime}}: \operatorname{eSLoc}\left(\boldsymbol{M}, \boldsymbol{M}^{\prime}\right) \rightarrow$ $\operatorname{eSVec}\left(\mathscr{O}_{\mathrm{c}}(\boldsymbol{M}), \mathscr{O}_{\mathrm{c}}\left(\boldsymbol{M}^{\prime}\right)\right)$ given by the natural transformation (of functors from $\mathrm{SPt}^{\mathrm{op}}$ to Set) with components

$$
\left(\mathfrak{e} \mathscr{O}_{\mathrm{c} M, \boldsymbol{M}^{\prime}}\right)_{\mathrm{pt}_{n}}:\left(\chi: \mathrm{pt}_{n} \times M \rightarrow \mathrm{pt}_{n} \times M^{\prime}\right) \longmapsto\left(\chi_{*}: \Lambda_{n} \otimes \mathscr{O}_{\mathrm{c}}(\boldsymbol{M}) \rightarrow \Lambda_{n} \otimes \mathscr{O}_{\mathrm{c}}\left(\boldsymbol{M}^{\prime}\right)\right),
$$

where $\chi_{*}$ denotes the push-forward of compactly supported sections of the structure sheaf $\mathscr{O}_{\mathrm{pt}_{n} \times M}$ (cf. (5.5)), which exists as a consequence of the conditions 1.) and 2.) in Definition 6.6. Because of functoriality of the push-forwards, i.e. $\left(\operatorname{id}_{\mathrm{pt}_{n} \times M}\right)_{*}=\operatorname{id}_{\Lambda_{n} \otimes \mathscr{O}_{\mathrm{c}}(\boldsymbol{M})}$ and $\left(\chi^{\prime} \circ \chi\right)_{*}=$ $\chi_{*}^{\prime} \circ \chi_{*}$ as in Lemma 5.1 (iii), we have shown

Proposition 7.1. The assignment $\mathfrak{e} \mathscr{O}_{\mathrm{c}}: \mathrm{eSLoc} \rightarrow \mathrm{eSVec}$ given above is a SSet-functor.

Let $\boldsymbol{M}=(M, \Omega, E)$ be any object in eSLoc and $\mathrm{pt}_{n}$ any object in $\mathrm{SPt}^{\mathrm{op}}$. Making use of the $\mathrm{pt}_{n}$-relative differential geometry on $M / \mathrm{pt}_{n}$, together with the $\mathrm{pt}_{n}$-relative supervielbein $\mathbb{1} \otimes E \in \Omega^{1}\left(M / \mathrm{pt}_{n}, \mathfrak{t}\right)$, we can define a $\mathrm{pt}_{n}$-relative version of the pairing (3.11) by

$$
\begin{aligned}
\langle\cdot, \cdot\rangle_{M / \mathrm{pt}_{n}}:\left(\Lambda_{n} \otimes \mathscr{O}_{\mathrm{c}}(\boldsymbol{M})\right) \otimes_{\Lambda_{n}}\left(\Lambda_{n} \otimes \mathscr{O}_{\mathrm{c}}(\boldsymbol{M})\right) & \longrightarrow \Lambda_{n}, \\
H_{1} \otimes_{\Lambda_{n}} H_{2} & \longmapsto \int_{M / \mathrm{pt}_{n}} \operatorname{Ber}(\mathbb{1} \otimes E) H_{1} H_{2},
\end{aligned}
$$

where $\int_{M / \mathrm{pt}_{n}}$ is the $\mathrm{pt}_{n^{-}}$-relative Berezin integral, see e.g. [DM99, §3.10]. Explicitly, the $\mathrm{pt}_{n^{-}}$ relative pairing reads as

$$
\begin{aligned}
\left\langle\zeta_{1} \otimes F_{1}, \zeta_{2} \otimes F_{2}\right\rangle_{M / \mathrm{pt}_{n}} & =(-1)^{\left(\left|\zeta_{1}\right|+\left|\zeta_{2}\right|\right)|\operatorname{Ber}(E)|}(-1)^{\left|\zeta_{2}\right|\left|F_{1}\right|} \zeta_{1} \zeta_{2} \int_{M} \operatorname{Ber}(E) F_{1} F_{2} \\
& =(-1)^{\left(\left|\zeta_{1}\right|+\left|\zeta_{2}\right|\right)\left|P_{M}\right|}(-1)^{\left|\zeta_{2}\right|\left|F_{1}\right|} \zeta_{1} \zeta_{2}\left\langle F_{1}, F_{2}\right\rangle_{M},
\end{aligned}
$$

for all homogeneous $\zeta_{1}, \zeta_{2} \in \Lambda_{n}$ and $F_{1}, F_{2} \in \mathscr{O}_{\mathrm{c}}(\boldsymbol{M})$, i.e. it is given by $\Lambda_{n}$-superlinear extension of the pairing on $\mathscr{O}_{c}(\boldsymbol{M})$. Here, we have moreover used that by assumption $|\operatorname{Ber}(E)|=\left|P_{M}\right|$. Notice that (7.2) can be extended to all $H_{1}, H_{2} \in \Lambda_{n} \otimes \mathscr{O}(\boldsymbol{M})$ with compactly overlapping support.

Lemma 7.2. Let $\boldsymbol{M}$ and $\boldsymbol{M}^{\prime}$ be any two objects in $\mathrm{eSLoc}$ and $\mathrm{pt}_{n}$ any object in $\mathrm{SPt}^{\mathrm{op}}$. Then for any $\chi \in \operatorname{eSLoc}\left(\boldsymbol{M}, \boldsymbol{M}^{\prime}\right)\left(\mathrm{pt}_{n}\right)$ the following properties hold true:

(i) $\chi^{*} \circ \chi_{*}=\operatorname{id}_{\Lambda_{n} \otimes \mathscr{O}_{\mathrm{c}}(\boldsymbol{M})}$ and $\chi_{*} \circ \chi^{*}(H)=H$, for all $H \in \Lambda_{n} \otimes \mathscr{O}_{\mathrm{c}}\left(\boldsymbol{M}^{\prime}\right)$ such that $\operatorname{supp}(H) \subseteq$ $\widetilde{\chi}(\widetilde{M})$.

(ii) $\left\langle H_{1}, \chi_{*}\left(H_{2}\right)\right\rangle_{\boldsymbol{M}^{\prime} / \mathrm{pt}_{n}}=\left\langle\chi^{*}\left(H_{1}\right), H_{2}\right\rangle_{\boldsymbol{M} / \mathrm{pt}_{n}}$, for all $H_{1} \in \Lambda_{n} \otimes \mathscr{O}\left(\boldsymbol{M}^{\prime}\right)$ and $H_{2} \in \Lambda_{n} \otimes$ $\mathscr{O}_{\mathrm{c}}(\boldsymbol{M})$.

Proof. The proof of item (i) is as in Lemma 5.1 (i). In the proof of item (ii) one follows the same steps as in Lemma 5.1 (ii), but uses instead of the usual transformation formula (2.25) its relative version for the relative Berezin integral, see [DM99, §3.10].

\subsection{Enriched properties of the super-Green's operators}

Let us fix any enriched super-field theory according to Definition 6.15. As a consequence of $P_{M}$ being formally super-self adjoint (cf. (4.2)) one easily checks by using (17.3) that the $\mathrm{pt}_{n}$-relative super-differential operator $P_{\boldsymbol{M} / \mathrm{pt}_{n}}:=\operatorname{id}_{\Lambda_{n}} \otimes P_{\boldsymbol{M}}: \Lambda_{n} \otimes \mathscr{O}(\boldsymbol{M}) \rightarrow \Lambda_{n} \otimes \mathscr{O}(\boldsymbol{M})$ satisfies

$$
\left\langle H_{1}, P_{\boldsymbol{M} / \mathrm{pt}_{n}}\left(H_{2}\right)\right\rangle_{\boldsymbol{M} / \mathrm{pt}_{n}}=(-1)^{\left|H_{1}\right|\left|P_{M}\right|}\left\langle P_{\boldsymbol{M} / \mathrm{pt}_{n}}\left(H_{1}\right), H_{2}\right\rangle_{\boldsymbol{M} / \mathrm{pt}_{n}},
$$


for any object $\mathrm{pt}_{n}$ in $\mathrm{SPt}^{\mathrm{op}}$ and all homogeneous $H_{1}, H_{2} \in \Lambda_{n} \otimes \mathscr{O}(\boldsymbol{M})$ with compactly overlapping support. The right $\Lambda_{n}$-linear maps $G_{\boldsymbol{M} / \mathrm{pt}_{n}}^{ \pm}:=\mathrm{id}_{\Lambda_{n}} \otimes G_{\boldsymbol{M}}^{ \pm}: \Lambda_{n} \otimes \mathscr{O}_{\mathrm{c}}(\boldsymbol{M}) \rightarrow \Lambda_{n} \otimes \mathscr{O}(\boldsymbol{M})$ are easily seen to be retarded/advanced super-Green's operators for $P_{\boldsymbol{M} / \mathrm{pt}_{n}}$. We also define $G_{\boldsymbol{M} / \mathrm{pt}_{n}}:=G_{\boldsymbol{M} / \mathrm{pt}_{n}}^{+}-G_{\boldsymbol{M} / \mathrm{pt}_{n}}^{-}: \Lambda_{n} \otimes \mathscr{O}_{\mathrm{c}}(\boldsymbol{M}) \rightarrow \Lambda_{n} \otimes \mathscr{O}_{\mathrm{sc}}(\boldsymbol{M})$. Our statements in Subsection 5.2 easily generalize to the $\mathrm{pt}_{n}$-relative setting since all the proofs are algebraic and only use the properties of super-Green's operators. We summarize without repeating the proofs the main properties which are used in the next subsections.

Lemma 7.3. (i) Let $\boldsymbol{M}$ be any object in $\mathrm{eSLoc}$ and $\mathrm{pt}_{n}$ any object in $\mathrm{SPt}^{\mathrm{op}}$. Then

$$
\left\langle H_{1}, G_{\boldsymbol{M} / \mathrm{pt}_{n}}^{ \pm}\left(H_{2}\right)\right\rangle_{\boldsymbol{M} / \mathrm{pt}_{n}}=(-1)^{\left(\left|H_{1}\right|+\left|P_{M}\right|\right)\left|P_{M}\right|}\left\langle G_{\boldsymbol{M} / \mathrm{pt}_{n}}^{\mp}\left(H_{1}\right), H_{2}\right\rangle_{\boldsymbol{M} / \mathrm{pt}_{n}},
$$

for all homogeneous $H_{1}, H_{2} \in \Lambda_{n} \otimes \mathscr{O}_{\mathrm{c}}(\boldsymbol{M})$.

(ii) Let $\boldsymbol{M}$ be any object in $\mathrm{eSLoc}$ and $\mathrm{pt}_{n}$ any object in $\mathrm{SPt}^{\mathrm{op}}$. Then the sequence of right $\Lambda_{n}$-linear maps

$$
0 \rightarrow \Lambda_{n} \otimes \mathscr{O}_{\mathrm{c}}(\boldsymbol{M}) \stackrel{P_{M / \mathrm{pt}_{n}}}{\longrightarrow} \Lambda_{n} \otimes \mathscr{O}_{\mathrm{c}}(\boldsymbol{M}) \stackrel{G_{\boldsymbol{M} / \mathrm{pt}_{n}}}{\longrightarrow} \Lambda_{n} \otimes \mathscr{O}_{\mathrm{sc}}(\boldsymbol{M}) \stackrel{P_{M / \mathrm{pt}_{n}}}{\longrightarrow} \Lambda_{n} \otimes \mathscr{O}_{\mathrm{sc}}(\boldsymbol{M})
$$

is a complex which is exact everywhere.

(iii) Let $\mathrm{pt}_{n}$ be any object in $\operatorname{SPt}{ }^{\text {op }}$ and $\chi \in \operatorname{eSLoc}\left(\boldsymbol{M}, \boldsymbol{M}^{\prime}\right)\left(\mathrm{pt}_{n}\right)$. Then

$$
G_{M / \mathrm{pt}_{n}}^{ \pm}=\chi^{*} \circ G_{\boldsymbol{M}^{\prime} / \mathrm{pt}_{n}}^{ \pm} \circ \chi_{*} .
$$

\subsection{The SSet-functor $\mathfrak{e} \mathfrak{L}:$ eSLoc $\rightarrow$ eX}

Given any object $\boldsymbol{V}=(V, \tau)$ in $\mathrm{X}$ and any object $\mathrm{pt}_{n}$ in $\mathrm{SPt}^{\mathrm{op}}$, we can consider the object $\Lambda_{n} \otimes V$ in $\Lambda_{n}$-SMod and define a $\Lambda_{n}$-SMod-morphism

$$
\begin{aligned}
\tau_{\mathrm{pt}_{n}}:\left(\Lambda_{n} \otimes V\right) \otimes_{\Lambda_{n}}\left(\Lambda_{n} \otimes V\right) & \longrightarrow \Lambda_{n}, \\
\left(\zeta_{1} \otimes v_{1}\right) \otimes_{\Lambda_{n}}\left(\zeta_{2} \otimes v_{2}\right) & \longmapsto(-1)^{\left|\zeta_{2}\right|\left|v_{1}\right|} \zeta_{1} \zeta_{2} \tau\left(v_{1}, v_{2}\right) .
\end{aligned}
$$

Let us now enrich the category $\mathrm{X}$ given in Definition 5.7

Definition 7.4. The SSet-category eX is given by the following data:

- The objects are all objects $\boldsymbol{V}=(V, \tau)$ in $\mathrm{X}$.

- For any two objects $\boldsymbol{V}$ and $\boldsymbol{V}^{\prime}$ in eX, the object of morphisms from $\boldsymbol{V}$ to $\boldsymbol{V}^{\prime}$ is the following functor eX $\left(\boldsymbol{V}, \boldsymbol{V}^{\prime}\right): \mathrm{SPt}^{\mathrm{op}} \rightarrow$ Set: For any object $\mathrm{pt}_{n}$ in $\mathrm{SPt}^{\mathrm{op}}$ we define $\mathrm{eX}\left(\boldsymbol{V}, \boldsymbol{V}^{\prime}\right)\left(\mathrm{pt}_{n}\right)$ to be the set of all $\Lambda_{n}$-SMod-morphisms $L: \Lambda_{n} \otimes V \rightarrow \Lambda_{n} \otimes V^{\prime}$ satisfying $\tau_{\mathrm{pt}_{n}}^{\prime} \circ\left(L \otimes_{\Lambda_{n}} L\right)=$ $\tau_{\mathrm{pt}_{n}}$. For any $\mathrm{SPt}^{\mathrm{op}}-$ morphism $\lambda^{\mathrm{op}}: \mathrm{pt}_{n} \rightarrow \mathrm{pt}_{m}$ we define the map of sets

$$
\mathrm{eX}\left(\boldsymbol{V}, \boldsymbol{V}^{\prime}\right)\left(\lambda^{\mathrm{op}}\right):=\lambda_{*}^{\mathrm{op}}: \operatorname{eX}\left(\boldsymbol{V}, \boldsymbol{V}^{\prime}\right)\left(\mathrm{pt}_{n}\right) \longrightarrow \mathrm{eX}\left(\boldsymbol{V}, \boldsymbol{V}^{\prime}\right)\left(\mathrm{pt}_{m}\right),
$$

where $\lambda_{*}^{\text {op }}$ is given in (6.17).

- The composition and identity morphisms are defined as in the SSet-category eSVec, see Definition 6.10. 
We can now define the SSet-functor $\mathfrak{e} \mathfrak{L}: \mathrm{eSLoc} \rightarrow \mathrm{eX}$ as follows: To any object $\boldsymbol{M}$ in eSLoc we assign the object $\mathfrak{e} \mathfrak{L}(\boldsymbol{M}):=\mathfrak{L}(\boldsymbol{M})$ in eX that is given in (5.22). To any two objects $\boldsymbol{M}$ and $\boldsymbol{M}^{\prime}$ in eSLoc we assign the SSet-morphism $\mathfrak{e} \mathfrak{L}_{\boldsymbol{M}, \boldsymbol{M}^{\prime}}: \operatorname{eSLoc}\left(\boldsymbol{M}, \boldsymbol{M}^{\prime}\right) \rightarrow \operatorname{eX}\left(\mathfrak{L}(\boldsymbol{M}), \mathfrak{L}\left(\boldsymbol{M}^{\prime}\right)\right)$ given by the natural transformation (of functors from $\mathrm{SPt}^{\mathrm{op}}$ to Set) with components

$$
\left(\mathfrak{e} \mathfrak{L}_{M, M^{\prime}}\right)_{\mathrm{pt}_{n}}(\chi): \Lambda_{n} \otimes \mathfrak{L}(\boldsymbol{M}) \longrightarrow \Lambda_{n} \otimes \mathfrak{L}\left(\boldsymbol{M}^{\prime}\right), \quad[H] \longmapsto\left[\chi_{*}(H)\right],
$$

for all $\chi \in \operatorname{eSLoc}\left(\boldsymbol{M}, \boldsymbol{M}^{\prime}\right)\left(\mathrm{pt}_{n}\right)$. Notice that (7.10) is well-defined since

$$
\begin{aligned}
\chi_{*}\left(\zeta \otimes P_{M}(F)\right) & =(-1)^{\left|P_{M}\right||\zeta|} \chi_{*} \circ P_{M / \mathrm{pt}_{n}} \circ \chi^{*} \circ \chi_{*}(\zeta \otimes F) \\
& =(-1)^{\left|P_{M}\right||\zeta|} \chi_{*} \circ \chi^{*} \circ P_{\boldsymbol{M}^{\prime} / \mathrm{pt}_{n}} \circ \chi_{*}(\zeta \otimes F) \\
& =(-1)^{\left|P_{M}\right||\zeta|} P_{\boldsymbol{M}^{\prime} / \mathrm{pt}_{n}} \circ \chi_{*}(\zeta \otimes F) \in \Lambda_{n} \otimes P_{\boldsymbol{M}^{\prime}}\left(\mathscr{O}_{\mathbf{C}}\left(\boldsymbol{M}^{\prime}\right)\right),
\end{aligned}
$$

where in the first and third equality we have used Lemma 7.2 (i) and in the second equality the commutative diagram (6.30). Moreover, (7.10) defines an element in eX $\left(\mathfrak{L}(\boldsymbol{M}), \mathfrak{L}\left(\boldsymbol{M}^{\prime}\right)\right)\left(\mathrm{pt}_{n}\right)$ because of the equality

$$
\tau_{M / \mathrm{pt}_{n}}\left(\left[H_{1}\right],\left[H_{2}\right]\right)=\left\langle G_{\boldsymbol{M} / \mathrm{pt}_{n}}\left(H_{1}\right), H_{2}\right\rangle_{\boldsymbol{M} / \mathrm{pt}_{n}},
$$

for all $\left[H_{1}\right],\left[H_{2}\right] \in \Lambda_{n} \otimes \mathfrak{L}(\boldsymbol{M})$ and all objects $\boldsymbol{M}$ in eSLoc, from which it follows that $\tau_{\boldsymbol{M}^{\prime} / \mathrm{pt}_{n}} \circ$ $\left(\left(\mathfrak{e} \mathfrak{L}_{\boldsymbol{M}, \boldsymbol{M}^{\prime}}\right)_{\mathrm{pt}_{n}}(\chi) \otimes_{\Lambda_{n}}\left(\mathfrak{e} \mathfrak{L}_{\boldsymbol{M}, \boldsymbol{M}^{\prime}}\right)_{\mathrm{pt}_{n}}(\chi)\right)=\tau_{\boldsymbol{M} / \mathrm{pt}_{n}}$, for all $\chi \in \operatorname{eSLoc}\left(\boldsymbol{M}, \boldsymbol{M}^{\prime}\right)\left(\mathrm{pt}_{n}\right)$; indeed,

$$
\begin{aligned}
\tau_{M^{\prime} / \mathrm{pt}_{n}}\left(\left[\chi_{*}\left(H_{1}\right)\right],\left[\chi_{*}\left(H_{2}\right)\right]\right) & =\left\langle G_{\boldsymbol{M}^{\prime} / \mathrm{pt}_{n}} \circ \chi_{*}\left(H_{1}\right), \chi_{*}\left(H_{2}\right)\right\rangle_{\boldsymbol{M}^{\prime} / \mathrm{pt}_{n}} \\
& =\left\langle\chi^{*} \circ G_{\boldsymbol{M}^{\prime} / \mathrm{pt}_{n}} \circ \chi_{*}\left(H_{1}\right), H_{2}\right\rangle_{\boldsymbol{M} / \mathrm{pt}_{n}} \\
& =\left\langle G_{\boldsymbol{M} / \mathrm{pt}_{n}}\left(H_{1}\right), H_{2}\right\rangle_{\boldsymbol{M} / \mathrm{pt}_{n}}=\tau_{\boldsymbol{M} / \mathrm{pt}_{n}}\left(\left[H_{1}\right],\left[H_{2}\right]\right),
\end{aligned}
$$

for all $\left[H_{1}\right],\left[H_{2}\right] \in \Lambda_{n} \otimes \mathfrak{L}(\boldsymbol{M})$, where in the second equality we have used Lemma 7.2 (ii) and in the third equality Lemma 7.3 (iii). In summary, we have shown

Proposition 7.5. The assignment $\mathfrak{e} \mathfrak{L}: \mathrm{eSLoc} \rightarrow \mathrm{eX}$ given above is a SSet-functor.

We finish this subsection by observing that the SSet-functor $\mathfrak{e} \mathfrak{L}:$ eSLoc $\rightarrow$ eX satisfies an enriched version of the properties in Theorem 5.9 for ordinary super-field theories, which can be proven in exactly the same way:

Theorem 7.6. For any enriched super-field theory according to Definition 6.15 the associated SSet-functor $\mathfrak{e} \mathfrak{L}:$ eSLoc $\rightarrow$ eX satisfies the following properties, for all objects $\mathrm{pt}_{n}$ in $\mathrm{SPt}^{\mathrm{op}}$ :

- Enriched locality: For any $\chi \in \operatorname{eSLoc}\left(\boldsymbol{M}, \boldsymbol{M}^{\prime}\right)\left(\mathrm{pt}_{n}\right)$, we have that $\left(\mathfrak{e} \mathfrak{L}_{\boldsymbol{M}, \boldsymbol{M}^{\prime}}\right)_{\mathrm{pt}_{n}}(\chi) \in$ $\mathrm{eX}\left(\mathfrak{L}(\boldsymbol{M}), \mathfrak{L}\left(\boldsymbol{M}^{\prime}\right)\right)\left(\mathrm{pt}_{n}\right)$ is monic.

- Enriched super-causality: Given $\chi_{1} \in \operatorname{eSLoc}\left(\boldsymbol{M}_{1}, \boldsymbol{M}\right)\left(\mathrm{pt}_{n}\right)$ and $\chi_{2} \in \operatorname{eSLoc}\left(\boldsymbol{M}_{2}, \boldsymbol{M}\right)\left(\mathrm{pt}_{n}\right)$,

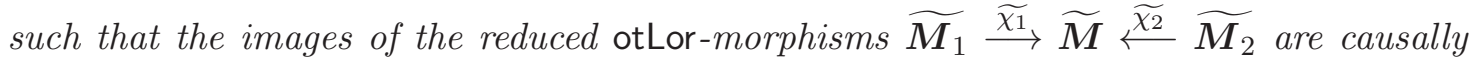
disjoint, then

$$
\tau_{\boldsymbol{M} / \mathrm{pt}_{n}}\left(\left(\mathfrak{e} \mathfrak{L}_{\boldsymbol{M}_{1}, \boldsymbol{M}}\right)_{\mathrm{pt}_{n}}\left(\chi_{1}\right)\left(\Lambda_{n} \otimes \mathfrak{L}\left(\boldsymbol{M}_{1}\right)\right),\left(\mathfrak{e} \mathfrak{L}_{\boldsymbol{M}_{2}, \boldsymbol{M}}\right)_{\mathrm{pt}_{n}}\left(\chi_{2}\right)\left(\Lambda_{n} \otimes \mathfrak{L}\left(\boldsymbol{M}_{2}\right)\right)\right)=\{0\} .
$$

- Enriched time-slice axiom: Given any $\chi \in \operatorname{eSLoc}\left(\boldsymbol{M}, \boldsymbol{M}^{\prime}\right)\left(\mathrm{pt}_{n}\right)$ such that $\tilde{\chi}: \widetilde{\boldsymbol{M}} \rightarrow \widetilde{\boldsymbol{M}^{\prime}}$ is Cauchy, then $\left(\mathfrak{e} \mathfrak{L}_{\boldsymbol{M}, \boldsymbol{M}^{\prime}}\right)_{\mathrm{pt}_{n}}(\chi) \in \operatorname{eX}\left(\mathfrak{L}(\boldsymbol{M}), \mathfrak{L}\left(\boldsymbol{M}^{\prime}\right)\right)\left(\mathrm{pt}_{n}\right)$ is an isomorphism. 


\subsection{The SSet-quantization functor $\mathfrak{e Q}: \mathrm{eX} \rightarrow \mathrm{eS}^{*} \mathrm{Alg}$}

We define an enriched version of the category S*Alg using "extension of scalars" for algebras (cf. [Bou89, Chapter III.1.5] for the general concept) and adapt the results obtained in Subsection 6.3 to the category of super-*-algebras. For this let us denote by $\Lambda_{n}^{\mathbb{C}}:=\Lambda_{n} \otimes \mathbb{C}$ the complexification of the Grassmann algebra, for all $n \in \mathbb{N}^{0}$, and notice that $\Lambda_{n}^{\mathbb{C}}$ is an object in $\mathrm{S}^{*}$ Alg when equipped with the superinvolution $*:=\operatorname{id}_{\Lambda_{n}} \otimes-: \Lambda_{n}^{\mathbb{C}} \rightarrow \Lambda_{n}^{\mathbb{C}}$. We shall denote the product in $\Lambda_{n}^{\mathbb{C}}$ by $\mu_{n}^{\mathbb{C}}$ and the unit by $\eta_{n}^{\mathbb{C}}$. Let $A$ and $A^{\prime}$ be any two objects in $\mathrm{S}^{*} A$ Ig and $\mathrm{pt}_{n}$ any object in $\mathrm{SPt}^{\mathrm{op}}$. A S*Alg-morphism $\kappa: \Lambda_{n}^{\mathbb{C}} \otimes_{\mathbb{C}} A \rightarrow \Lambda_{n}^{\mathbb{C}} \otimes_{\mathbb{C}} A^{\prime}$ is called a $\Lambda_{n}^{\mathbb{C}}$-relative $\mathrm{S}^{*} \mathrm{Alg}$-morphism (in short $\Lambda_{n}^{\mathbb{C}}$-S*Alg-morphism) provided that $\kappa\left(\zeta \otimes_{\mathbb{C}} \mathbb{1}\right)=\zeta \otimes_{\mathbb{C}} \mathbb{1}$ for all $\zeta \in \Lambda_{n}^{\mathbb{C}}$, i.e. $\kappa$ is $\Lambda_{n}$-superlinear. Notice that the identity $\operatorname{id}_{\Lambda_{n}^{\mathbb{C}} \otimes \mathbb{C} A}$ is a $\Lambda_{n}^{\mathbb{C}}$-S*Alg-morphism and that any two $\Lambda_{n}^{\mathbb{C}}$-S*Alg-morphisms $\kappa: \Lambda_{n}^{\mathbb{C}} \otimes_{\mathbb{C}} A \rightarrow \Lambda_{n}^{\mathbb{C}} \otimes_{\mathbb{C}} A^{\prime}$ and $\kappa^{\prime}: \Lambda_{n}^{\mathbb{C}} \otimes_{\mathbb{C}} A^{\prime} \rightarrow \Lambda_{n}^{\mathbb{C}} \otimes_{\mathbb{C}} A^{\prime \prime}$ can be composed, i.e. $\kappa^{\prime} \circ \kappa: \Lambda_{n}^{\mathbb{C}} \otimes_{\mathbb{C}} A \rightarrow \Lambda_{n}^{\mathbb{C}} \otimes_{\mathbb{C}} A^{\prime \prime}$ is a $\Lambda_{n}^{\mathbb{C}}$-S*Alg-morphism. In analogy to Lemma 6.8, the $\Lambda_{n}^{\mathbb{C}}$-S*Alg-morphisms $\kappa: \Lambda_{n}^{\mathbb{C}} \otimes_{\mathbb{C}} A \rightarrow \Lambda_{n}^{\mathbb{C}} \otimes_{\mathbb{C}} A^{\prime}$ can be easily characterized.

Lemma 7.7. Let $A$ and $A^{\prime}$ be any two objects in $\mathrm{S}^{*} \mathrm{Alg}$ and $\mathrm{pt}_{n}$ any object in $\mathrm{SPt}^{\mathrm{op}}$. Then the map

$$
\begin{aligned}
\gamma_{\mathrm{pt}_{n}}: \operatorname{Hom}_{\Lambda_{n}^{\mathbb{C}}-\mathrm{S}^{*} \mathrm{Alg}}\left(\Lambda_{n}^{\mathbb{C}} \otimes_{\mathbb{C}} A, \Lambda_{n}^{\mathbb{C}} \otimes_{\mathbb{C}} A^{\prime}\right) & \longrightarrow \operatorname{Hom}_{\mathrm{S}^{*} \operatorname{Alg}}\left(A, \Lambda_{n}^{\mathbb{C}} \otimes_{\mathbb{C}} A^{\prime}\right), \\
\left(\kappa: \Lambda_{n}^{\mathbb{C}} \otimes_{\mathbb{C}} A \rightarrow \Lambda_{\mathbb{C}}^{n} \otimes_{\mathbb{C}} A^{\prime}\right) & \longmapsto\left(\kappa \circ\left(\eta_{n}^{\mathbb{C}} \otimes_{\mathbb{C}} \operatorname{id}_{A}\right): A \rightarrow \Lambda_{n}^{\mathbb{C}} \otimes_{\mathbb{C}} A^{\prime}\right)
\end{aligned}
$$

is a bijection of sets.

Given any two objects $A$ and $A^{\prime}$ in $\mathrm{S}^{*} \mathrm{Alg}$ and any $\mathrm{SPt}^{\mathrm{op}}-$ morphism $\lambda^{\mathrm{op}}: \mathrm{pt}_{n} \rightarrow \mathrm{pt}_{m}$ (i.e. a SPt-morphism $\lambda: \mathrm{pt}_{m} \rightarrow \mathrm{pt}_{n}$ ) we can define a map of sets

$$
\operatorname{Hom}_{\mathbf{S}^{*}} \operatorname{Alg}\left(A, \Lambda_{n}^{\mathbb{C}} \otimes_{\mathbb{C}} A^{\prime}\right) \longrightarrow \operatorname{Hom}_{\mathcal{S}^{*} \operatorname{Alg}}\left(A, \Lambda_{m}^{\mathbb{C}} \otimes_{\mathbb{C}} A^{\prime}\right), \quad \varphi \longmapsto\left(\lambda^{*} \otimes_{\mathbb{C}} \operatorname{id}_{A^{\prime}}\right) \circ \varphi,
$$

where the extension of $\lambda^{*}: \Lambda_{n} \rightarrow \Lambda_{m}$ to the complexifications is implicitly understood. Using also Lemma 7.7 we obtain a map of sets

$$
\begin{aligned}
\lambda_{*}^{\mathrm{op}}: \operatorname{Hom}_{\Lambda_{n}^{\mathbb{C}}-\mathrm{S}^{*} A l g}\left(\Lambda_{n}^{\mathbb{C}} \otimes_{\mathbb{C}} A, \Lambda_{n}^{\mathbb{C}} \otimes_{\mathbb{C}} A^{\prime}\right) & \longrightarrow \operatorname{Hom}_{\Lambda_{m}^{\mathbb{C}}-\mathrm{S}^{*} \mathrm{Alg}}\left(\Lambda_{m}^{\mathbb{C}} \otimes_{\mathbb{C}} A, \Lambda_{m}^{\mathbb{C}} \otimes_{\mathbb{C}} A^{\prime}\right), \\
\kappa & \longmapsto \gamma_{\mathrm{pt}_{m}}^{-1}\left(\left(\lambda^{*} \otimes_{\mathbb{C}} \operatorname{id}_{A^{\prime}}\right) \circ \gamma_{\mathrm{pt}_{n}}(\kappa)\right) .
\end{aligned}
$$

The following properties can be easily derived from (7.17). We therefore can omit the proof.

Lemma 7.8. (i) For any identity $\mathrm{SPt}^{\mathrm{op}}$-morphism $\lambda^{\mathrm{op}}=\mathrm{id}_{\mathrm{pt}_{n}}: \mathrm{pt}_{n} \rightarrow \mathrm{pt}_{n}$ the map $\lambda_{*}^{\mathrm{op}}$ is the identity. For any two $\mathrm{SPt}^{\mathrm{op}}-$ morphisms $\lambda^{\mathrm{op}}: \mathrm{pt}_{n} \rightarrow \mathrm{pt}_{m}$ and $\lambda^{\prime \mathrm{op}}: \mathrm{pt}_{m} \rightarrow \mathrm{pt}_{l}$ we have that $\left(\lambda^{\mathrm{op}} \circ \mathrm{op} \lambda^{\mathrm{op}}\right)_{*}=\lambda_{*}^{\mathrm{op}} \circ \lambda_{*}^{\mathrm{op}}$.

(ii) $\lambda_{*}^{\text {op }}$ preserves identities and compositions, i.e.

$$
\lambda_{*}^{\mathrm{op}}\left(\operatorname{id}_{\Lambda_{n}^{\mathbb{C}} \otimes_{\mathbb{C}} A}\right)=\operatorname{id}_{\Lambda_{m}^{\mathbb{C}} \otimes_{\mathbb{C}} A}, \quad \lambda_{*}^{\mathrm{op}}\left(\kappa^{\prime} \circ \kappa\right)=\lambda_{*}^{\mathrm{op}}\left(\kappa^{\prime}\right) \circ \lambda_{*}^{\mathrm{op}}(\kappa),
$$

for all objects $A$ in $\mathrm{S}^{*} \mathrm{Alg}$ and all $\Lambda_{n}^{\mathbb{C}}$-S*Alg-morphisms $\kappa: \Lambda_{n}^{\mathbb{C}} \otimes_{\mathbb{C}} A \rightarrow \Lambda_{n}^{\mathbb{C}} \otimes_{\mathbb{C}} A^{\prime}$ and $\kappa^{\prime}: \Lambda_{n}^{\mathbb{C}} \otimes_{\mathbb{C}} A^{\prime} \rightarrow \Lambda_{n}^{\mathbb{C}} \otimes_{\mathbb{C}} A^{\prime \prime}$.

(iii) $\lambda_{*}^{\text {op }}$ preserves isomorphisms, i.e. $\kappa: \Lambda_{n}^{\mathbb{C}} \otimes_{\mathbb{C}} A \rightarrow \Lambda_{n}^{\mathbb{C}} \otimes_{\mathbb{C}} A^{\prime}$ is a $\Lambda_{n}^{\mathbb{C}}$-S*Alg-isomorphism if and only if $\lambda_{*}^{\mathrm{op}}(\kappa): \Lambda_{m}^{\mathbb{C}} \otimes_{\mathbb{C}} A \rightarrow \Lambda_{m}^{\mathbb{C}} \otimes_{\mathbb{C}} A^{\prime}$ is a $\Lambda_{m}^{\mathbb{C}}$-S*Alg-isomorphism.

With these preparations we can now define the SSet-category eS*Alg.

Definition 7.9. The SSet-category eS*Alg is given by the following data:

- The objects are all objects $A$ in $\mathrm{S}^{*} \mathrm{Alg}$. 
- For any two objects $A$ and $A^{\prime}$ in $\mathrm{eS}^{*} \mathrm{Alg}$, the object of morphisms from $A$ to $A^{\prime}$ is given by the following functor $\mathrm{eS}^{*} \operatorname{Alg}\left(A, A^{\prime}\right): \mathrm{SPt}^{\mathrm{op}} \rightarrow$ Set: For any object $\mathrm{pt}_{n}$ in $\mathrm{SPt}^{\text {op }}$ we define $\mathrm{eS}^{*} \operatorname{Alg}\left(A, A^{\prime}\right)\left(\mathrm{pt}_{n}\right)$ to be the set of all $\Lambda_{n}^{\mathbb{C}}$-S*Alg-morphisms $\kappa: \Lambda_{n}^{\mathbb{C}} \otimes_{\mathbb{C}} A \rightarrow \Lambda_{n}^{\mathbb{C}} \otimes_{\mathbb{C}} A^{\prime}$. For any $\mathrm{SPt}^{\mathrm{op}}$-morphism $\lambda^{\mathrm{op}}: \mathrm{pt}_{n} \rightarrow \mathrm{pt}_{m}$ we define the map of sets

$$
\mathrm{eS}^{*} \operatorname{Alg}\left(A, A^{\prime}\right)\left(\lambda^{\mathrm{op}}\right):=\lambda_{*}^{\mathrm{op}}: \mathrm{eS}^{*} \operatorname{Alg}\left(A, A^{\prime}\right)\left(\mathrm{pt}_{n}\right) \longrightarrow \mathrm{eS}^{*} \operatorname{Alg}\left(A, A^{\prime}\right)\left(\mathrm{pt}_{m}\right),
$$

where $\lambda_{*}^{\text {op }}$ is given in (7.17).

- For any three objects $A, A^{\prime}$ and $A^{\prime \prime}$ in eS*Alg, we define the composition morphism - : eS* $\operatorname{Alg}\left(A^{\prime}, A^{\prime \prime}\right) \times \mathrm{eS}^{*} \operatorname{Alg}\left(A, A^{\prime}\right) \rightarrow \mathrm{eS}^{*} \operatorname{Alg}\left(A, A^{\prime \prime}\right)$ to be the natural transformation with components

$$
\bullet_{\mathrm{pt}_{n}}:=0: \mathrm{eS}^{*} \operatorname{Alg}\left(A^{\prime}, A^{\prime \prime}\right)\left(\mathrm{pt}_{n}\right) \times \mathrm{eS}^{*} \operatorname{Alg}\left(A, A^{\prime}\right)\left(\mathrm{pt}_{n}\right) \longrightarrow \mathrm{eS}^{*} \operatorname{Alg}\left(A, A^{\prime \prime}\right)\left(\mathrm{pt}_{n}\right),
$$

where $\circ$ is the composition of $\Lambda_{n}^{\mathbb{C}}$-S*Alg-morphisms.

- For any object $A$ in $\mathrm{eS}^{*} \mathrm{Alg}$, we define the identity on $A$ morphism $1: \mathfrak{I} \rightarrow \mathrm{eS}^{*} \operatorname{Alg}(A, A)$ to be the natural transformation with components

$$
\mathbf{1}_{\mathrm{pt}_{n}}: \mathrm{pt}=\mathfrak{I}\left(\mathrm{pt}_{n}\right) \longrightarrow \mathrm{eS}^{*} \operatorname{Alg}(A, A)\left(\mathrm{pt}_{n}\right), \star \star \longmapsto \mathrm{id}_{\Lambda_{n}^{\mathbb{C}} \otimes_{\mathbb{C}} A}
$$

where $\operatorname{id}_{\Lambda_{n}^{\mathbb{C}} \otimes_{\mathbb{C}} A}$ is the identity $\Lambda_{n}^{\mathbb{C}}$-S*Alg-morphism.

The quantization SSet-functor $\mathfrak{e} \mathfrak{Q}: \mathrm{eX} \rightarrow \mathrm{eS}^{*} \mathrm{Alg}$ is constructed as follows: To any object $\boldsymbol{V}$ in eX we assign the object $\mathfrak{e Q}(\boldsymbol{V}):=\mathfrak{Q}(\boldsymbol{V})$ in $\mathrm{eS}^{*} \mathrm{Alg}$ that has been constructed in (5.34). To any two objects $\boldsymbol{V}$ and $\boldsymbol{V}^{\prime}$ in eX we assign the SSet-morphism $\mathfrak{e Q}_{\boldsymbol{V}, \boldsymbol{V}^{\prime}}: \operatorname{eX}\left(\boldsymbol{V}, \boldsymbol{V}^{\prime}\right) \rightarrow$ $\mathrm{eS}^{*} \operatorname{Alg}\left(\mathfrak{Q}(\boldsymbol{V}), \mathfrak{Q}\left(\boldsymbol{V}^{\prime}\right)\right)$ given by the natural transformation (of functors from SPt ${ }^{\text {op }}$ to Set) with components

$$
\left(\mathfrak{e} \mathfrak{Q}_{\boldsymbol{V}, \boldsymbol{V}^{\prime}}\right)_{\mathrm{pt}_{n}}:\left(L: \Lambda_{n} \otimes V \rightarrow \Lambda_{n} \otimes V^{\prime}\right) \mapsto\left(\left(\mathfrak{e} \mathfrak{Q}_{\boldsymbol{V}, \boldsymbol{V}^{\prime}}\right)_{\mathrm{pt}_{n}}(L): \Lambda_{n}^{\mathbb{C}} \otimes_{\mathbb{C}} \mathfrak{Q}(\boldsymbol{V}) \rightarrow \Lambda_{n}^{\mathbb{C}} \otimes_{\mathbb{C}} \mathfrak{Q}\left(\boldsymbol{V}^{\prime}\right)\right),
$$

where $\left(\mathfrak{e} \mathfrak{Q}_{\boldsymbol{V}, \boldsymbol{V}^{\prime}}\right)_{\mathrm{pt}_{n}}(L)$ is the $\Lambda_{n}^{\mathbb{C}}$-S*Alg-morphism which is specified by defining on the generators $\left(\mathfrak{e} \mathfrak{Q}_{\boldsymbol{V}, \boldsymbol{V}^{\prime}}\right)_{\mathrm{pt}_{n}}(L)\left(\zeta \otimes_{\mathbb{C}} v\right):=L(\zeta \otimes v)$, for all $v \in V$ and $\zeta \in \Lambda_{n}$. It remains to show that $\left(\mathfrak{e} \mathfrak{Q}_{\boldsymbol{V}, \boldsymbol{V}^{\prime}}\right)_{\mathrm{pt}_{n}}(L)$ is well-defined, i.e. that it preserves the two-sided super-*-ideals (5.32). Written in terms of the tensor product superalgebra $\Lambda_{n}^{\mathbb{C}} \otimes_{\mathbb{C}} \mathcal{T}_{\mathbb{C}}(\boldsymbol{V})$ the super-canonical (anti)commutation relation super-*-ideal is generated by the elements

$$
w_{1} w_{2}+(-1)^{\operatorname{dim}(S)+1}(-1)^{\left|w_{1}\right|\left|w_{2}\right|} w_{2} w_{1}-\beta \tau_{\mathrm{pt}_{n}}\left(w_{1}, w_{2}\right) \otimes_{\mathbb{C}} \mathbb{1},
$$

for all homogeneous $w_{1}, w_{2} \in \Lambda_{n} \otimes V$. Using now that by definition of eX, $\tau_{\mathrm{pt}_{n}}^{\prime} \circ\left(L \otimes_{\Lambda_{n}} L\right)=\tau_{\mathrm{pt}_{n}}$, we obtain that $\left(\mathfrak{e} \mathfrak{Q}_{\boldsymbol{V}, \boldsymbol{V}^{\prime}}\right)_{\mathrm{pt}_{n}}(L)$ is a well-defined $\Lambda_{n}^{\mathbb{C}}$-S*Alg-morphism. By direct inspection one further observes that $\mathfrak{e Q}_{\boldsymbol{V}, \boldsymbol{V}^{\prime}}$ is compatible with composition and identity. We therefore have shown

Proposition 7.10. The assignment $\mathfrak{e Q ~}: \mathrm{eX} \rightarrow \mathrm{eS}^{*} \mathrm{Alg}$ given above is a SSet-functor.

\subsection{The enriched locally covariant quantum field theory $\mathfrak{e} A$ : eSLoc $\rightarrow$ eS*Alg}

Recalling Remark A.4 we can compose the two SSet-functors $\mathfrak{e} \mathfrak{L}:$ eSLoc $\rightarrow$ eX and $\mathfrak{e} \mathfrak{Q}:$ eX $\rightarrow$ eS*Alg in order to define the SSet-functor

$$
\mathfrak{e} \mathfrak{A}:=\mathfrak{e} \mathfrak{Q} \circ \mathfrak{e} \mathfrak{L}: \mathrm{eSLoc} \longrightarrow \mathrm{eS}^{*} \mathrm{Alg} .
$$

By using the same arguments as in the proof of Theorem [5.11, the results of Theorem 7.6 imply 
Theorem 7.11. For any enriched super-field theory according to Definition 6.15 the associated $\mathrm{SSet}$-functor $\mathfrak{e A}: \mathrm{eSLoc} \rightarrow \mathrm{eS}^{*}$ Alg satisfies the following properties, for all objects $\mathrm{pt}_{n}$ in $\mathrm{SPt}^{\mathrm{op}}$ :

- Enriched locality: For any $\chi \in \operatorname{eSLoc}\left(\boldsymbol{M}, \boldsymbol{M}^{\prime}\right)\left(\mathrm{pt}_{n}\right)$, we have that $\left(\mathfrak{e A}_{\boldsymbol{M}, \boldsymbol{M}^{\prime}}\right)_{\mathrm{pt}_{n}}(\chi) \in$ $\mathrm{eS}^{*} \operatorname{Alg}\left(\mathfrak{A}(\boldsymbol{M}), \mathfrak{A}\left(\boldsymbol{M}^{\prime}\right)\right)\left(\mathrm{pt}_{n}\right)$ is monic.

- Enriched super-causality: Given $\chi_{1} \in \operatorname{eSLoc}\left(\boldsymbol{M}_{1}, \boldsymbol{M}\right)\left(\mathrm{pt}_{n}\right)$ and $\chi_{2} \in \operatorname{eSLoc}\left(\boldsymbol{M}_{2}, \boldsymbol{M}\right)\left(\mathrm{pt}_{n}\right)$,

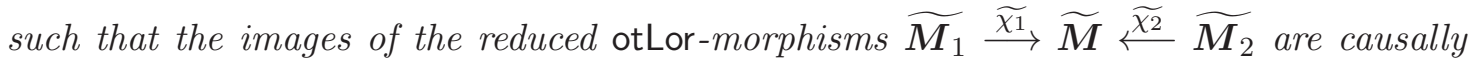
disjoint, then

$$
A_{1} A_{2}+(-1)^{\operatorname{dim}(S)+1}(-1)^{\left|A_{1}\right|\left|A_{2}\right|} A_{2} A_{1}=0,
$$

for all homogeneous $A_{1} \in\left(\mathfrak{e A}_{\boldsymbol{M}_{1}, \boldsymbol{M}}\right)_{\mathrm{pt}_{n}}\left(\chi_{1}\right)\left(\Lambda_{n}^{\mathbb{C}} \otimes_{\mathbb{C}} \mathfrak{A}\left(\boldsymbol{M}_{1}\right)\right) \subseteq \Lambda_{n}^{\mathbb{C}} \otimes_{\mathbb{C}} \mathfrak{A}(\boldsymbol{M})$ and $A_{2} \in$ $\left(\mathfrak{e} \mathfrak{A}_{\boldsymbol{M}_{2}, \boldsymbol{M}}\right)_{\mathrm{pt}_{n}}\left(\chi_{2}\right)\left(\Lambda_{n}^{\mathbb{C}} \otimes_{\mathbb{C}} \mathfrak{A}\left(\boldsymbol{M}_{2}\right)\right) \subseteq \Lambda_{n}^{\mathbb{C}} \otimes_{\mathbb{C}} \mathfrak{A}(\boldsymbol{M})$.

- Enriched time-slice axiom: Given any $\chi \in \operatorname{eSLoc}\left(\boldsymbol{M}, \boldsymbol{M}^{\prime}\right)\left(\mathrm{pt}_{n}\right)$ such that $\tilde{\chi}: \widetilde{\boldsymbol{M}} \rightarrow \widetilde{\boldsymbol{M}^{\prime}}$

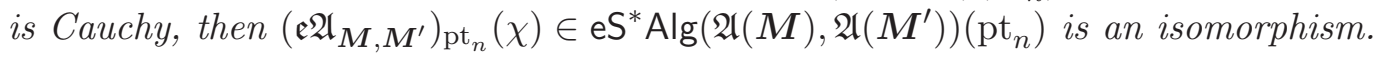

We conclude this section by showing that the SSet-functor $\mathfrak{e A}$ has an enriched locally covariant quantum field given by a SSet-natural transformation (cf. Definition A.5) $\Phi: \mathfrak{e} \mathscr{O}_{\mathrm{c}} \Rightarrow$ $\mathfrak{e A}$ of SSet-functors from eSLoc to eSVec, where $\mathfrak{e A}$ is implicitly understood to be composed with the forgetful SSet-functor eS*Alg $\rightarrow$ eSVec. We assign to every object $\boldsymbol{M}$ in eSLoc the SSetmorphism $\Phi_{\boldsymbol{M}}: \mathfrak{I} \rightarrow \operatorname{eSVec}\left(\mathscr{O}_{\mathrm{c}}(\boldsymbol{M}), \mathfrak{A}(\boldsymbol{M})\right)$ given by the natural transformation (of functors from $\mathrm{SPt}^{\mathrm{op}}$ to Set) with components

$$
\begin{aligned}
\left(\Phi_{M}\right)_{\mathrm{pt}_{n}}: \mathrm{pt}=\mathfrak{I}\left(\mathrm{pt}_{n}\right) & \longrightarrow \mathrm{eSVec}\left(\mathscr{O}_{\mathrm{c}}(\boldsymbol{M}), \mathfrak{A}(\boldsymbol{M})\right)\left(\mathrm{pt}_{n}\right), \\
\star & \longmapsto\left(\Phi_{\boldsymbol{M}}\right)_{\mathrm{pt}_{n}}(\star)=: \Phi_{\boldsymbol{M} / \mathrm{pt}_{n}}
\end{aligned}
$$

given by the $\Lambda_{n}$-SMod-morphisms

$$
\Phi_{M / \mathrm{pt}_{n}}: \Lambda_{n} \otimes \mathscr{O}_{\mathrm{c}}(\boldsymbol{M}) \longrightarrow \Lambda_{n}^{\mathbb{C}} \otimes_{\mathbb{C}} \mathfrak{A}(\boldsymbol{M}), \quad \zeta \otimes F \longmapsto \zeta \otimes_{\mathbb{C}}[F] .
$$

Notice that the diagram in Definition $\mathbf{A . 5}$ commutes, i.e.

$$
\Phi_{M^{\prime} / \mathrm{pt}_{n}} \bullet \mathrm{pt}_{n}\left(\mathfrak{e} \mathscr{O}_{\mathrm{c} \boldsymbol{M}, \boldsymbol{M}^{\prime}}\right)_{\mathrm{pt}_{n}}(\chi)=\left(\mathfrak{e A}_{M, M^{\prime}}\right)_{\mathrm{pt}_{n}}(\chi) \bullet \mathrm{pt}_{n} \Phi_{M / \mathrm{pt}_{n}},
$$

for all $\chi \in \operatorname{eSLoc}\left(\boldsymbol{M}, \boldsymbol{M}^{\prime}\right)\left(\mathrm{pt}_{n}\right)$ and all objects $\mathrm{pt}_{n}$ in SPt ${ }^{\mathrm{op}}$. In complete analogy to the ordinary case discussed in Subsection 5.5 we obtain that

$$
\Phi_{M / \mathrm{pt}_{n}}\left(P_{M / \mathrm{pt}_{n}}(H)\right)=0
$$

for all $H \in \Lambda_{n} \otimes \mathscr{O}_{\mathrm{c}}(\boldsymbol{M})$, all objects $\boldsymbol{M}$ in eSLoc and all objects $\mathrm{pt}_{n}$ in $\mathrm{SPt}^{\mathrm{op}}$, as well as the super-canonical (anti)commutation relations

$$
\Phi_{\boldsymbol{M} / \mathrm{pt}_{n}}\left(H_{1}\right) \Phi_{\boldsymbol{M} / \mathrm{pt}_{n}}\left(H_{2}\right)+(-1)^{\left|H_{1}\right|\left|H_{2}\right|} \Phi_{\boldsymbol{M} / \mathrm{pt}_{n}}\left(H_{2}\right) \Phi_{\boldsymbol{M} / \mathrm{pt}_{n}}\left(H_{1}\right)=\beta \tau_{\boldsymbol{M} / \mathrm{pt}_{n}}\left(H_{1}, H_{2}\right),
$$

for all homogeneous $H_{1}, H_{2} \in \Lambda_{n} \otimes \mathscr{O}_{\mathrm{c}}(\boldsymbol{M})$, all objects $\boldsymbol{M}$ in eSLoc and all objects pt ${ }_{n}$ in $\mathrm{SPt}^{\mathrm{op}}$.

In contrast to the ordinary case discussed in Remark 5.13, the even and odd component quantum fields do not form natural transformations in our enriched setting. The reason for this is that the push-forward $\left(\mathfrak{e} \mathscr{O}_{\mathrm{c} M, \boldsymbol{M}^{\prime}}\right)_{\mathrm{pt}_{n}}(\chi)=\chi_{*}: \Lambda_{n} \otimes \mathscr{O}_{\mathrm{c}}(\boldsymbol{M}) \rightarrow \Lambda_{n} \otimes \mathscr{O}_{\mathrm{c}}\left(\boldsymbol{M}^{\prime}\right)$ along a generic $\chi \in \operatorname{eSLoc}\left(\boldsymbol{M}, \boldsymbol{M}^{\prime}\right)\left(\mathrm{pt}_{n}\right)$ does not restrict to a $\Lambda_{n}$-SMod-morphism $\Lambda_{n} \otimes \mathscr{O}_{\mathrm{c}}^{\text {even } / \text { odd }}(\boldsymbol{M}) \rightarrow \Lambda_{n} \otimes$ 
$\mathscr{O}_{\mathrm{c}}^{\text {even/odd }}\left(\boldsymbol{M}^{\prime}\right)$. In fact, the push-forward of a homogeneous element $\mathbb{1} \otimes F \in \Lambda_{n} \otimes \mathscr{O}_{\mathrm{c}}^{\text {even/odd }}(\boldsymbol{M})$ schematically reads as

$$
\chi_{*}(\mathbb{1} \otimes F)=\sum_{I} \zeta^{I} \otimes F_{I}
$$

where $\left\{\zeta^{I} \in \Lambda_{n}\right\}$ is any adapted basis for $\Lambda_{n}$ and $F_{I} \in \mathscr{O}_{\mathrm{c}}\left(\boldsymbol{M}^{\prime}\right)$ is of $\mathbb{Z}_{2}$-parity $\left|F_{I}\right|=|F|$ $\left|\zeta_{I}\right| \bmod 2$. Hence, if there is a non-vanishing coefficient $F_{I}$ for some odd $\zeta^{I}$, then we can not restrict $\chi_{*}$ to a $\Lambda_{n}$-SMod-morphism $\Lambda_{n} \otimes \mathscr{O}_{\mathrm{c}}^{\text {even/odd }}(\boldsymbol{M}) \rightarrow \Lambda_{n} \otimes \mathscr{O}_{\mathrm{c}}^{\text {even/odd }}\left(\boldsymbol{M}^{\prime}\right)$. Those $\chi$ with this property can be identified with supersymmetry transformations, see Section 8 for some illustrating examples. In summary, our formalism implies that (as expected) supersymmetry transformations prevent the even and odd component quantum fields to be enriched natural transformations.

Remark 7.12. We would like to emphasize that the presence of the supersymmetry transformations is due to the enrichment of the involved categories over SSet. The morphism $\chi \in \operatorname{eSLoc}\left(\boldsymbol{M}, \boldsymbol{M}^{\prime}\right)\left(\mathrm{pt}_{n}\right) \subseteq \operatorname{Hom}_{\mathrm{SMan} / \mathrm{pt}_{n}}\left(M / \mathrm{pt}_{n}, M^{\prime} / \mathrm{pt}_{n}\right)$ appearing in (7.30) respects $\mathbb{Z}_{2^{-}}$ parity by definition. However, due to the presence of odd sections of the structure sheaf $\Lambda_{n}$ of $\mathrm{pt}_{n}$, the action of $\chi_{*}$ need not preserve the splittings $\mathscr{O}_{\mathrm{c}}\left(M^{(\prime)}\right)=\mathscr{O}_{\mathrm{c}}^{\text {even }}\left(M^{(\prime)}\right) \oplus \mathscr{O}_{\mathrm{c}}^{\text {odd }}\left(M^{(\prime)}\right)$ of the second tensor factor. Moreover, these odd parameters appearing in the structure sheaf $\Lambda_{n}$ of $\mathrm{pt}_{n}$ are exactly the odd quantities used in the physics literature to parametrize supersymmetry transformations.

Remark 7.13. We note that our enriched super-QFT $\mathfrak{e A}:$ eSLoc $\rightarrow$ eS*Alg cannot be restricted to its even part $\mathfrak{e A}_{0}$ (cf. Remark 5.12 for such a construction for the ordinary

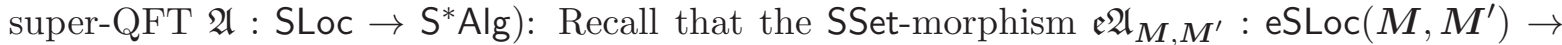
$\mathrm{eS}^{*} \operatorname{Alg}\left(\mathfrak{e} \mathfrak{A}(\boldsymbol{M}), \mathfrak{e A}\left(\boldsymbol{M}^{\prime}\right)\right.$ ) is a natural transformation (of functors from $\mathrm{SPt}^{\text {op }}$ to Set) whose components $\left(\mathfrak{e A} \mathfrak{M}_{\boldsymbol{M}, \boldsymbol{M}^{\prime}}\right)_{\mathrm{pt}_{n}}$ assign to elements $\chi \in \mathrm{eSLoc}\left(\boldsymbol{M}, \boldsymbol{M}^{\prime}\right)\left(\mathrm{pt}_{n}\right)$ certain super-*-algebra morphisms $\left(\mathfrak{e} \mathfrak{A}_{\boldsymbol{M}, \boldsymbol{M}^{\prime}}\right)_{\mathrm{pt}_{n}}(\chi): \Lambda_{n}^{\mathbb{C}} \otimes_{\mathbb{C}} \mathfrak{e} \mathfrak{A}(\boldsymbol{M}) \rightarrow \Lambda_{n}^{\mathbb{C}} \otimes_{\mathbb{C}} \mathfrak{e} \mathfrak{A}\left(\boldsymbol{M}^{\prime}\right)$. If now $\chi$ corresponds to a supersymmetry transformation, i.e. it is like in (7.30) with a non-vanishing coefficient $F_{I}$ for some odd $\zeta^{I}$, then $\left(\mathfrak{e} \mathfrak{A}_{\boldsymbol{M}, \boldsymbol{M}^{\prime}}\right)_{\mathrm{pt}_{n}}(\chi)$ does not map $\Lambda_{n}^{\mathbb{C}} \otimes_{\mathbb{C}} \mathfrak{e} \mathfrak{A}_{0}(\boldsymbol{M})$ to $\Lambda_{n}^{\mathbb{C}} \otimes_{\mathbb{C}} \mathfrak{e} \mathfrak{A}_{0}\left(\boldsymbol{M}^{\prime}\right) ;$ indeed, a supersymmetry transformation "maps an element of the even component $\mathfrak{e A}_{0}(\boldsymbol{M})$ to some element of the odd component $\mathfrak{e A}_{1}\left(\boldsymbol{M}^{\prime}\right)$ ". The fact that the even (i.e. true) observables in a supergeometric quantum field theory do not carry a representation of the supersymmetry transformations is however not problematic from the physical point of view. Supersymmetry and supergeometry are meant to serve as a selection criterion for quantum field theories by demanding that only a limited collection of observables, such as for example the action functional and quantities derived from it, transform covariantly under supersymmetry transformations.

\section{Examples}

\subsection{1|1-dimensions}

As our first example we shall study a super-field theory in 1|1-dimensions, i.e. a superparticle. For defining this theory we have to provide all the data listed in Definition 4.2 .

Representation theoretic data: We take $W=\mathbb{R}$ together with the standard 1-dimensional (Lorentz) metric

$$
g: W \otimes W \longrightarrow \mathbb{R}, \quad w_{1} \otimes w_{2} \longmapsto w_{1} w_{2} .
$$

\footnotetext{
${ }^{4}$ We would like to thank the anonymous referee for suggesting this question to us.
} 
The corresponding spin group is $\operatorname{Spin}(W, g) \simeq\{+1,-1\}$ and we take $S=\mathbb{R}$ the 1-dimensional spin representation

$$
\rho^{S}: \operatorname{Spin}(W, g) \times S \longrightarrow S,(z, s) \longmapsto z s .
$$

Notice that $\rho^{W}: \operatorname{Spin}(W, g) \times W \rightarrow W,(z, w) \mapsto w$ is the trivial representation. As $\Gamma$ we shall take the following $\operatorname{Spin}(W, g)$-equivariant symmetric pairing

$$
\Gamma: S \otimes S \longrightarrow W, s_{1} \otimes s_{2} \longmapsto s_{1} s_{2} .
$$

As positive cone we take $C:=\mathbb{R}^{+} \subset W$ and we notice that $\Gamma(s, s) \in \bar{C}$, for all $s \in S$, and $\Gamma(s, s)=0$ only for $s=0$. For $\epsilon$ we take

$$
\epsilon: S \otimes S \longrightarrow \mathbb{R}, \quad s_{1} \otimes s_{2} \longmapsto s_{1} s_{2}
$$

and we notice that it is symmetric and $\operatorname{Spin}(W, g)$-invariant. We define orientations on $W$ and $S$ by using the normalized standard bases $p=1 \in W=\mathbb{R}$ and $q=1 \in S=\mathbb{R}$. In 1|1dimensions, the super-Poincaré super-Lie algebra coincides with the supertranslation super-Lie algebra (since $\mathfrak{s p i n}$ is trivial), and we obtain for the super-Lie bracket relations in the normalized adapted basis $\{p, q\}$ for $\mathfrak{s p}=\mathfrak{t}=W \oplus S$

$$
[p, p]=0, \quad[p, q]=0, \quad[q, q]=-2 p .
$$

The objects in ghSCart: Let us characterize explicitly the objects in the category ghSCart for the case of 1|1-dimensions. To simplify our studies we shall further demand that the underlying topological spaces are connected, which is also physically reasonable as they describe a time interval. Given any such object $\boldsymbol{M}=(M, \Omega, E)$ we first notice that $\Omega=0$ since $\mathfrak{s p i n}$ is trivial in 1|1-dimensions. Moreover, the reduced 1-dimensional manifold $\widetilde{M}$ is diffeomorphic to the real line $\mathbb{R}$ as the reduced Lorentz manifold $\widetilde{\boldsymbol{M}}$ is assumed to be globally hyperbolic. The structure sheaf $\mathscr{O}_{M}$ is isomorphic to $C_{\mathbb{R}}^{\infty} \otimes \bigwedge^{\bullet} \mathbb{R}$ and the supervielbein can be expanded as

$$
E=(\gamma \mathrm{d} t+\alpha \theta \mathrm{d} \theta) \otimes p+(\delta \mathrm{d} \theta+\beta \theta \mathrm{d} t) \otimes q,
$$

where $\alpha, \beta, \gamma, \delta \in C^{\infty}(\mathbb{R})$ are coefficient functions and $t, \theta \in \mathscr{O}(\boldsymbol{M})$ are any global even/odd coordinate functions. As $E$ is by assumption non-degenerate, the functions $\gamma$ and $\delta$ have to be invertible and we may choose new coordinates (denoted with abuse of notation by the same symbols) $t \in\left(t_{0}, t_{1}\right) \subseteq \mathbb{R}$ and $\theta$, such that

$$
E=(\mathrm{d} t+\alpha \theta \mathrm{d} \theta) \otimes p+(\mathrm{d} \theta+\beta \theta \mathrm{d} t) \otimes q,
$$

where now $\alpha, \beta \in C^{\infty}\left(t_{0}, t_{1}\right)$. Coordinate functions in which $E$ takes the form (8.7) are called geometric coordinates. The supercurvature $R_{M}=0$ vanishes in 1|1-dimensions and the supertorsion is given by

$$
T_{M}=\mathrm{d} E=\left(\partial_{t} \alpha \theta \mathrm{d} t \wedge \mathrm{d} \theta+\alpha \mathrm{d} \theta \wedge \mathrm{d} \theta\right) \otimes p+(\beta \mathrm{d} \theta \wedge \mathrm{d} t) \otimes q
$$

where $\partial_{t} \alpha$ denotes the time derivative of $\alpha$. The pairing (3.11) reads as

$$
\left\langle F_{1}, F_{2}\right\rangle_{M}=\int_{t_{0}}^{t_{1}} \mathrm{~d} t\left(f_{1} h_{2}+h_{1} f_{2}\right)
$$

where we have used the expansion $F=f+\theta h \in \mathscr{O}(\boldsymbol{M})$ with $f, h \in C^{\infty}\left(t_{0}, t_{1}\right)$. 
Super-differential operators: Using the dual superderivations

$$
X=\partial_{t}-\beta \theta \partial_{\theta}, \quad D=\partial_{\theta}+\alpha \theta \partial_{t}
$$

corresponding to $E$, we define an odd super-differential operator

$$
P_{\boldsymbol{M}}:=X \circ D: \mathscr{O}(\boldsymbol{M}) \longrightarrow \mathscr{O}(\boldsymbol{M}), F=f+\theta h \longmapsto \partial_{t} h+\theta \partial_{t}\left(\alpha \partial_{t} f\right)-\theta \alpha \beta \partial_{t} f .
$$

Notice that due to the last term in (8.11), the super-differential operator $P_{M}$ is i.g. not formally super-self adjoint with respect to the pairing (8.9). If however $\beta=0$, then $P_{M}$ is formally super-self adjoint. Comparing with (8.8), the constraint $\beta=0$ can be regarded as a supertorsion constraint which demands that the odd part of the supertorsion vanishes. Such constraints also appear in supergravity, see e.g. [WZ77, Eqns. (10) and (11)].

The category SLoc: We define a full category SLoc of ghSCart by the conditions that 1.) the underlying topological spaces are connected and 2.) all supergravity supertorsion constraints given in [WZ77, Eqns. (10) and (11)] hold true, which implies that $\beta=0$ and that $\alpha$ is a constant which we fix to $\alpha=1$. Then the admissible super-Cartan supermanifolds $\boldsymbol{M}=(M, \Omega=0, E)$ are such that $\widetilde{M}=\left(t_{0}, t_{1}\right) \subseteq \mathbb{R}$ is an open interval (or $\mathbb{R}$ itself) and

$$
E=(\mathrm{d} t+\theta \mathrm{d} \theta) \otimes p+\mathrm{d} \theta \otimes q
$$

in geometric coordinates. The morphisms in SLoc can be explicitly characterized: Let $\boldsymbol{M}$ and $\boldsymbol{M}^{\prime}$ be any two objects in SLoc. A SMan-morphism $\chi: M \rightarrow M^{\prime}$ is specified by its action on the (geometric) coordinates $t^{\prime}, \theta^{\prime} \in \mathscr{O}\left(\boldsymbol{M}^{\prime}\right)$, which we can parametrize by the ansatz

$$
\chi^{*}\left(t^{\prime}\right)=a(t), \quad \chi^{*}\left(\theta^{\prime}\right)=b(t) \theta,
$$

where $a, b \in C^{\infty}\left(t_{0}, t_{1}\right)$. In order to qualify as a SLoc-morphism, $\chi$ has to preserve the supervielbeins

$$
\chi^{*}\left(E^{\prime}\right)=\left(\mathrm{d} a(t)+b(t)^{2} \theta \mathrm{d} \theta\right) \otimes p+\mathrm{d}(b(t) \theta) \otimes q=E=(\mathrm{d} t+\theta \mathrm{d} \theta) \otimes p+\mathrm{d} \theta \otimes q,
$$

which implies that $a(t)=t+c$, with $c \in \mathbb{R}$, and $b(t) \equiv 1$. Furthermore, for the reduced morphism $\widetilde{\chi}: \widetilde{M}=\left(t_{0}, t_{1}\right) \rightarrow \widetilde{M^{\prime}}=\left(t_{0}^{\prime}, t_{1}^{\prime}\right)$ to exist, the constant $c \in \mathbb{R}$ has to be such that $\left(t_{0}+c, t_{1}+c\right) \subseteq\left(t_{0}^{\prime}, t_{1}^{\prime}\right)$, i.e. $t_{0}^{\prime}-t_{0} \leq c \leq t_{1}^{\prime}-t_{1}$. For a generic $F^{\prime}=f^{\prime}\left(t^{\prime}\right)+\theta^{\prime} h^{\prime}\left(t^{\prime}\right) \in \mathscr{O}\left(\boldsymbol{M}^{\prime}\right)$, we have that

$$
\chi^{*}\left(F^{\prime}\right)=f^{\prime}(t+c)+\theta h^{\prime}(t+c) \in \mathscr{O}(\boldsymbol{M}),
$$

hence $\chi: M \rightarrow M^{\prime}$ describes a translation by $c$. It then follows automatically that such $\chi: \boldsymbol{M} \rightarrow \boldsymbol{M}^{\prime}$ are morphisms in ghSCart (and hence in SLoc), i.e. they satisfy the additional conditions imposed in Definitions 3.6 and 3.10,

The natural transformation $P: \mathscr{O} \Rightarrow \mathscr{O}$ : So far we have established the first two points in the Definition 4.2 of a super-field theory. It remains to show that (8.11) are the components of a natural transformation of formally super-self adjoint and super-Green's hyperbolic superdifferential operators. For any object $M$ in SLoc the super-differential operator (8.11) takes the form

$$
P_{M}(F)=\partial_{t} h+\theta \partial_{t}^{2} f
$$

for all $F=f+\theta h \in \mathscr{O}(\boldsymbol{M})$, from which it is clear that it is formally super-self adjoint and super-Green's hyperbolic with super-Green's operators given by

$$
G_{M}^{ \pm}(F)=G_{\partial_{t}^{2}}^{ \pm}(h)+\theta G_{\partial_{t}}^{ \pm}(f)
$$


for all $F=f+\theta h \in \mathscr{O}_{\mathrm{c}}(\boldsymbol{M})$, where $G_{\partial_{t}^{2}}^{ \pm}$and $G_{\partial_{t}}^{ \pm}$denote the retarded/advanced Green's operators for the component differential operators $\partial_{t}^{2}$ and $\partial_{t}$, respectively. The super-differential operators (8.16) are the components of a natural transformation since they are translation invariant, hence we have constructed an example of a super-field theory according to Definition 4.2. Applying Theorem 5.11 we further obtain a super-QFT, which in the 1|1-dimensional case describes a quantized superparticle.

Enriched super-field theory: We shall now show that the super-field theory defined above is also an enriched super-field theory according to Definition 6.15, Let us take any two objects $\boldsymbol{M}$ and $\boldsymbol{M}^{\prime}$ in $\mathrm{SLoc}^{\mathrm{op}}$ and any object $\mathrm{pt}_{n}$ in $\mathrm{SPt}^{\mathrm{op}}$. We characterize explicitly the set $\mathrm{eSLoc}^{\mathrm{op}}\left(\boldsymbol{M}^{\prime}, \boldsymbol{M}\right)\left(\mathrm{pt}_{n}\right)$. Any SMan/pt ${ }_{n}$-morphism $\chi: M / \mathrm{pt}_{n} \rightarrow M^{\prime} / \mathrm{pt}_{n}$ is specified by its action on the (geometric) coordinates $\mathbb{1} \otimes t^{\prime}, \mathbb{1} \otimes \theta^{\prime} \in \Lambda_{n} \otimes \mathscr{O}\left(M^{\prime}\right)$, which we can parametrize by the ansatz

$$
\begin{aligned}
& \chi^{*}\left(\mathbb{1} \otimes t^{\prime}\right)=\sum_{I \text { even }} \zeta^{I} \otimes a_{I}(t)+\sum_{I \text { odd }} \zeta^{I} \otimes b_{I}(t) \theta, \\
& \chi^{*}\left(\mathbb{1} \otimes \theta^{\prime}\right)=\sum_{I \text { even }} \zeta^{I} \otimes c_{I}(t) \theta+\sum_{I \text { odd }} \zeta^{I} \otimes d_{I}(t),
\end{aligned}
$$

where $\zeta^{I}$ is any adapted basis for the super-vector space underlying the Grassmann algebra $\Lambda_{n}$ and $a_{I}, b_{I}, c_{I}, d_{I} \in C^{\infty}\left(t_{0}, t_{1}\right)$. For $\chi \in \mathrm{eSLoc}{ }^{\mathrm{op}}\left(\boldsymbol{M}^{\prime}, \boldsymbol{M}\right)\left(\mathrm{pt}_{n}\right)$ it has to preserve the $\mathrm{pt}_{n}$-relative supervielbeins, i.e. $\chi^{*}\left(\mathbb{1} \otimes E^{\prime}\right)=\mathbb{1} \otimes E$. The odd part of this condition reads as

$$
\begin{aligned}
\chi^{*}\left(\mathbb{1} \otimes \mathrm{d} \theta^{\prime}\right) & =\left(\operatorname{id}_{\Lambda_{n}} \otimes \mathrm{d}\right)\left(\chi^{*}\left(\mathbb{1} \otimes \theta^{\prime}\right)\right) \\
& =\sum_{I \text { even }} \zeta^{I} \otimes \mathrm{d}\left(c_{I}(t) \theta\right)+\sum_{I \text { odd }} \zeta^{I} \otimes \mathrm{d}\left(d_{I}(t)\right)=\mathbb{1} \otimes \mathrm{d} \theta,
\end{aligned}
$$

and it implies that $\chi^{*}\left(\mathbb{1} \otimes \theta^{\prime}\right)=\mathbb{1} \otimes \theta+\zeta \otimes \mathbb{1}$, for some odd element $\zeta \in \Lambda_{n}$. Using this result, the even part of the above condition reads as

$$
\begin{aligned}
\chi^{*}\left(\mathbb{1} \otimes\left(\mathrm{d} t^{\prime}+\theta^{\prime} \mathrm{d} \theta^{\prime}\right)\right) & =\left(\mathrm{id}_{\Lambda_{n}} \otimes \mathrm{d}\right)\left(\chi^{*}\left(\mathbb{1} \otimes t^{\prime}\right)\right)+\chi^{*}\left(\mathbb{1} \otimes \theta^{\prime}\right)\left(\mathrm{id}_{\Lambda_{n}} \otimes \mathrm{d}\right)\left(\chi^{*}\left(\mathbb{1} \otimes \theta^{\prime}\right)\right) \\
& =\sum_{I \text { even }} \zeta^{I} \otimes \mathrm{d}\left(a_{I}(t)\right)+\sum_{I \text { odd }} \zeta^{I} \otimes \mathrm{d}\left(b_{I}(t) \theta\right)+\mathbb{1} \otimes \theta \mathrm{d} \theta+\zeta \otimes \mathrm{d} \theta \\
& =\mathbb{1} \otimes(\mathrm{d} t+\theta \mathrm{d} \theta),
\end{aligned}
$$

which implies that $\chi^{*}\left(\mathbb{1} \otimes t^{\prime}\right)=\mathbb{1} \otimes(t+c)-\zeta \otimes \theta$. As in the ordinary case, the constant $c \in \mathbb{R}$ has to satisfy $t_{0}^{\prime}-t_{0} \leq c \leq t_{1}^{\prime}-t_{1}$ for the reduced morphism $\widetilde{\chi}: \widetilde{M}=\left(t_{0}, t_{1}\right) \rightarrow \widetilde{M}^{\prime}=\left(t_{0}^{\prime}, t_{1}^{\prime}\right)$ to exist. Hence, we have shown that

$$
\operatorname{eSLoc}^{\mathrm{op}}\left(\boldsymbol{M}^{\prime}, \boldsymbol{M}\right)\left(\mathrm{pt}_{n}\right) \simeq\left\{c \in \mathbb{R}: t_{0}^{\prime}-t_{0} \leq c \leq t_{1}^{\prime}-t_{1}\right\} \times\left(\Lambda_{n}\right)_{1},
$$

where $\left(\Lambda_{n}\right)_{1}$ is the odd part of the Grassmann algebra $\Lambda_{n}=\left(\Lambda_{n}\right)_{0} \oplus\left(\Lambda_{n}\right)_{1}$. In particular, the $\mathrm{pt}_{n}$-relative automorphisms $\operatorname{eSLoc}^{\mathrm{op}}(\boldsymbol{M}, \boldsymbol{M})\left(\mathrm{pt}_{n}\right)$ are in bijective correspondence with $\mathbb{R} \times$ $\left(\Lambda_{n}\right)_{1}$ if $\widetilde{M}=\mathbb{R}$ and with $\left(\Lambda_{n}\right)_{1}$ if $\widetilde{M} \subset \mathbb{R}$ is a proper interval. These $\mathrm{pt}_{n}$-relative automorphisms describe ordinary and supertranslations. For a generic $F^{\prime}=f\left(t^{\prime}\right)+\theta^{\prime} h\left(t^{\prime}\right) \in \mathscr{O}\left(\boldsymbol{M}^{\prime}\right)$, we have that

$$
\chi^{*}\left(\mathbb{1} \otimes F^{\prime}\right)=\mathbb{1} \otimes\left(f^{\prime}(t+c)+\theta h^{\prime}(t+c)\right)+\zeta \otimes\left(h^{\prime}(t+c)-\theta \partial_{t} f^{\prime}(t+c)\right),
$$

which reproduces the usual supersymmetry transformations. The diagram (6.30) commutes, since using (8.22) one can easily compute that

$$
\chi^{*}\left(\mathbb{1} \otimes P_{M^{\prime}}\left(F^{\prime}\right)\right)=\left(\operatorname{id}_{\Lambda_{n}} \otimes P_{M}\right)\left(\chi^{*}\left(\mathbb{1} \otimes F^{\prime}\right)\right),
$$


for all $F^{\prime} \in \mathscr{O}\left(\boldsymbol{M}^{\prime}\right)$. We remind the reader that $\left(\operatorname{id}_{\Lambda_{n}} \otimes P_{\boldsymbol{M}}\right)(\zeta \otimes F)=(-1)^{|\zeta|\left|P_{\boldsymbol{M}}\right|} \zeta \otimes P_{\boldsymbol{M}}(F)$, for all homogeneous $\zeta \in \Lambda_{n}$ and $F \in \mathscr{O}(\boldsymbol{M})$. As a consequence, we have constructed an enriched super-field theory according to Definition 6.15. Applying Theorem 7.11 we further obtain an enriched super-QFT, which in the 1|1-dimensional case describes a quantized superparticle together with its supersymmetry transformations.

Supersymmetry transformations in the enriched super-QFT: In order to illustrate the structure of supersymmetry transformations let us fix any object $M$ in SLoc. The SSetfunctor $\mathfrak{e A}:$ eSLoc $\rightarrow \mathrm{eS}^{*} \mathrm{Alg}$ studied in Theorem 7.11 provides us with a superalgebra of observables $\mathfrak{A}(\boldsymbol{M})$ and a SSet-morphism $\mathfrak{e A}_{\boldsymbol{M}, \boldsymbol{M}}: \operatorname{eSLoc}(\boldsymbol{M}, \boldsymbol{M}) \rightarrow \mathrm{eS}^{*} \mathrm{Alg}(\mathfrak{A}(\boldsymbol{M}), \mathfrak{A}(\boldsymbol{M}))$ which describes the enriched automorphism group of $\mathfrak{A}(\boldsymbol{M})$. Using (8.21) and focusing only on the odd part given by $\left(\Lambda_{n}\right)_{1}$ (i.e. proper supersymmetry transformations), we obtain the $\Lambda_{n}^{\mathbb{C}}$-S*Alg-automorphisms $\left(\mathfrak{e A}_{\boldsymbol{M}, \boldsymbol{M}}\right)_{\mathrm{pt}_{n}}(\chi): \Lambda_{n}^{\mathbb{C}} \otimes_{\mathbb{C}} \mathfrak{A}(\boldsymbol{M}) \rightarrow \Lambda_{n}^{\mathbb{C}} \otimes_{\mathbb{C}} \mathfrak{A}(\boldsymbol{M})$, for any object $\mathrm{pt}_{n}$ in $\mathrm{SPt}^{\mathrm{op}}$, which can be parametrized by the odd elements $\zeta \in\left(\Lambda_{n}\right)_{1}$. Explicitly, on the generators $\Phi_{\boldsymbol{M} / \mathrm{pt}_{n}}(\mathbb{1} \otimes F)=\mathbb{1} \otimes_{\mathbb{C}}[F] \in \Lambda_{n}^{\mathbb{C}} \otimes_{\mathbb{C}} \mathfrak{A}(\boldsymbol{M})$, with $F \in \mathscr{O}_{\mathrm{c}}(\boldsymbol{M})$, these $\Lambda_{n}^{\mathbb{C}}$-S*Alg-automorphisms act as

$$
\begin{aligned}
\left(\mathfrak{e} \mathfrak{A}_{M, M}\right)_{\mathrm{pt}_{n}}(\chi)\left(\Phi_{M / \mathrm{pt}_{n}}(\mathbb{1} \otimes F)\right) & =\Phi_{\boldsymbol{M} / \mathrm{pt}_{n}}(\mathbb{1} \otimes F)-\Phi_{\boldsymbol{M} / \mathrm{pt}_{n}}(\zeta \otimes Q(F)) \\
& =\Phi_{\boldsymbol{M} / \mathrm{pt}_{n}}(\mathbb{1} \otimes F)-(\zeta \otimes \mathbb{C}) \Phi_{\boldsymbol{M} / \mathrm{pt}_{n}}(\mathbb{1} \otimes Q(F)),
\end{aligned}
$$

where $Q:=\partial_{\theta}-\theta \partial_{t}$ is the generator of supersymmetry transformations and $\zeta \in\left(\Lambda_{n}\right)_{1}$. On the superalgebra of observables $\mathfrak{A}(\boldsymbol{M})$ itself, these $\Lambda_{n}^{\mathbb{C}}$-S*Alg-automorphisms can be understood as an odd superderivation $\widehat{Q}: \mathfrak{A}(\boldsymbol{M}) \rightarrow \mathfrak{A}(\boldsymbol{M})$ : On the generators $\Phi_{\boldsymbol{M}}(F)=[F] \in \mathfrak{A}(\boldsymbol{M})$, with $F \in \mathscr{O}_{\mathrm{c}}(\boldsymbol{M})$, the superderivation $\widehat{Q}$ reads as

$$
\widehat{Q}\left(\Phi_{M}(F)\right)=-\Phi_{M}(Q(F))
$$

and it satisfies the superderivation property

$$
\widehat{Q}\left(\Phi_{M}\left(F_{1}\right) \Phi_{M}\left(F_{2}\right)\right)=\widehat{Q}\left(\Phi_{M}\left(F_{1}\right)\right) \Phi_{M}\left(F_{2}\right)+(-1)^{\left|F_{1}\right|} \Phi_{M}\left(F_{1}\right) \widehat{Q}\left(\Phi_{M}\left(F_{2}\right)\right),
$$

for all homogeneous $F_{1}, F_{2} \in \mathscr{O}_{\mathrm{c}}(\boldsymbol{M})$, as a consequence of $\left(\mathfrak{e A}_{M, M}\right)_{\mathrm{pt}_{n}}(\chi)$ being a $\Lambda_{n}^{\mathbb{C}}$-S*Algmorphism. We may decompose $\Phi_{M}$ into its component quantum fields (careful: neither $\psi_{M}$ nor $\phi_{M}$ are natural in the enriched setting) via

$$
\Phi_{\boldsymbol{M}}(F)=\psi_{\boldsymbol{M}}(f)+\phi_{\boldsymbol{M}}(h)
$$

for all $F=f+\theta h \in \mathscr{O}_{\mathbf{c}}(\boldsymbol{M})$, from which we recover the usual supersymmetry transformations

$$
\widehat{Q}\left(\psi_{\boldsymbol{M}}(f)\right)=\phi_{\boldsymbol{M}}\left(\partial_{t} f\right), \quad \widehat{Q}\left(\phi_{\boldsymbol{M}}(h)\right)=-\psi_{\boldsymbol{M}}(h),
$$

for all $f, h \in C^{\infty}\left(t_{0}, t_{1}\right)$.

\section{$8.23 \mid 2$-dimensions}

Our second example is the free Wess-Zumino model in 3|2-dimensions. There are two reasons why we prefer to analyse this toy-model instead of the physically more relevant Wess-Zumino model in 4|4-dimensions: First, in 3|2-dimensions there are only 2 odd dimensions (instead of 4 in $4 \mid 4$-dimensions), which considerably simplifies the component analysis of the superCartan structures and superfields discussed below. Second, the Wess-Zumino model in 4|4dimensions requires a chirality constraint, the implementation of which would lead to additional technical/computational complications that we would like to postpone to future work.

We provide all the data listed in Definition 4.2 , 
Representation theoretic data: We take $W=\mathbb{R}^{3}$ together with the standard Lorentz metric $g=\operatorname{diag}(1,-1,-1)$. The corresponding spin group is $\operatorname{Spin}(W, g) \simeq \operatorname{SL}(2, \mathbb{R})$ and we take $\rho^{S}: \operatorname{Spin}(W, g) \times S \rightarrow S$ the defining representation of $\operatorname{SL}(2, \mathbb{R})$ on $S=\mathbb{R}^{2}$. Notice that $\mathrm{SL}(2, \mathbb{R})$ is the two-fold cover of the identity component $\mathrm{SO}_{0}(1,2)$ of the special pseudoorthogonal group and that $\rho^{W}: \operatorname{Spin}(W, g) \times W \rightarrow W$ is given by composing the covering map with the defining representation of $\mathrm{SO}_{0}(1,2)$.

The standard basis $\left\{p_{\alpha}\right\}$ of $W=\mathbb{R}^{3}$, with $\alpha=0,1,2$, is an orthonormal basis of $(W, g)$. We denote the metric coefficients in this basis by $g_{\alpha \beta}:=g\left(p_{\alpha}, p_{\beta}\right)$ and the coefficients of the inverse metric in the dual basis $\left\{p^{\alpha}\right\}$ by $g^{\alpha \beta}$. Elements $w \in W$ will be indicated by $w=w^{\alpha} p_{\alpha}$, with summation over repeated indices understood. For the coefficients of the dual vector $g(w, \cdot)=w^{\alpha} g_{\alpha \beta} p^{\beta} \in W^{*}$ we shall also write $w_{\beta}=w^{\alpha} g_{\alpha \beta}$. Notice that $w^{\alpha}=w_{\beta} g^{\beta \alpha}$. The choice of basis $\left\{p_{\alpha}\right\}$ fixes an orientation on $W$.

The representation $\rho^{S}$ induces up to $\operatorname{SL}(2, \mathbb{R})$-equivalence a unique representation of the Clifford algebra $\mathrm{Cl}(W, g)$ in terms of purely imaginary matrices $\gamma_{\alpha}$, with $\alpha=0,1,2$, on the complexification $S_{\mathbb{C}}$ of $S$. These matrices thus satisfy the Clifford relations

$$
\gamma_{\alpha} \gamma_{\beta}+\gamma_{\beta} \gamma_{\alpha}=2 g_{\alpha \beta} \operatorname{id}_{S_{\mathbb{C}}} .
$$

Furthermore, there exists a charge conjugation matrix $C$ which satisfies

$$
\mathrm{C}^{\mathrm{T}}=-\mathrm{C}, \quad \gamma_{\alpha}^{\mathrm{T}}=-\mathrm{C} \gamma_{\alpha} \mathrm{C}^{-1},
$$

where ${ }^{\mathrm{T}}$ denotes matrix transposition. A possible representation is given in terms of the Pauli matrices by $\gamma_{0}=\sigma_{2}, \gamma_{1}=i \sigma_{1}, \gamma_{2}=i \sigma_{3}$ and $\mathbf{C}=\gamma_{0}$. We define the antisymmetrized product

$$
\gamma_{\alpha \beta}:=\frac{1}{2}\left(\gamma_{\alpha} \gamma_{\beta}-\gamma_{\beta} \gamma_{\alpha}\right)
$$

and note the identities

$$
\begin{gathered}
\left(\gamma_{\alpha} \mathrm{C}^{-1}\right)^{\mathrm{T}}=\gamma_{\alpha} \mathrm{C}^{-1}, \quad\left(\gamma_{\alpha \beta} \mathrm{C}^{-1}\right)^{\mathrm{T}} \\
=\gamma_{\alpha \beta} \mathrm{C}^{-1}, \quad \operatorname{Tr}\left(\gamma_{\alpha}\right)=\operatorname{Tr}\left(\gamma_{\alpha \beta}\right)=0, \\
\gamma_{\alpha \beta}=i \epsilon_{\alpha \beta \delta} \gamma^{\delta}, \quad L=\frac{1}{2} \operatorname{Tr}(L) \mathrm{id}_{S_{\mathbb{C}}}+\frac{1}{2} \operatorname{Tr}\left(L \gamma_{\alpha}\right) \gamma^{\alpha},
\end{gathered}
$$

where $\epsilon_{\alpha \beta \delta}$ is totally antisymmetric with $\epsilon_{012}=1$ and $L$ is an arbitrary endomorphism of $S_{\mathbb{C}}$.

As the pairing $\Gamma$ we shall take

$$
\Gamma: S \otimes S \longrightarrow W, \quad s_{1} \otimes s_{2} \longmapsto\left(s_{1}, \gamma^{\alpha} \mathrm{C}^{-1} s_{2}\right) p_{\alpha}
$$

where $(\cdot, \cdot)$ is the standard inner product on $S=\mathbb{R}^{2}$. (Notice that $\gamma^{\alpha} C^{-1}$ is a real matrix.) The pairing $\Gamma$ is symmetric, $\operatorname{Spin}(W, g)$-equivariant and positive with respect to the forward light cone $C=\left\{w \in W: g(w, w)>0\right.$ and $\left.w^{0}>0\right\}$. For $\epsilon$ we take

$$
\epsilon: S \otimes S \longrightarrow \mathbb{R}, \quad s_{1} \otimes s_{2} \longmapsto\left(s_{1}, i \mathrm{C}^{-1} s_{2}\right),
$$

which is $\operatorname{Spin}(W, g)$-invariant, antisymmetric and non-degenerate. We choose a symplectic basis $\left\{q_{a}\right\}$ of $S=\mathbb{R}^{2}$, with $a=1,2$, and use the index notation $\epsilon_{a b}:=\epsilon\left(q_{a}, q_{b}\right)$ with $\epsilon_{12}=-\epsilon_{21}=1$. We further set $\epsilon^{a b}:=\epsilon_{a b}$ for the coefficients of the symplectic structure on the dual vector space $S^{*}$ in the dual basis $\left\{q^{a}\right\}$ and notice that $\epsilon^{a b} \epsilon_{b c}=-\delta_{c}^{a}$. Elements $s \in S$ will be indicated by $s=s^{a} q_{a}$, with summation over repeated indices understood. For the coefficients of the dual spinor $\epsilon(s, \cdot)=s^{a} \epsilon_{a b} q^{b} \in S^{*}$ we shall also write $s_{b}=s^{a} \epsilon_{a b}$. Notice that $s^{a}=s_{b} \epsilon^{a b}$. The choice of basis $\left\{q_{a}\right\}$ fixes an orientation on $S$. The two pairings $\Gamma$ and $\epsilon \operatorname{read}$ in our bases as

$$
\Gamma\left(s_{1}, s_{2}\right)=-s_{1}^{a} s_{2}^{b} i \gamma_{a b}^{\alpha} p_{\alpha}, \quad \epsilon\left(s_{1}, s_{2}\right)=s_{1}^{a} s_{2}^{b} \epsilon_{a b} .
$$


In order to state explicitly the super-Lie bracket relations in the super-Poincaré super-Lie algebra $\mathfrak{s p}=(\mathfrak{s p i n} \oplus W) \oplus S$ corresponding to this choice of data, we recall that the Lie algebra $\mathfrak{s p i n}$ may be spanned by generators $L_{\alpha \beta}=-L_{\beta \alpha}$, with $\alpha, \beta=0,1,2$, and that the Lie algebra actions induced by $\rho^{W}$ and $\rho^{S}$ read as

$$
\begin{aligned}
\rho_{*}^{W}: \mathfrak{s p i n} \otimes W & \longrightarrow W, \quad L_{\alpha \beta} \otimes p_{\gamma} \longmapsto g_{\alpha \gamma} p_{\beta}-g_{\beta \gamma} p_{\alpha}, \\
\rho_{*}^{S}: \mathfrak{s p i n} \otimes S \longrightarrow S, \quad L_{\alpha \beta} \otimes q_{a} & \longmapsto \frac{1}{2} \gamma_{\alpha \beta_{a}}{ }^{b} q_{b} .
\end{aligned}
$$

Thus, the super-Lie bracket relations in $\mathfrak{s p}$ read in the adapted basis $\left\{p_{\alpha}, q_{a}\right\}$ as

$$
\begin{gathered}
{\left[L_{\alpha \beta}, L_{\gamma \delta}\right]=g_{\beta \gamma} L_{\alpha \delta}+g_{\alpha \delta} L_{\beta \gamma}-g_{\alpha \gamma} L_{\beta \delta}-g_{\beta \delta} L_{\alpha \gamma},} \\
{\left[L_{\alpha \beta}, p_{\gamma}\right]=g_{\alpha \gamma} p_{\beta}-g_{\beta \gamma} p_{\alpha}, \quad\left[L_{\alpha \beta}, q_{a}\right]=\frac{1}{2} \gamma_{\alpha \beta}{ }_{a}{ }^{b} q_{b},} \\
{\left[p_{\alpha}, p_{\beta}\right]=\left[p_{\alpha}, q_{a}\right]=0, \quad\left[q_{a}, q_{b}\right]=2 i \gamma_{a b}^{\alpha} p_{\alpha} .}
\end{gathered}
$$

The objects in ghSCart: We characterize explicitly the objects $\boldsymbol{M}=(M, \Omega, E)$ in the category ghSCart for the case of 3|2-dimensions under the following simplifying assumptions: As in the 1|1-dimensional case, we assume that the underlying topological spaces $\widetilde{M}$ are connected and furthermore that the structure sheaf $\mathscr{O}_{M}$ is globally isomorphic to $C_{\widetilde{M}}^{\infty} \otimes \bigwedge^{\bullet} \mathbb{R}^{2}$. Due to the latter assumption, there exist global odd coordinate functions $\theta^{a}$, with $a=1,2$, and we set (with abuse of notation)

$$
\theta^{2}:=-\epsilon_{a b} \theta^{a} \theta^{b}=-2 \theta^{1} \theta^{2}
$$

Notice that

$$
\theta^{a} \theta^{b}=-\frac{1}{2} \epsilon^{a b} \theta^{2}
$$

The most general even supervielbein $E=e^{\alpha} \otimes p_{\alpha}+\xi^{a} \otimes q_{a} \in \Omega^{1}(M, \mathfrak{t}$ ) (summation over repeated indices understood) is given by

$$
\begin{aligned}
e^{\alpha} & =\widetilde{e}^{\beta}\left(\delta_{\beta}{ }^{\alpha}+j_{\beta}{ }^{\alpha} \frac{\theta^{2}}{2}\right)-\mathrm{d} \theta^{b} h_{b c}^{\alpha} \theta^{c}, \\
\xi^{a} & =\widetilde{e}^{\beta} l_{\beta b}{ }^{a} \theta^{b}+\mathrm{d} \theta^{b}\left(\delta_{b}{ }^{a}+k_{b}{ }^{a} \frac{\theta^{2}}{2}\right),
\end{aligned}
$$

where $\widetilde{E}=\widetilde{e}^{\alpha} \otimes p_{\alpha}$ is the vielbein on the reduced Lorentz manifold $\widetilde{M}$ and $j_{\beta}{ }^{\alpha}, h_{b c}^{\alpha}, l_{\beta b}{ }^{a}, k_{b}{ }^{a} \in$ $C^{\infty}(\widetilde{M})$ are coefficient functions. Notice that we have chosen the odd coordinates $\theta^{b}$ in such a way that the coefficient of $\mathrm{d} \theta^{b}$ in $\xi^{a}$ has a very simple form. This is always possible due to the assumption of non-degeneracy of $E$. Similar to the 1|1-dimensional case, we call such odd coordinates geometric coordinates. We introduce the dual Lorentz vielbein $\widetilde{e}_{\alpha}$ by the duality condition $\left\langle\widetilde{e}_{\alpha}, \widetilde{e}^{\beta}\right\rangle=\delta_{\alpha}{ }^{\beta}$ and the dual superderivations $\partial_{a}$ by $\left\langle\partial_{a}, \mathrm{~d} \theta^{b}\right\rangle=\delta_{a}{ }^{b}$. Notice that $\left\langle\partial_{a}, \widetilde{e}^{\alpha}\right\rangle=\left\langle\widetilde{e}_{\alpha}, \mathrm{d} \theta^{a}\right\rangle=0$. Using these dual superderivations, we can write the inverse supervielbein as

$$
\begin{aligned}
& e_{\alpha}=\left(\delta_{\alpha}{ }^{\beta}+J_{\alpha}{ }^{\beta} \frac{\theta^{2}}{2}\right) \widetilde{e}_{\beta}-l_{\alpha c}{ }^{b} \theta^{c} \partial_{b}, \quad \text { with } J_{\alpha}{ }^{\beta}:=-j_{\alpha}{ }^{\beta}+l_{\alpha c}{ }^{b} h_{b}^{\beta c}, \\
& \xi_{a}=h_{a b}^{\beta} \theta^{b} \widetilde{e}_{\beta}+\left(\delta_{a}{ }^{b}+K_{a}{ }^{b} \frac{\theta^{2}}{2}\right) \partial_{b}, \quad \text { with } K_{a}{ }^{b}:=-k_{a}{ }^{b}-h_{a}^{\beta c} l_{\beta c}{ }^{b} .
\end{aligned}
$$


The duality relations $\left\langle e_{\alpha}, e^{\beta}\right\rangle=\delta_{\alpha}{ }^{\beta},\left\langle\xi_{a}, \xi^{b}\right\rangle=\delta_{a}{ }^{b}$ and $\left\langle\xi_{a}, e^{\alpha}\right\rangle=\left\langle e_{\alpha}, \xi^{a}\right\rangle=0$ hold true. For the super-spin connection, the most general even $\Omega \in \Omega^{1}(M, \mathfrak{s p i n})$ can be expanded as

$$
\Omega=\widetilde{e}^{\alpha}\left(\omega_{\alpha}+\lambda_{\alpha} \frac{\theta^{2}}{2}\right)+\mathrm{d} \theta^{a} \phi_{a b} \theta^{b},
$$

where $\omega_{\alpha}, \lambda_{\alpha}, \phi_{a b} \in C^{\infty}(\widetilde{M}, \mathfrak{s p i n})$ are coefficient functions with values in the Lie algebra $\mathfrak{s p i n}$.

The category SLoc: We define a full subcategory SLoc of ghSCart by the conditions that 1.) the underlying topological spaces are connected, 2.) the structure sheaves are globally isomorphic to $C_{\widetilde{M}}^{\infty} \otimes \bigwedge^{\bullet} \mathbb{R}^{2}$ and 3.) the supergravity supertorsion constraints given in WZ77, Eqns. (10) and (11)] hold true. In order to discuss the latter constraints, we consider an arbitrary (local) coordinate system $x^{\mu}$ on $\widetilde{M}$, with $\mu=0,1,2$, and use the notation $X^{M}$ for the combined super-coordinate system $\left\{x^{\mu}, \theta^{m}\right\}$, where $\theta^{m}$ are global geometric odd coordinates. (With abuse of notation we will denote the indices on $X^{M}$ by the same symbol as the supermanifold.) We set $|M|:=\left|X^{M}\right|$ for the $\mathbb{Z}_{2}$-parity of $X^{M}$. Analogously, we use the notation $P_{A}$ for the combined generators $\left\{p_{\alpha}, q_{a}\right\}$ of the supertranslation super-Lie algebra $\mathfrak{t}=W \oplus S$ and set $|A|:=\left|P_{A}\right|$. Consequently, we can expand the supervielbein as $E=E^{A} \otimes P_{A}=\mathrm{d} X^{M} E_{M}^{A} \otimes P_{A}$ and its inverse as $E_{A}=E_{A}^{M} \partial_{M}$. Using this notation, we may expand and compute the supertorsion (3.7)

$$
T_{M}=T^{A} \otimes P_{A}=\frac{1}{2} E^{B} \wedge E^{C} T_{B C}{ }^{A} \otimes P_{A},
$$

where

$$
T_{B C}{ }^{A}=(-1)^{|M||C|} E_{B}^{M} E_{C}^{N}\left(\partial_{N} E_{M}^{A}-(-1)^{|N||M|} \partial_{M} E_{N}^{A}\right)+\Omega_{B C}{ }^{A}-(-1)^{|B||C|} \Omega_{C B}{ }^{A},
$$

and

$$
\Omega_{C B}{ }^{A}:= \begin{cases}\left\langle E_{C}, \Omega_{B}{ }^{A}\right\rangle & , \text { if }(A, B)=(\alpha, \beta) \text { or }(A, B)=(a, b) \\ 0 & , \text { otherwise }\end{cases}
$$

Here, $\Omega_{\alpha}{ }^{\beta}$ and $\Omega_{a}{ }^{b}$ are defined, for arbitrary $w=w^{\alpha} p_{\alpha} \in W$ and $s=s^{a} q_{a} \in S$, as

$$
\rho_{*}^{W}(\Omega \otimes w)=: w^{\alpha} \Omega_{\alpha}{ }^{\beta} \otimes p_{\beta}, \quad \rho_{*}^{S}(\Omega \otimes s)=: s^{a} \Omega_{a}^{b} \otimes q_{b} .
$$

Note that the signs in (8.42b) are only correct if $E$ is even.

The supertorsion constraints introduced in [WZ77] read as

$$
T_{b c}{ }^{\alpha}=2 i \gamma_{b c}^{\alpha}, \quad T_{\beta c}{ }^{a}=T_{b c}{ }^{a}=T_{\beta \gamma}{ }^{\alpha}=T_{\beta c}{ }^{\alpha}=0 .
$$

It is remarkable that these constraints, together with the requirement that $E$ and $\Omega$ are even, determine the supervielbein and super-spin connection uniquely in terms of the reduced Lorentz vielbein $\widetilde{E}$. In particular, demanding that $E$ is even rules out a non-vanishing Rarita-Schwinger field (gravitino) in the expansion of $E$. The unique solution to the supertorsion constraints is

$$
\begin{gathered}
j_{\alpha}{ }^{\beta}=-i \gamma^{\beta}{ }_{a}^{b}\left(\omega_{\alpha}\right)_{b}{ }^{a}=\frac{1}{2} \epsilon^{\beta \gamma \delta}\left(\omega_{\alpha}\right)_{\gamma \delta}, \quad J_{\alpha}{ }^{\beta}=0 \\
h_{a b}^{\alpha}=i \gamma_{a b}^{\alpha}, \quad l_{\alpha a}{ }^{b}=-\left(\omega_{\alpha}\right)_{a}{ }^{b}, \quad k_{a}{ }^{b}=0, \quad K_{a}{ }^{b}=i \gamma^{\beta}{ }_{a}{ }^{c}\left(\omega_{\beta}\right)_{c}{ }^{b} \\
\left(\omega_{\beta}\right)_{\gamma}{ }^{\alpha}-\left(\omega_{\gamma}\right)_{\beta}{ }^{\alpha}=\widetilde{e}_{\beta}^{\mu} \widetilde{e}_{\gamma}^{\nu}\left(\partial_{\mu} \widetilde{e}_{\nu}^{\alpha}-\partial_{\nu} \widetilde{e}_{\mu}^{\alpha}\right), \quad \phi_{a b}=0, \quad\left(\lambda_{\alpha}\right)_{\beta}{ }^{\gamma}=-\operatorname{Ric}_{\alpha \delta} \epsilon_{\beta}{ }^{\gamma \delta}
\end{gathered}
$$


where the first identity in (8.45c) implies that $\omega=\widetilde{e}^{\alpha} \omega_{\alpha}$ is the connection one-form of the Levi-Civita connection and Ric denotes the corresponding Ricci curvature tensor, cf. Wal84, Section 3.4].

The Berezinian density $\operatorname{Ber}(E)$ has a particularly simple form for objects $\boldsymbol{M}=(M, \Omega, E)$ in SLoc. In fact, for a general

$$
F=\varphi+\psi_{a} \theta^{a}+\eta \frac{\theta^{2}}{2} \in \mathscr{O}_{\mathrm{c}}(\boldsymbol{M})
$$

one has

$$
\int_{M} \operatorname{Ber}(E) F=\int_{\widetilde{M}} \operatorname{vol}_{\widetilde{M}}\left(\eta+\varphi\left(j_{\alpha}{ }^{\alpha}-l_{\alpha c}{ }^{b} h_{b}^{\alpha c}-k_{a}{ }^{a}\right)\right)=\int_{\widetilde{M}} \operatorname{vol}_{\widetilde{M}} \eta,
$$

where $\operatorname{vol}_{\widetilde{M}}$ is the canonical volume form on the reduced oriented Lorentz manifold. Consequently, the pairing (3.11) reads as

$$
\left\langle F_{1}, F_{2}\right\rangle_{M}=\int_{\widetilde{M}} \operatorname{vol}_{\widetilde{M}}\left(\varphi_{1} \eta_{2}+\varphi_{2} \eta_{1}+\psi_{1}^{a} \psi_{2 a}\right),
$$

where the expansion (8.46) has been used.

The natural transformation $P: \mathscr{O} \Rightarrow \mathscr{O}$ : Given any super-Cartan supermanifold $M$, we can consider the super-differential operator $\mathscr{D}_{\Omega}^{a} \circ \mathscr{D}_{a}: \mathscr{O}(\boldsymbol{M}) \rightarrow \mathscr{O}(\boldsymbol{M})$ defined by

$$
\begin{aligned}
\mathscr{D}_{a}(F) & :=\xi_{a}(F), \\
\mathscr{D}_{\Omega b} \circ \mathscr{D}_{a}(F) & :=\xi_{b}\left(\mathscr{D}_{a}(F)\right)+\left\langle\xi_{b}, \Omega_{a}{ }^{c}\right\rangle \mathscr{D}_{c}(F), \\
\mathscr{D}_{\Omega}^{a} \circ \mathscr{D}_{a}(F) & :=\epsilon^{a b} \mathscr{D}_{\Omega b} \circ \mathscr{D}_{a}(F),
\end{aligned}
$$

for all $F \in \mathscr{O}(\boldsymbol{M})$. For any object $\boldsymbol{M}$ in SLoc, one may compute using the expansion (8.46)

$$
\begin{aligned}
\mathscr{D}_{\Omega}^{a} \circ \mathscr{D}_{a}(F)= & -2 \eta \\
& +\left(2 h^{\alpha}{ }_{a}{ }^{b} \widetilde{e}_{\alpha}\left(\psi_{b}\right)+K_{a}{ }^{b} \psi_{b}+h^{\alpha}{ }_{a}{ }^{b}\left(\omega_{\alpha}\right)_{b}{ }^{c} \psi_{c}+\left(\phi_{a}{ }^{b}\right)_{b}{ }^{c} \psi_{c}\right) \theta^{a} \\
& -\left(2 h^{\alpha}{ }_{a}{ }^{b} \widetilde{e}_{\alpha}\left(h^{\beta}{ }_{b}{ }^{a} \widetilde{e}_{\beta}(\varphi)\right)+\left({K_{d}}^{c}+h^{\beta}{ }_{d}{ }^{a}\left(\omega_{\beta}\right)_{a}{ }^{c}+\left(\phi_{d}{ }^{a}\right)_{a}{ }^{c}\right) h^{\alpha}{ }_{c}{ }^{d} \widetilde{e}_{\alpha}(\varphi)\right) \frac{\theta^{2}}{2} \\
& +\left(h^{\beta}{ }_{a}^{b}\left(\omega_{\beta}\right)_{b}{ }^{a}+\left(\phi_{a}{ }^{b}\right)_{b}{ }^{a}\right) \eta \frac{\theta^{2}}{2} \\
= & -2 \eta+2(i \not \nabla \psi)_{a} \theta^{a}+2 \square \varphi \frac{\theta^{2}}{2}
\end{aligned}
$$

where $i \not \nabla$ is the geometric Dirac operator and $\square$ is the geometric d'Alembert operator. Here we used the essential identity

$$
\sigma:=\gamma_{a}^{\beta}{ }_{a}^{b}\left(\omega_{\beta}\right)_{b}{ }^{a}=\frac{i}{2}\left(\omega_{\alpha}\right)_{\beta \gamma} \epsilon^{\alpha \beta \gamma}=0,
$$

which holds because for each point $x \in \widetilde{M}$ one can find a vielbein $\widetilde{E}$ such that $\left(\omega_{\alpha}\right)_{\beta \gamma}(x)=0$ and thus $\sigma(x)=0$. However, $\sigma$ is a scalar invariant and thus $\sigma \equiv 0$ independent of the chosen vielbein.

For any object $M$ in SLoc and any constant $m \geq 0$, we define the super-differential operator

$$
P_{M}:=\frac{1}{2} \mathscr{D}_{\Omega}^{a} \circ \mathscr{D}_{a}+m \operatorname{id}_{\mathscr{O}(\boldsymbol{M})}: \mathscr{O}(\boldsymbol{M}) \longrightarrow \mathscr{O}(\boldsymbol{M})
$$


Solutions of $P_{M}(F)=0$ satisfy

$$
-\eta+m \varphi=0, \quad i \not \nabla \psi_{a}+m \psi_{a}=0, \quad \square \varphi+m \eta=0,
$$

and thus in particular $\psi_{a}$ is a solution of the Dirac equation and $\varphi$ is a solution of the KleinGordon equation $\square \varphi+m^{2} \varphi=0$. The super-differential operator $P_{M}$ is formally super-self adjoint with respect to the pairing (8.48). In general, one may show for arbitrary homogeneous $F_{1} \in \mathscr{O}(\boldsymbol{M})$ and arbitrary $F_{2} \in \mathscr{O}_{\mathrm{c}}(\boldsymbol{M})$ that (see e.g. [BK98, Section 5.2.8.])

$$
\int_{M} \operatorname{Ber}(E) F_{1} \mathscr{D}_{\Omega}^{a} \circ \mathscr{D}_{a}\left(F_{2}\right)=(-1)^{1+\left|F_{1}\right|} \int_{M} \operatorname{Ber}(E) \mathscr{D}^{a}\left(F_{1}\right) \mathscr{D}_{a}\left(F_{2}\right)
$$

if $T_{a \beta}{ }^{\beta}-T_{a b}{ }^{b}=0$ and thus not all supertorsion constraints are necessary for the formal superself adjointness of $P_{M}$. Notice that $P_{M}$ is also super-Green's hyperbolic: If we write $P_{M}$ in matrix form as

$$
P_{M}=\left(\begin{array}{ccc}
m & 0 & -1 \\
0 & i \not \nabla+m & 0 \\
\square & 0 & m
\end{array}\right),
$$

then the retarded/advanced super-Green's operators $G_{\boldsymbol{M}}^{ \pm}$for $P_{\boldsymbol{M}}$ can be written as

$$
G_{M}^{ \pm}=\left(\begin{array}{ccc}
m G_{\square+m^{2}}^{ \pm} & 0 & G_{\square+m^{2}}^{ \pm} \\
0 & G_{i \not \nabla+m}^{ \pm} & 0 \\
-\square \circ G_{\square+m^{2}}^{ \pm} & 0 & m G_{\square+m^{2}}^{ \pm}
\end{array}\right),
$$

where $G_{\square+m^{2}}^{ \pm}$and $G_{i \not \nabla+m}^{ \pm}$are the retarded/advanced Green's operators for the component differential operators $\square+m^{2}$ and $i \not \nabla+m$, respectively. Finally, the super-differential operators (8.52) are the components of a natural transformation since they are constructed geometrically in terms of the supervielbein $E$ and a constant $m \geq 0$. Hence, we have constructed an example of a super-field theory according to Definition 4.2. Applying Theorem 5.11 we further obtain a super-QFT, which in the present case describes the quantized free Wess-Zumino model in $3 \mid 2$-dimensions.

Enriched super-field theory: We shall now discuss the super-field theory defined above in the enriched setting. We consider two objects $\boldsymbol{M}$ and $\boldsymbol{M}^{\prime}$ in eSLoc ${ }^{\mathrm{op}}$ and any object $\mathrm{pt}_{n}$ in $\mathrm{SPt}^{\mathrm{op}}$. Before discussing the set $\operatorname{eSLoc}^{\mathrm{op}}\left(\boldsymbol{M}^{\prime}, \boldsymbol{M}\right)\left(\mathrm{pt}_{n}\right)$ in more detail, we remark that, since any $\chi \in \mathrm{eSLoc}^{\mathrm{op}}\left(\boldsymbol{M}^{\prime}, \boldsymbol{M}\right)\left(\mathrm{pt}_{n}\right)$ preserves by definition the $\mathrm{pt}_{n}$-relative supervielbeins, and $P_{\boldsymbol{M}}$ is constructed geometrically, the super-field theory discussed in this example automatically satisfies the axioms of an enriched super-field theory given in Definition 6.15.

Instead of fully characterizing the set $\operatorname{eSLoc}{ }^{\mathrm{op}}\left(\boldsymbol{M}^{\prime}, \boldsymbol{M}\right)\left(\mathrm{pt}_{n}\right)$, we aim for analyzing a presumably large subset which contains supersymmetry transformations by considering a wellmotivated ansatz. For a generic $F^{\prime} \in \mathscr{O}_{\mathrm{c}}\left(\boldsymbol{M}^{\prime}\right)$ expanded as in (8.46), we consider SMan $/ \mathrm{pt}_{n^{-}}$ morphisms $\chi: M / \mathrm{pt}_{n} \rightarrow M^{\prime} / \mathrm{pt}_{n}$ of the form

$$
\begin{aligned}
& \chi^{*}\left(\mathbb{1} \otimes F^{\prime}\right)=\mathbb{1} \otimes F+\zeta \otimes Q(F), \\
& F:=\tilde{\chi}^{*}\left(\varphi^{\prime}\right)+\tilde{\chi}^{*}\left(\psi_{a}^{\prime}\right) \theta^{a}+\tilde{\chi}^{*}\left(\eta^{\prime}\right) \frac{\theta^{2}}{2},
\end{aligned}
$$

where $\zeta \in \Lambda_{n}$ is odd, $\tilde{\chi}: \widetilde{M} \rightarrow \widetilde{M^{\prime}}$ is a smooth map which preserves the reduced Lorentz vielbein $\widetilde{\chi}^{*}\left(\widetilde{E^{\prime}}\right)=\widetilde{E}$ and $Q$ is an odd superderivation. A necessary condition for such $\chi$ to be an element of $\mathrm{eSLoc}^{\mathrm{op}}\left(\boldsymbol{M}^{\prime}, \boldsymbol{M}\right)\left(\mathrm{pt}_{n}\right)$ is that $Q$ supercommutes with the dual supervielbein, i.e.

$$
\left[Q, \xi_{a}\right]=0, \quad\left[Q, e_{\alpha}\right]=0 .
$$


We can expand $Q$ as

$$
Q=A_{b}^{\alpha} \theta^{b} \widetilde{e}_{\alpha}+\left(B^{a}+C^{a} \frac{\theta^{2}}{2}\right) \partial_{a}
$$

and evaluate the constraints (8.58) in the given order. After a straightforward computation we find that these constraints are satisfied if and only if

$$
B^{c}\left(\omega_{\alpha}\right)_{c}^{a}=0, \quad \widetilde{e}_{\alpha}\left(B^{c}\right)=0, \quad A_{b}^{\alpha}=-i B^{c} \gamma_{b c}^{\alpha}, \quad C^{b}=0 .
$$

Thus, we find that a non-zero $Q$ is only possible if the Levi-Civita connection $\omega$ is vanishing, and hence also the super-spin connection $\Omega$ is vanishing on $\boldsymbol{M}$. In this case $\chi$ defined as above is an element of $\operatorname{eSLoc}^{\mathrm{op}}\left(\boldsymbol{M}^{\prime}, \boldsymbol{M}\right)\left(\mathrm{pt}_{n}\right)$ if and only if the super-spin connection $\Omega^{\prime}$ on $\boldsymbol{M}^{\prime}$ is also vanishing.

This rather restrictive condition for the existence of interesting enriched morphisms $\chi \in$ $\mathrm{eSLoc}^{\mathrm{op}}\left(\boldsymbol{M}^{\prime}, \boldsymbol{M}\right)\left(\mathrm{pt}_{n}\right)$ originates from our requirement that the supervielbein $E$ is even and that $\chi$ must preserve the $\mathrm{pt}_{n}$-relative supervielbein. In the treatment of supergravity one usually deals with supervielbeins which are not purely even and considers, in the terminology of this paper, enriched morphisms which have to preserve the $\mathrm{pt}_{n}$-relative supervielbein and $\mathrm{pt}_{n}$-relative super-spin connection only up to local Lorentz transformations. This class of enriched morphisms contains the so-called supergravity transformations [WB92].

Supersymmetry transformations in the enriched super-QFT: We close the discussion of this example in analogy to the 1|1-dimensional case by illustrating the structure of supersymmetry transformations. As discussed above, these transformations appear only if we consider an object $\boldsymbol{M}=(M, \Omega, E)$ in eSLoc with $\Omega=0$, such as for example the $3 \mid 2$-dimensional superMinkowski space. Given any such object, we can use the SSet-functor $\mathfrak{e} A$ : eSLoc $\rightarrow$ eS*Alg constructed in Theorem 7.11 to obtain a superalgebra of observables $\mathfrak{A}(\boldsymbol{M})$ and a SSet-morphism $\mathfrak{e A}_{\boldsymbol{M}, \boldsymbol{M}}: \operatorname{eSLoc}(\boldsymbol{M}, \boldsymbol{M}) \rightarrow \mathrm{eS}^{*} \operatorname{Alg}(\mathfrak{A}(\boldsymbol{M}), \mathfrak{A}(\boldsymbol{M}))$, which describes the enriched automorphism group of $\mathfrak{A}(\boldsymbol{M})$. Proper supersymmetry transformations are described by the odd superderivations

$$
Q_{B}:=B^{a} \partial_{a}-i B^{a} \gamma_{a b}^{\alpha} \theta^{b} \widetilde{e}_{\alpha}
$$

with $B^{a}$ a constant spinor, and they are parametrized by odd elements $\zeta \in\left(\Lambda_{n}\right)_{1}$. As in the 1|1-dimensional case, such supersymmetry transformations may be understood as odd superderivations $\widehat{Q}_{B}: \mathfrak{A}(\boldsymbol{M}) \rightarrow \mathfrak{A}(\boldsymbol{M})$ which act on the generators $\Phi_{\boldsymbol{M}}(F)=[F] \in \mathfrak{A}(\boldsymbol{M})$, with $F \in \mathscr{O}_{\mathrm{c}}(\boldsymbol{M})$, as

$$
\widehat{Q_{B}}\left(\Phi_{M}(F)\right)=-\Phi_{M}\left(Q_{B}(F)\right) .
$$

We may decompose $\Phi_{M}(F)$ into its component quantum fields by using the expansion

$$
F=f+\rho_{a} \theta^{a}+h \frac{\theta^{2}}{2} .
$$

Explicitly, we set

$$
\Phi_{M}(F)=\phi_{\boldsymbol{M}}(f)+\psi_{\boldsymbol{M}}^{a}\left(\rho_{a}\right)+\eta_{\boldsymbol{M}}(h),
$$

and we recover the usual supersymmetry transformations

$$
\begin{aligned}
& \widehat{Q_{B}}\left(\phi_{\boldsymbol{M}}(f)\right)=\psi_{\boldsymbol{M}}^{a}\left(f B_{a}\right), \\
& \widehat{Q^{\prime}}\left(\psi_{M}^{a}\left(\rho_{a}\right)\right)=\phi_{\boldsymbol{M}}\left(B^{a} i \not \nabla \rho_{a}\right)+\eta_{\boldsymbol{M}}\left(B^{a} \rho_{a}\right), \\
& \widehat{Q_{B}}\left(\eta_{\boldsymbol{M}}(h)\right)=-\psi_{\boldsymbol{M}}^{a}\left(i \not \nabla\left(h B_{a}\right)\right) .
\end{aligned}
$$




\section{Acknowledgements}

We would like to thank the referees for valuable comments and suggestions. We thank Chris Fewster for useful comments on this work. A.S. also thanks Sven Meinhardt for many general discussions on supergeometry. The work of T.-P.H. and A.S. is supported by a Research Fellowship of Deutsche Forschungsgemeinschaft (DFG) and F.H. is supported by the SFB 647:Raum-Zeit-Materie funded by the DFG. Furthermore, T.-P.H. and A.S. would like to thank Mathematisches Forschungsinstitut Oberwolfach (MFO) for the support under the program Research in Pairs and the great hospitality at the institute.

\section{A Basics of enriched category theory}

We review some elementary definitions of enriched category theory which will be used in our work. For detailed introductions to this subject see e.g. [Kel82] and [BS00].

Let $\mathrm{V}$ be a monoidal category. For our purposes we can assume that the associator in $\mathrm{V}$ is trivial. We denote the monoidal bifunctor by $\otimes: \mathrm{V} \times \mathrm{V} \rightarrow \mathrm{V}$ and the unit object in $\mathrm{V}$ by $I$.

Definition A.1. A V-category (or a category enriched over V) C consists of

- a class $\mathrm{Ob}(\mathrm{C})$ of objects;

- for all objects $A, B \in \mathrm{Ob}(\mathrm{C})$, an object $\mathrm{C}(A, B)$ in $\mathrm{V}$ called the "object of morphisms from $A$ to $B$ ";

- for all objects $A, B, C \in \mathrm{Ob}(\mathrm{C})$, a morphism $\bullet_{A, B, C}: \mathrm{C}(B, C) \otimes \mathrm{C}(A, B) \rightarrow \mathrm{C}(A, C)$ in $\mathrm{V}$ called the "composition";

- for every object $A \in \mathrm{Ob}(\mathrm{C})$, a morphism $\mathbf{1}_{A}: I \rightarrow \mathrm{C}(A, A)$ in $\mathrm{V}$ called the "identity on $A "$.

This data must satisfy the associativity and unit axioms, which are expressed by commutativity of the diagrams
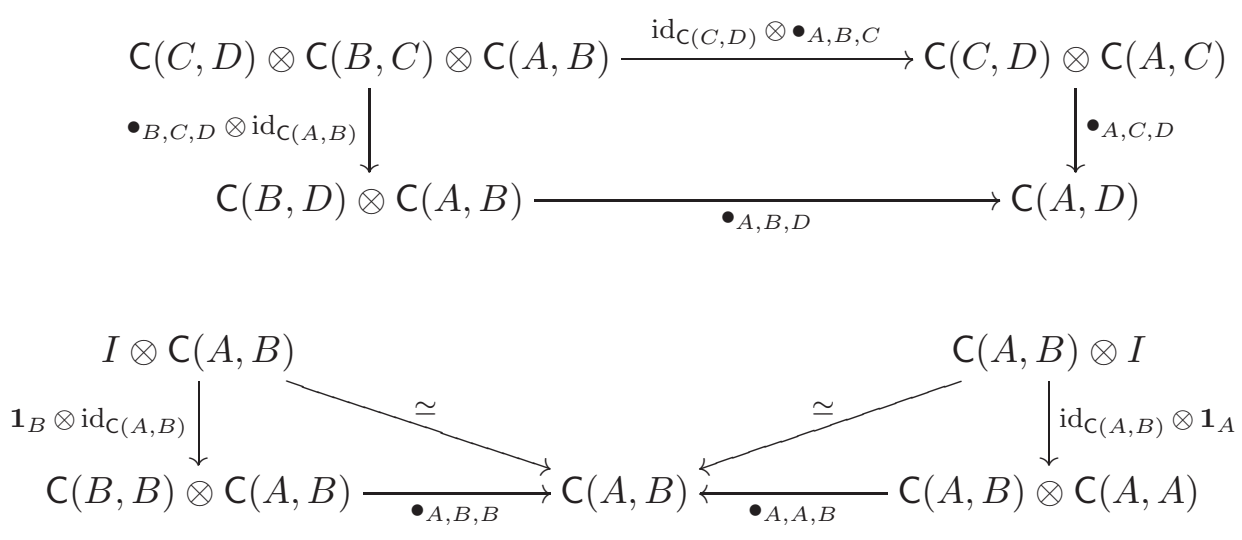

in the category $\mathrm{V}$, for all objects $A, B, C, D \in \mathrm{Ob}(\mathrm{C})$.

Remark A.2. For ease of notation, we shall always drop the labels on the composition and identity, i.e. we simply write $\bullet: \mathrm{C}(B, C) \otimes \mathrm{C}(A, B) \rightarrow \mathrm{C}(A, C)$ and $\mathbf{1}: I \rightarrow \mathrm{C}(A, A)$.

Definition A.3. Let $C$ and $D$ be two $V$-categories. A $V$-functor (or enriched functor) $\mathfrak{F}: C \rightarrow D$ is given by the following assignment:

- for every object $A \in \mathrm{Ob}(\mathrm{C})$, an object $\mathfrak{F}(A) \in \mathrm{Ob}(\mathrm{D})$; 
- for all objects $A, B \in \mathrm{Ob}(\mathrm{C})$, a morphism $\mathfrak{F}_{A, B}: \mathrm{C}(A, B) \rightarrow \mathrm{D}(\mathfrak{F}(A), \mathfrak{F}(B))$ in $\mathrm{V}$.

This assignment must be compatible with the composition and identity, which is expressed by commutativity of the diagrams
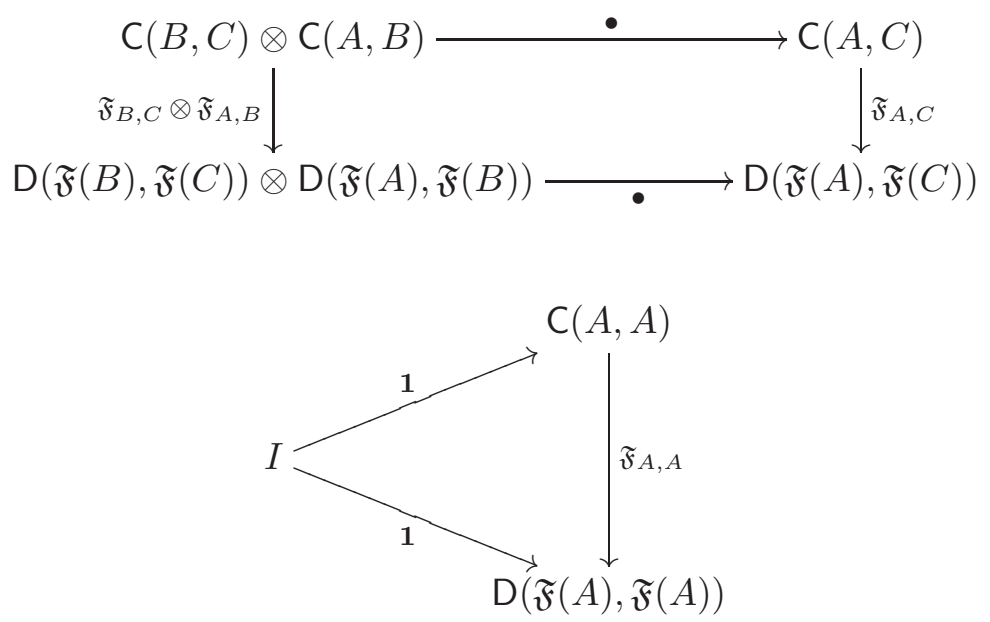

in the category $\mathrm{V}$, for all objects $A, B, C \in \mathrm{Ob}(\mathrm{C})$.

Remark A.4. Notice that $\mathrm{V}$-functors can be composed: Consider three $\mathrm{V}$-categories C, D, E and two $\mathrm{V}$-functors $\mathfrak{F}: \mathrm{C} \rightarrow \mathrm{D}$ and $\mathfrak{G}: \mathrm{D} \rightarrow \mathrm{E}$. We define the $\mathrm{V}$-functor $\mathfrak{G} \circ \mathfrak{F}: \mathrm{C} \rightarrow \mathrm{E}$ by the following assignment: To every object $A \in \mathrm{Ob}(\mathrm{C})$ we assign $(\mathfrak{G} \circ \mathfrak{F})(A):=\mathfrak{G}(\mathfrak{F}(A)) \in \operatorname{Ob}(\mathrm{E})$. To all objects $A, B \in \mathrm{Ob}(\mathrm{C})$ we assign the $\mathrm{V}$-morphism

$$
(\mathfrak{G} \circ \mathfrak{F})_{A, B}:=\mathfrak{G}_{\mathfrak{F}(A), \mathfrak{F}(B)} \circ \mathfrak{F}_{A, B}: \mathrm{C}(A, B) \longrightarrow \mathrm{E}(\mathfrak{G}(\mathfrak{F}(A)), \mathfrak{G}(\mathfrak{F}(B))),
$$

where $\circ$ denotes the composition of morphisms in $V$. It is easy to check that $\mathfrak{G} \circ \mathfrak{F}: C \rightarrow E$ is a V-functor.

Definition A.5. Let $\mathrm{C}$ and $\mathrm{D}$ be two $\mathrm{V}$-categories and $\mathfrak{F}, \mathfrak{G}: \mathrm{C} \rightarrow \mathrm{D}$ two parallel $\mathrm{V}$-functors. A $\mathrm{V}$-natural transformation (or enriched natural transformation) $\eta: \mathfrak{F} \Rightarrow \mathfrak{G}$ is given by assigning to every object $A \in \mathrm{Ob}(\mathrm{C})$ a morphism $\eta_{A}: I \rightarrow \mathrm{D}(\mathfrak{F}(A), \mathfrak{G}(A))$ in $\mathrm{V}$, such that the diagram

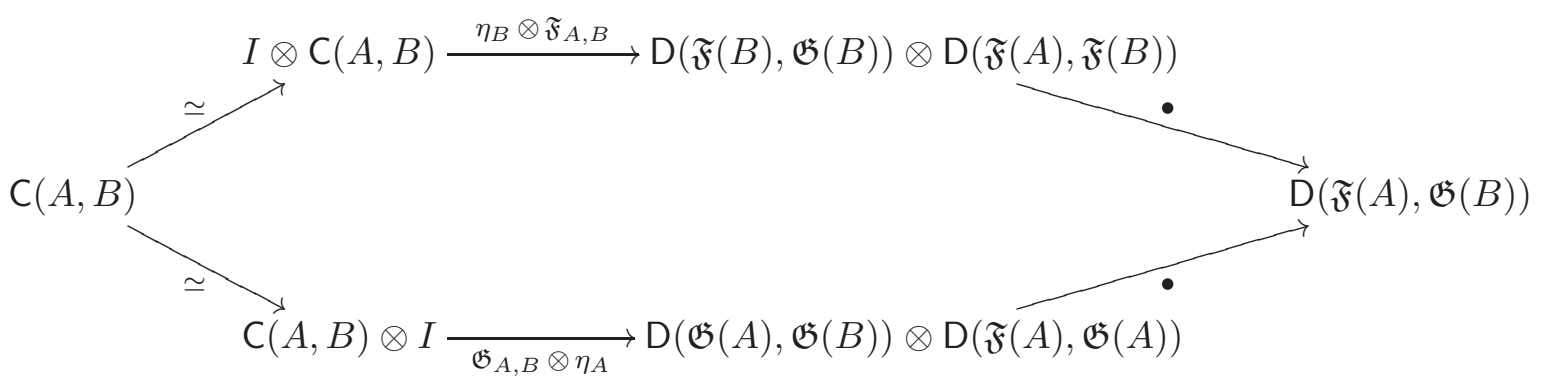

in the category $\mathrm{V}$ commutes, for all objects $A, B \in \mathrm{Ob}(\mathrm{C})$.

Remark A.6. Notice that for $\mathrm{V}$ being the monoidal category Set of sets, with monoidal bifunctor given by the Cartesian product and unit object given by any singleton set pt := $\{\star\}$, all definitions above reduce to the definitions in ordinary category theory. Hence, category theory enriched over Set is the same as ordinary category theory. 


\section{References}

[AL12] A. Alldridge and M. Laubinger, "Infinite-dimensional supermanifolds over arbitrary base fields," Forum Math. 24, 565-608 (2012) [arXiv:0910.5430 [math.DG]].

[BBHR91] C. Bartocci, U. Bruzzo and D. Hernández Ruipérez, "The geometry of supermanifolds," Mathematics and its Applications 71, Kluwer Academic Publishers Group, Dordrecht (1991).

[BDF09] R. Brunetti, M. Duetsch and K. Fredenhagen, "Perturbative Algebraic Quantum Field Theory and the Renormalization Groups," Adv. Theor. Math. Phys. 13, 1541 (2009) [arXiv:0901.2038 [math-ph]].

[BFV03] R. Brunetti, K. Fredenhagen and R. Verch, "The Generally covariant locality principle: A New paradigm for local quantum field theory," Commun. Math. Phys. 237, 31 (2003) [math-ph/0112041].

[BG06] D. Buchholz and H. Grundling, "Algebraic Supersymmetry: A Case study," Commun. Math. Phys. 272, 699 (2007) [math-ph/0604044].

[BG11] C. Bär and N. Ginoux, "Classical and quantum fields on Lorentzian manifolds," Springer Proc. Math. 17, 359 (2011) [arXiv:1104.1158 [math-ph]].

[BGP07] C. Bär, N. Ginoux and F. Pfäffle, "Wave equations on Lorenzian manifolds and quantization," Zürich, Switzerland: Eur. Math. Soc. (2007) [arXiv:0806.1036 [math.DG]].

[BK98] I. L. Buchbinder and S. M. Kuzenko, "Ideas and methods of supersymmetry and supergravity: Or a walk through superspace," Bristol, UK: IOP (1998).

[Bou89] N. Bourbaki, "Elements of Mathematics, Algebra I, Chapters 1-3," Springer-Verlag, New York (1989).

[BS00] F. Borceux and I. Stubbe, "Short introduction to enriched categories," Current research in operational quantum logic, Fundamental Theories of Physics 111, 167-194 (2000).

[CCF11] C. Carmeli, L. Caston and R. Fioresi, "Mathematical Foundations of Supersymmetry," EMS Series of Lectures in Mathematics (2011).

[CHKLX12] S. Carpi, R. Hillier, Y. Kawahigashi, R. Longo and F. Xu, "N=2 superconformal nets," arXiv:1207.2398 [math.OA].

[CKL08] S. Carpi, Y. Kawahigashi and R. Longo, "Structure and classification of superconformal nets," Annales Henri Poincare 9, 1069 (2008) [arXiv:0705.3609 [math-ph]].

[Del99] P. Deligne, "Notes on Spinors," in: P. Deligne et. al. (ed.) Quantum fields and strings: a course for mathematicians. Vol. 1, American Mathematical Society, Providence, RI, Institute for Advanced Study (IAS), Princeton, NJ (1999).

[DF99] P. Deligne and D. S. Freed, "Supersolutions," in: P. Deligne et. al. (ed.) Quantum fields and strings: a course for mathematicians. Vol. 1, American Mathematical Society, Providence, RI, Institute for Advanced Study (IAS), Princeton, NJ (1999).

[DM99] P. Deligne and J. W. Morgan, "Notes on Supersymmetry (following Joseph Bernstein)," in: P. Deligne et. al. (ed.) Quantum fields and strings: a course for mathematicians. Vol. 1, American Mathematical Society, Providence, RI, Institute for Advanced Study (IAS), Princeton, NJ (1999). 
[dMH13] P. de Medeiros and S. Hollands, "Superconformal quantum field theory in curved spacetime," Class. Quant. Grav. 30, 175015 (2013) [arXiv:1305.0499 [hep-th]].

[Fre99] D. S. Freed, "Five lectures on supersymmetry," American Mathematical Society, Providence, RI (1999).

[FV12] C. J. Fewster and R. Verch, "Dynamical locality of the free scalar field," Annales Henri Poincare 13, 1675 (2012) [arXiv:1109.6732 [math-ph]].

[GSR79] M. T. Grisaru, W. Siegel and M. Rocek, "Improved Methods for Supergraphs," Nucl. Phys. B 159, 429 (1979).

[Han14] F. Hanisch, "A Supermanifold structure on Spaces of Morphisms between Supermanifolds," arXiv:1406.7484 [math.DG].

[HS13] T. P. Hack and A. Schenkel, "Linear bosonic and fermionic quantum gauge theories on curved spacetimes," Gen. Rel. Grav. 45, 877 (2013) [arXiv:1205.3484 [math-ph]].

[HW01] S. Hollands and R. M. Wald, "Local Wick polynomials and time ordered products of quantum fields in curved space-time," Commun. Math. Phys. 223, 289 (2001) [grqc/0103074].

[HW02] S. Hollands and R. M. Wald, "Existence of local covariant time ordered products of quantum fields in curved space-time," Commun. Math. Phys. 231, 309 (2002) [grqc/0111108].

[Kel82] G. M. Kelly, "Basic concepts of enriched category theory," London Mathematical Society Lecture Note Series 64, Cambridge University Press, Cambridge-New York (1982). Reprint in Repr. Theory Appl. Categ. No. 10 (2005).

[Mol84] V. Molotkov, "Infinite-dimensional $\mathbb{Z}_{2}^{k}$-supermanifolds," ICTP preprints, IC/84/183 (1984). Scanned version available at http://ccdb4fs.kek.jp/cgi-bin/img/allpdf?198506284

[Sac08] C. Sachse, "A Categorical Formulation of Superalgebra and Supergeometry," arXiv:0802.4067 [math.AG].

[Sac09] C. Sachse, "Global Analytic Approach to Super Teichmuller Spaces," arXiv:0902.3289 [math.AG].

[Sei93] N. Seiberg, "Naturalness versus supersymmetric nonrenormalization theorems," Phys. Lett. B 318, 469 (1993) [hep-ph/9309335].

[Sha97] R. W. Sharpe, "Differential Geometry: Cartan's Generalization of Klein's Erlangen Program," Springer, New York (1997).

[Shv84] A. S. Shvarts, "On The Definition Of Superspace," Theor. Math. Phys. 60, 657 (1984) [Teor. Mat. Fiz. 60, 37 (1984)].

[ST11] S. Stolz and P. Teichner, "Supersymmetric field theories and generalized cohomology," Mathematical Foundations of Quantum Field Theory and Perturbative String Theory, Proceedings of Symposia in Pure Mathematics, Vol. 83, 279-340, A.M.S. (2011).

[SW11] C. Sachse and C. Wockel, "The diffeomorphism supergroup of a finite-dimensional supermanifold," Adv. Theor. Math. Phys. 15, 285-323 (2011) [arXiv:0904.2726 [math.DG]].

[Wal84] R. M. Wald, "General Relativity," Chicago, USA: Univ. Pr. (1984). 
[WB92] J. Wess and J. Bagger, "Supersymmetry and supergravity," Princeton, USA: Univ. Pr. (1992).

[WZ77] J. Wess and B. Zumino, "Superspace Formulation of Supergravity," Phys. Lett. B 66, 361 (1977). 